\title{
Femtosekunden-Spektroskopie zur Protontransfer-Dynamik im elektronisch angeregten Zustand von 1.8-Dihydroxy-9.10-Anthrachinon
}

\author{
Dissertation \\ zur Erlangung des Doktorgrades \\ der Mathematisch-Naturwissenschaftlichen Fakultäten \\ der Georg-August-Universität zu Göttingen
}

vorgelegt von

Nicole Breidenassel, geb. Hartmann

aus Hildesheim

Göttingen 2003 
D 7

Referent: Prof.J.Troe

Korreferent: Prof.M.Buback

Tag der mündlichen Prüfung: 3.7.2003 
Femtosekunden-Spektroskopie zur Protontransfer-Dynamik im elektronisch angeregten Zustand von 1.8-Dihydroxy-9.10-Anthrachinon

In der vorliegenden Arbeit werden Untersuchungen zum strahlungslosen Relaxationsverhalten von 1.8-Dihydroxy-9.10-Anthrachinon (1.8-DHAQ) in Zusammenhang mit dem intramolekularen Protontransfer im ersten elektronisch angeregten Zustand vorgestellt. Hierzu wurden Pump-Probe-Experimente in Benzylalkohol, sowie Fluoreszenz-Aufkonvertierungs-Experimente in den drei verschiedenen Lösungsmitteln Benzylalkohol, Propylencarbonat und Tetrachlorkohlenstoff durchgeführt. Überdies wurde der Einfluss der Deuterierung der Hydroxylgruppen von 1.8-DHAQ in Tetrachlorkohlenstoff untersucht. Bei der Auswertung der Messdaten zeigt sich, dass der Protontransfer im angeregten Zustand von 1.8DHAQ weder durch eine separate Verschiebung des Protons innerhalb des quasiaromatischen Ringes in seiner Gesamtheit beschrieben werden kann, noch durch eine reine Deformation des Molekülgerüstes. Die Umstrukturierung im angeregten Zustand muss vielmehr mehrere Schwingungsfreiheitsgrade des Moleküls umfassen. Die Ergebnisse der Untersuchungen werden daher auf der Grundlage eines Lippincott-Schröder-Doppelminimum-Potentials entlang der Protontransferkoordinate interpretiert, wobei eine Kopplung der $(\mathrm{OH})$-Schwingungsmode an weitere Schwingungsfreiheitsgrade des Moleküls berücksichtigt wird.

\footnotetext{
Proton-transfer-dynamics of the electronically excited 1.8-dihydroxy9.10-anthraquinone studied by femtosecond spectroscopy

This thesis investigates the connection between the non-radiative relaxation dynamics of 1.8-dihydroxy-9.10-anthraquinone (1.8-DHAQ) and the intramolecular proton-transfer in the first electronically excited state. For this purpose pumpprobe-measurements in benzyl alcohol as well as fluorescence-up-conversion measurements in benzyl alcohol, propylenecarbonate and tetrachloromethane were carried out. In addition the influence of the deuteration of the hydroxylgroups of 1.8-DHAQ in tetrachloromethane was tested. Analysis of the data shows, that the proton-transfer of 1.8-DHAQ in the first excited state can neither be described by a separate shift of the proton in the quasi-aromatic ring nor merely by a deformation of the molecular skeleton. The rearrangement of the molecular structure in the excited state has to comprise several degrees of vibrational freedom of the molecule. Therefore the interpretation of the results of the measurements have to be based on a Lippincott-Schroeder double-minimum potential along the protontransfer coordinate taking account of the coupling between the $(\mathrm{OH})$-vibrational mode and other low frequency modes of the molecule.
} 



\section{Inhaltsverzeichnis}

1 Einleitung 1

2 Stand der Forschung $\quad 3$

2.1 Das Molekül 1.8-DHAQ . . . . . . . . . . . . . . . . . . . 3

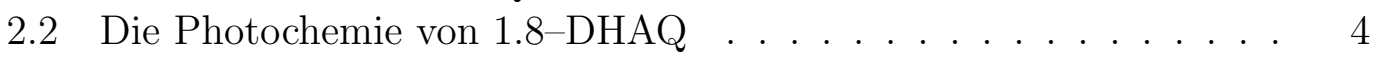

2.2.1 Ergebnisse frequenzaufgelöster Messungen . . . . . . . . 4

2.2.2 Ergebnisse zeitaufgelöster Messungen . . . . . . . . . . . . 8

2.2.3 Theoretische Grundlagen des Protontransfers . . . . . . . . 11

$\begin{array}{llr}3 & \text { Experimente } & 13\end{array}$

3.1 Lasersystem . . . . . . . . . . . . . . . . . . . . . . . . 13

3.2 Fluoreszenz-Aufkonvertierungs-Experiment . . . . . . . . . . 15

3.2 .1 Prinzip . . . . . . . . . . . . . . . . . . . . . . 15

3.2.2 Theorie der Summenfrequenzerzeugung . . . . . . . . . . . 16

3.2.3 Experimenteller Aufbau . . . . . . . . . . . . . . . . 21

3.2.4 Parameter des Messvorgangs . . . . . . . . . . . . . . 23

3.3 Pump-Probe-Experiment . . . . . . . . . . . . . . . 24

3.3.1 Prinzip . . . . . . . . . . . . . . . . . . . . 24

3.3.2 Experimenteller Aufbau . . . . . . . . . . . . . . . . 24

3.3.3 Parameter des Messvorgangs . . . . . . . . . . . . . . 26

3.4 Probenpräparation und Probenhalterung . . . . . . . . . . . 27

3.4.1 Probenlösungen . . . . . . . . . . . . . . . . . . . 27

3.4.2 Deuterierung . . . . . . . . . . . . . . 27

3.4.3 Frei fließender Probenlösungsstrahl . . . . . . . . . . . . . . 28

3.4.4 Geschlossene Rotationszelle . . . . . . . . . . . . . . 28

3.5 Messung stationärer Spektren . . . . . . . . . . . . . . 28

4 Ergebnisse und Datenanalyse $\quad 29$

4.1 1.8-DHAQ in Benzylalkohol . . . . . . . . . . . . . . . . . . 29

4.1.1 Stationäre Spektren . . . . . . . . . . . . . . . . . . . 29

4.1.2 Pump-Probe-Transienten . . . . . . . . . . . . . . . . 30

4.1.3 Fluoreszenzdynamik . . . . . . . . . . . . . . . . 35

$4.2 \quad 1.8$-DHAQ in Propylencarbonat . . . . . . . . . . . . . . . 39 
4.2 .1 Stationäre Spektren . . . . . . . . . . . . . . . . . . . . . . 39

4.2 .2 Fluoreszenzdynamik . . . . . . . . . . . . . . . . . . . . . 40

4.3 1.8-DHAQ in Tetrachlorkohlenstoff . . . . . . . . . . . . . . 44

4.3.1 Einfluss der Deuterierung auf die stationären Spektren . . 44

4.3.2 Einfluss der Deuterierung auf die Fluoreszenzdynamik . . . 45

5 Diskussion 49

5.1 Ursprung der Emission . . . . . . . . . . . . . . . . . . 51

5.2 Instantane Stokesverschiebung . . . . . . . . . . . . . . . . 53

5.3 Zuordnung der Zeitkonstanten . . . . . . . . . . . . . . . . 54

5.4 Lösungsmittelabhängigkeit . . . . . . . . . . . . . . . . 55

5.5 Einfluss der Deuterierung. . . . . . . . . . . . . . . 56

5.6 Intensitätsmodulationen im Pump-Probe-Signal . . . . . . . . 57

6 Zusammenfassung und Ausblick 61

$\begin{array}{ll}\text { Anhang } & 62\end{array}$

A Tabellen $\quad 63$

$\begin{array}{ll}\text { B Lippincott-Schröder-Potential } & 67\end{array}$

$\begin{array}{ll}\text { Literaturverzeichnis } & 68\end{array}$ 


\section{Kapitel 1}

\section{Einleitung}

Der Transfer eines Protons oder Wasserstoffatoms kann als eine der einfachsten chemischen Reaktionen betrachtet werden und ist ein grundlegender Baustein vieler chemischer und biochemischer Reaktionen von Photostabilisatoren in synthetischen Polymeren bis hin zu Enzymen, die viele Reaktionen in biologischen Systemen großer Komplexität katalysieren [1-6]. Da große spektrale Änderungen mit dem Transfer einhergehen, könnte man diesen möglicherweise auch als Grundlage für optische Speicher oder Schalter auf molekularer Ebene nutzen oder für den Bau eines Protontransferlasers. Seit den wegbereitenden Arbeiten von Weller [7] wird der Begriff des Proton-Transfers oft synonym mit dem des H-Atom-Transfers verwendet. Dank der Entwicklung neuer experimenteller und theoretischer Methoden konnte in den letzten Jahren ein immer detaillierteres Bild der ablaufenden Prozesse entwickelt werden [8-11] und noch immer ist insbesondere der ultraschnelle Protontransfer im angeregten Zustand ein ergiebiges Thema aktuellen Forschungsinteresses [12-26].

Intramolekulare Protontransfer-Reaktionen im angeregten Zustand (ESIPT $\left.{ }^{1}\right)$ können durch eine optische Anregung der thermodynamisch stabilen Molekülkonfiguration des Grundzustandes (sog. 'Normalform') eingeleitet werden. Die Art der darauf folgende Umstrukturierung im elektronisch angeregten Zustand ist abhängig vom betrachteten Molekül. Entweder beinhaltet sie eine separate Bewegung des Protons von einer Molekülgruppe $\left(-\mathrm{OH},-\mathrm{NH}_{2}\right)$ zu einer anderen $(-\mathrm{C}=\mathrm{O}$, $-\mathrm{N}=$ ) und resultiert in ein zwitterionisches Tautomer, oder es findet der Transfer eines ganzen Wasserstoffatoms statt, der neben der Umverteilung der elektronischen Ladungsverteilung wahrscheinlich auch eine globale Umordnung der Kerne des Molekülgerüstes umfasst und ein neutrales Tautomer zur Folge hat. Das resultierende Tautomer stellt die energetisch favorisierte Molekülkonfiguration im elektronisch angeregten Zustand dar und ist verantwortlich für die starke Stokesverschiebung der Fluoreszenz eines ESIPT aufweisenden Moleküls. Der Um-

\footnotetext{
${ }^{1} \mathrm{ESIPT}=$ Excited State Intramolecular Proton Transfer
} 
strukturierungsprozess im elektronisch angeregten Zustand kann je nach Molekül eine Barriere besitzen und das Tunneln eines Protons in einem DoppelminimumPotential beinhalten, oder einer barrierelosen Relaxation innerhalb eines deformierten Einfachminimum-Potentials entsprechen. Dies erklärt auch die große Bandbreite der Zeitskalen von ps bis in den fs-Bereich, die für ESIPT-Reaktionen beobachtet werden.

Die Messung der stationären Spektren solcher Moleküle liefert Informationen über die Schwingungsmoden des Moleküls und lässt prinzipielle Rückschlüsse zu auf den Potentialverlauf im Grundzustand und im elektronisch angeregten Zustand. Auf der Grundlage der Potentiale lassen sich dann indirekt über Quantendynamiksimulationen gewonnene Aussagen über die zeitliche Entwicklung des Systems machen. In Lösungen wird die Stokesverschiebung der Emission jedoch auch durch die Solvatation des Moleküls und die Kopplung der Reaktionskoordinate an Moden des Lösungsmittels beeinflusst [27], was eine verlässliche Konstruktion der Potentiale verhindert. Mit Hilfe von zeitaufgelösten Messungen im fs-Bereich ist es nun möglich, die Umwandlung des Moleküls direkt zu untersuchen und über die experimentell beobachtete Dynamik des Prozesses Rückschlüsse auf die Eigenschaften der Potentialverläufe zu ziehen [28, 29].

Zeitauflösungen im fs-Bereich lassen sich mit experimentellen Techniken erzielen, die Laserpulse als optische Fenster zur Beobachtung von Moleküldynamiken nutzen. Eine Zeitauflösung in der Größenordnung der Laserpulsdauer lässt sich mit Hilfe von Pump-Probe-Transmissions-Messungen erreichen. Allerdings fällt in solchen Transmissions-Experimenten die Trennung von stimulierter Emission und transienter Absorption oft schwer, was die Interpretation der Beobachtungen erschwert oder verhindert. Eine weitere Methodik ist die der zuerst von Mahr und Hirsch genutzten Fluoreszenz-Aufkonvertierung [30]. In einem FluoreszenzAufkonvertierungs-Experiment wird ausschließlich die Emission des fluoreszierenden Molekülzustandes betrachtet. Leider muss dafür eine etwas schlechtere Zeitauflösung in Kauf genommen werden.

Ein bereits durch stationäre Messungen in der Gasphase, der Matrix bei tiefen Temperaturen und der flüssigen Phase untersuchtes Modellsystem für den ESIPT ist 1.8-Dihydroxy-9.10-Anthrachinon (1.8-DHAQ) [31-38]. Im Rahmen der vorliegenden Arbeit werden fs-zeitaufgelöste Pump-Probe- sowie FluoreszenzAufkonvertierungs-Messungen an 1.8-DHAQ in verschiedenen polaren und nichtpolaren Lösungsmitteln und an dem deuterierten Derivat von 1.8-DHAQ durchgeführt, um weitergehende Einblicke in die mit dem ESIPT-Prozess einhergehenden molekularen Umstrukturierungsprozesse im angeregten Zustand zu gewinnen. 


\section{Kapitel 2}

\section{Stand der Forschung}

\subsection{Das Molekül 1.8-DHAQ}

Dihydroxy-Derivate von 9.10-Anthrachinon sind von Interesse als mögliche Farbstoffe für Flüssig-Kristall-Anzeigen [39], sowie als Ausgangsmoleküle für biologisch aktive Substanzen, wie etwa Anthracyclin-Antitumormedikamente [40].

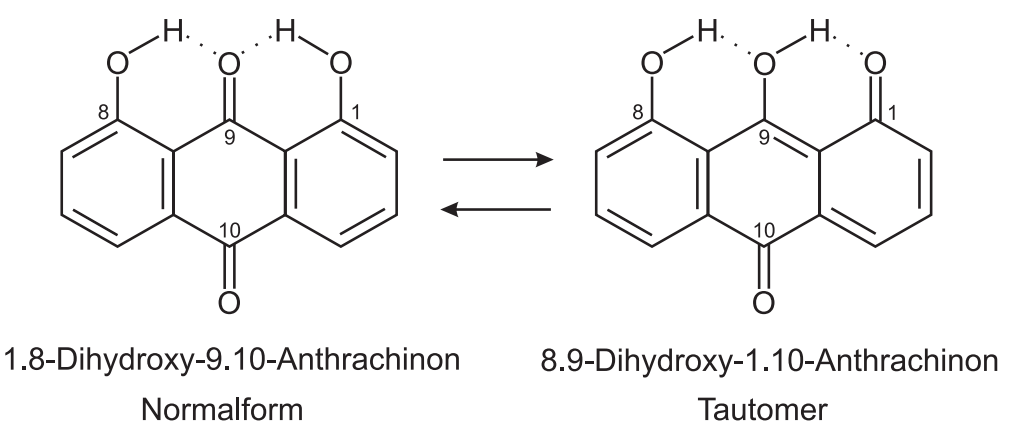

Abbildung 2.1: Strukturformel von 1.8-Dihydroxy-9.10-Anthrachinon (1.8-DHAQ), und seinem Tautomer 8.9-Dihydroxy-1.10-Anthrachinon. Die Punkte kennzeichnen Wasserstoffbrückenbindungen.

Unter den verschiedenen Systemen, an denen intramolekularer Protontransfer im angeregten Zustand untersucht wird, bietet sich die Gruppe der Anthrachinone besonders an, da eine leicht herzustellende Vielzahl von -OH und $-\mathrm{NH}_{2}$ Derivaten mit unterschiedlichen spektroskopischen Eigenschaften existiert. Unter den Dihydroxy-Isomeren von 9.10-Anthrachinon (DHAQ's) gibt es beispielsweise bei 1.4-DHAQ keinen Hinweis auf einen Protontransfer im angeregten Zustand, während die bei 1.5-DHAQ beobachtete duale Absorption und duale Fluoreszenz, sowie der beobachtete Einfluss der Deuterierung der OH-Gruppe auf das Spektrum eindeutig für die Existenz eines Protontransfer im angeregten Zustand spricht $[8,35,38]$. Auch bei 1.8-DHAQ ist eine duale Anregung, sowie eine dua- 
le Emission zu finden, wenn auch die Dualität im Absorptionsspektrum weniger ausgeprägt ist als bei 1.5-DHAQ. Eben deshalb ist es von Interesse die spektroskopischen Eigenschaften von 1.8-DHAQ als Modellsystem für ein "intermediäres" ESIPT-System genauer zu untersuchen. Gerade bei solch einem System wäre es aufschlussreich den Einfluss der Molekülumgebung auf den intramolekularen Protontransfer zu studieren. Ein weiterer Aspekt der die Untersuchung von 1.8-DHAQ interessant macht, liegt in der Molekülsymmetrie im elektronischen Grundzustand. Es existieren zwei äquivalente Wasserstoffbrücken zu einem gemeinsamen Protonenakzeptor (vgl. Abb. 2.1). Ein doppelter Protontransfer erscheint, da das $\mathrm{O}_{9}$-Sauerstoffatom in diesem Fall drei Bindungen eingehen müsste, unwahrscheinlich. Dies wirft die Frage nach den Ursachen des Symmetriebruchs auf, der für den Transfer eines der beiden Protonen erforderlich ist.

\subsection{Die Photochemie von 1.8-DHAQ}

Wie bereits beschrieben ist 1.8-DHAQ ein interessantes Modellmolekül für intramolekularen Protontransfer im angeregten Zustand (ESIPT) ${ }^{1}$ (vgl. Abb. 2.1). Im folgenden soll ein Überblick über die bereits von anderen Forschungsgruppen an 1.8-DHAQ durchgeführten, frequenzaufgelösten und zeitaufgelösten Messungen und deren Interpretation der Daten gegeben werden.

\subsubsection{Ergebnisse frequenzaufgelöster Messungen}

Eine Reihe von frequenzaufgelösten Untersuchungen an 1.8-DHAQ wurden seit Mitte der 90er Jahre von Smulevich et al. durchgeführt. Hierzu zählen die Messungen von resonanten Raman-Spektren und von Anregungs- und EmissionsSpektren in n-Alkan-Shpol'skii-Matrizen bei 8-10 K [32-34], und in $\mathrm{CCl}_{4}$-Lösung bei Raumtemperatur [35], sowie an kristallinen Formen von 1.8-DHAQ gemessene Infrarot-Spektren [31]. Von Gillispie et al. wurden zu dieser Zeit auch Messungen von Anregungs- und Emissions-Spektren in der Gasphase [38] durchgeführt, sowie weitere Untersuchungen in n-Oktan Shpol'skii-Matrizen bei tiefen Temperaturen. Beide Gruppen haben auch den Einfluss einer Deuterierung der OH-Gruppen untersucht.

Die von Smulevich et al. und Gillispie et al. in der Shpol'skii-Matrix, der Gasphase, und in Lösung beobachteten Emissionsspektren sind qualitativ sehr ähnlich, und lassen sich in zwei klar zu trennende Spektralbereiche unterteilen. Gleiches gilt für die in der Shpol'skii-Matrix und der Gasphase gemessenen Anregungsspektren. Die Emission bei kürzeren Wellenlängen weist schwächere Intensitäten

\footnotetext{
${ }^{1} \mathrm{ESIPT}=$ Excited State Proton Transfer
} 
auf und besitzt bei tiefen Temperaturen eine gut definierte Schwingungsstruktur. Die Emission bei längeren Wellenlängen dagegen hat eine wesentlich höhere Intensität und zeigt unstrukturierte Banden. Bei der Anregung verhält es sich genau umgekehrt.

Diese beobachtete Dualität ${ }^{2}$ liefert einen deutlichen Hinweis für das Auftreten eines Protontransfers im angeregten Zustand nach erfolgter elektronischer Anregung. Ein solcher Protontransfer lässt sich auf der Grundlage eines stark anharmonischen Doppelminimum-Potentials entlang der Protontransfer-Koordinate beschreiben [11]. Hierbei ist die Symmetrie im angeregten Zustand umgekehrt zu der im Grundzustand anzunehmen. Zur besseren Veranschaulichung ist ein solches Potential in Abbildung 2.2 schematisch dargestellt. Aufgetragen ist die Gesamtenergie des Moleküls in Abhängigkeit von der Protontransfer-Koordinate $(\mathrm{r}(\mathrm{O}-\mathrm{H})$ im Fall von 1.8-DHAQ). Das energetisch tiefer liegende Minimum im Grundzustand entspricht der Normalform des Moleküls, hier mit $\mathrm{S}_{0}$ bezeichnet. Das energetisch höher liegende zweite Minimum entspricht der protontransferierten Form des Moleküls, im folgendem $\mathrm{S}_{0}^{\prime}$ genannt. Entsprechend ist das energetisch höher liegende Minimum des ersten angeregten Zustandes der lokal angeregten Normalform $S_{1}$ zuzuordnen, und das tiefere Minimum des ersten angeregten Zustandes der protontransferierten Form $\mathrm{S}_{1}^{\prime}$.

Sowohl Gillispie et al. als auch Smulevich et al. verwendeten für die genauere Analyse ihrer Daten ein Doppelminimum-Potential entlang der $\mathrm{r}(\mathrm{O}-\mathrm{H})$-Abstandskoordinate $^{3}$, das Lippincott und Schroeder Mitte der 60er Jahre zur Beschreibung linearer und gewinkelter Molekülsysteme mit Wasserstoffbrückenbindungen der Art $\mathrm{R}_{1} \mathrm{O}-\mathrm{H} \cdots \mathrm{OR}_{2}$ vorgeschlagen hatten (vgl. Anhang $\mathrm{B}$ ). In diesem Potential entsprechen die beiden Minima den beiden resonanten Formen $\mathrm{R}_{1} \mathrm{O}-\mathrm{H} \cdots \mathrm{OR}_{2}$ und $\mathrm{R}_{1} \mathrm{O}^{-} \cdots \mathrm{H}-\mathrm{O}^{+} \mathrm{R}_{2}$. Lippincott und Schroeder waren mit dem von ihnen vorgeschlagenen Potential in der Lage die Streckfrequenz $\nu(\mathrm{O}-\mathrm{H})$, den Abstand $\mathrm{r}(\mathrm{O}-\mathrm{H})$ und die Bindungsenergie der Wasserstoffbrücke in Abhängigkeit vom Abstand $\mathrm{R}(\mathrm{O} \cdots \mathrm{O})$ in guter Übereinstimmung mit experimentell gefundenen Werten zu berechnen $[42,43]$. Anfang der 90er Jahre führten Saitoh et al. auf der Grundlage von Lippincott-Schroeder-Potentialen (LS-Potentialen) zweidimensionale Schwingungsanalysen an $\mathrm{R}_{1} \mathrm{O}-\mathrm{H} \ldots \mathrm{OR}_{2}$ Wasserstoffbrückensystemen durch [44]. Sie zeigten außerdem, dass sich mit Hilfe des LS-Potentials auch der Deu-

\footnotetext{
${ }^{2}$ Von der Dualität eines Spektrums wird im folgenden immer dann gesprochen, wenn sich das Spektrum aus zwei klar zu trennenden Bereichen zusammensetzt. Dies ist zum Beispiel der Fall beim Auftreten eines zweiten Maximums oder einer ausgeprägten Schulter neben dem absoluten Maximum des Spektrums.

${ }^{3} \mathrm{Ab}$ initio Berechnungen von 1.8-DHAQ im Grundzustand wurden 2001 von Rodríguez et al. durchgeführt [41]. Hierbei ergab sich nur ein einzelnes Minimum im stark deformierten Potential entlang der r(O-H)-Koordinate. Es ist jedoch anzumerken, dass das Ergebnis von Ab initio Berechnungen entscheidend von der Wahl der eingesetzten Näherungsmethoden abhängt.
} 


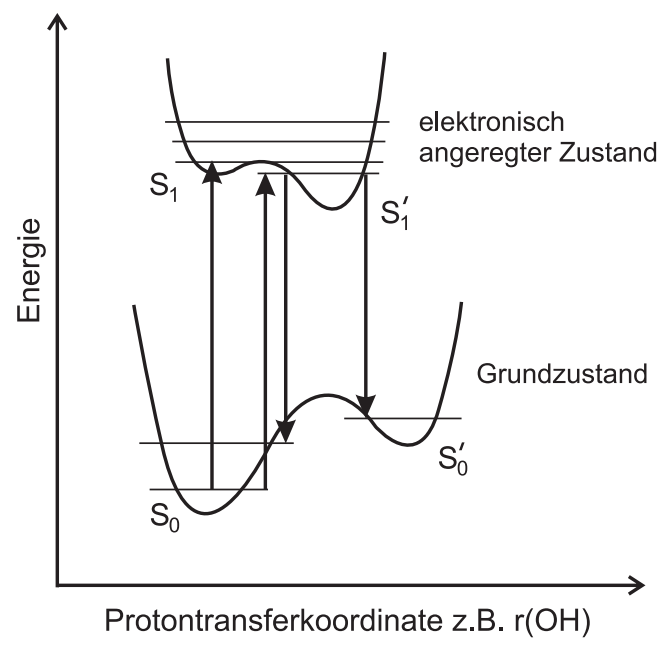

Abbildung 2.2: Protontransfer im angeregten Zustand (ESIPT) lässt sich auf der Grundlage stark anharmonischen Doppelminimum-Potentiale entlang der Protontransfer-Koordinate (hier $r(O-H)$ ) beschreiben. $S_{0}$ und $S_{1}$ entsprechen dem Grundzustand und ersten elektronisch angeregten Zustand der Normalform des Moleküls. $S_{0}$ ' und $S_{1}$ ' gehören zu den entsprechenden Zuständen der protontransferierten Form. Mit Pfeilen angedeutet sind mögliche vibronische Übergänge zwischen den Grundzuständen $S_{0}$ und $S_{0}$ ' und den angeregten Zuständen $S_{1}$ und $S_{1}$ '.

terium-Isotopen-Effekt gut beschreiben lässt, und sowohl Proton-Tunnelprozesse als auch eine Delokalisierung der Wellenfunktion des Protons über die Barriere hinaus in dem Potentialmodell beinhaltet sind. Sowohl die Berechnungen von Lippincott und Schroeder als auch die von Saitoh et al. bezogen sich auf den elektronischen Grundzustand intermolekularer Wasserstoffbrückensysteme in der kristallinen Phase.

Smulevich et al. wendeten das LS-Potential nun auch auf intramolekulare Wasserstoffbrückensysteme in Lösungen und den ersten elektronisch angeregten Zustand an. Sie ermittelten das LS-Potential für den Grundzustand von 1.8-DHAQ, aus der Forderung, dass es die in der dualen Emission beobachteten Frequenzabstände am besten reproduziert. Die beobachtete Emission bei kürzeren Wellenlängen wurde hierbei dem Übergang $\mathrm{S}_{1}^{\prime} \longrightarrow \mathrm{S}_{0}$ zugeordnet, und die Emission bei längeren Wellenlängen dem Übergang $\mathrm{S}_{1}^{\prime} \longrightarrow \mathrm{S}_{0}^{\prime}$. Es sei erwähnt, dass der rein elektronische Übergang $\mathrm{S}_{1}^{\prime} \longrightarrow \mathrm{S}_{0}$ nur in der Matrix gefunden wird. In $\mathrm{CCl}_{4}$-Lösungen beobachtet man nur den Übergang von $\mathrm{S}_{1}^{\prime}$ in die erste $\nu(\mathrm{O}-\mathrm{H})$-Streckschwingungsmode des $\mathrm{S}_{0}$-Grundzustandes als Schulter an der kurzwelligen Seite des $\mathrm{S}_{1}^{\prime} \longrightarrow \mathrm{S}_{0}^{\prime}$ Übergangs. Das LS-Potential für den angeregten Zustand wurde entsprechend so gewählt, dass es die duale Absorption mit den Übergängen $S_{0} \longrightarrow S_{1}$ und $\mathrm{S}_{0} \longrightarrow \mathrm{S}_{1}^{\prime}$ optimal beschreibt. Die aus den beobachteten Frequenzabständen ab- 
geleiteten Energien der rein elektronischen Übergänge in $\mathrm{CCl}_{4}$-Lösung sind in Tabelle 2.1 aufgeführt.

\begin{tabular}{|lcc|}
\hline Übergang & $\begin{array}{c}\text { Energie } \\
{\left[\mathrm{cm}^{-1}\right]}\end{array}$ & $\begin{array}{c}\lambda \\
{[\mathrm{nm}]}\end{array}$ \\
\hline \hline $\mathrm{S}_{0} \longrightarrow \mathrm{S}_{1}+3 \nu_{O H}$ & 25700 & 390 \\
$\mathrm{~S}_{0} \longrightarrow \mathrm{S}_{1}$ & 21820 & 458 \\
$\mathrm{~S}_{0} \longrightarrow \mathrm{S}_{1}^{\prime}$ & 21200 & 472 \\
\hline $\mathrm{S}_{1} \longrightarrow \mathrm{S}_{0}$ & 21000 & 476 \\
$\mathrm{~S}_{1}^{\prime} \longrightarrow \mathrm{S}_{0}+\nu_{O H}$ & 17900 & 558 \\
$\mathrm{~S}_{1}^{\prime} \longrightarrow \mathrm{S}_{0}^{\prime}$ & 17070 & 586 \\
\hline
\end{tabular}

Tabelle 2.1: Elektronische und vibronische Übergänge für 1.8-DHAQ in $C C l_{4}$ nach Smulevich et al. [35]

Für den Grundzustand von 1.8-DHAQ wurde auf diese Weise ein $\mathrm{R}(\mathrm{O} \cdots \mathrm{O})$ Abstand von $\mathrm{R}_{0}(\mathrm{O} \cdots \mathrm{O}) \approx 2.62 \AA$ gefunden, und für den ersten angeregten Zustand $\mathrm{R}_{1}(\mathrm{O} \cdots \mathrm{O}) \approx 2.46 \AA$. Die mit den so ermittelten Potentialen durch Betrachtung der Franck-Condon-Faktoren berechneten relativen Intensitäten der Übergänge sind konsistent mit den experimentell beobachteten. Auch die bei deuteriertem 1.8DHAQ experimentell gefundene gegenüber dem nichtdeuterierten Molekül verringerte Wahrscheinlichkeit der Übergänge $S_{1}^{\prime} \longrightarrow S_{0}$ und $S_{0} \longrightarrow S_{1}^{\prime}$ lässt sich daraus ableiten.

Allgemein nimmt die Höhe der Barriere im LS-Potential entlang der r(O-H)Koordinate mit zunehmendem $\mathrm{R}(\mathrm{O} \cdots \mathrm{O})$ zu. Ein quantenmechanischer Zustand unterhalb der Barriere existiert jedoch erst dann, wenn die Höhe der klassischen Barriere größer ist als die Nullpunktsenergie des Zustandes. Berechnungen durch Smulevich et al. zeigen, dass für den Grundzustand von 1.8-DHAQ gebundene Zustände in beiden Minima existieren. Im angeregten Zustand dagegen reicht die Tiefe des $S_{1}^{\prime}$ Minimum gerade für ein knapp unterhalb der Barriere befindliches Nullpunktsniveau aus, während das etwa $600 \mathrm{~cm}^{-1}$ darüberliegende $\mathrm{S}_{1}$ Minimum $\mathrm{zu}$ flach ist, um einen gebundenen Zustand zu enthalten $\left(1000 \mathrm{~cm}^{-1}\right.$ Nullpunktsenergie, $475 \mathrm{~cm}^{-1}$ klassische Barriere). Es existiert somit keine quantenmechanische Barriere für den Protontransfer im angeregten Zustand von 1.8-DHAQ. Die Wellenfunktionen der angeregten vibronischen Zustände sind über beide Minima delokalisiert. Dies ist auch konsistent mit der von Smulevich et al. im Absorptionsspektrum beobachteten $\sim 1300 \mathrm{~cm}^{-1}-\nu(\mathrm{O}-\mathrm{H})$-Progression, die für ein näherungsweise harmonisches Potential im angeregten Zustand von 1.8-DHAQ spricht.

Gillispie et al. kamen trotz Kritik am Modell von Smulevich et al. durch Plausibilitätsbetrachtungen auch zu dem Resultat, dass wahrscheinlich kein gebundener 
Zustand im $\mathrm{S}_{1}$ Minimum existiert und der Potentialverlauf entlang der Protontransferkoordinate dem eines verzerrten Einzelminimum-Potentials entspricht.

Für die spätere Diskussion sei erwähnt, dass Smulevich et al. im Fluoreszenzspektrum und dem resonanten Raman-Spektrum der Matrix ein starkes Band bei (209-219) $\mathrm{cm}^{-1}$ beobachteten, das sie in ihren früheren Veröffentlichungen [32-34] einer Biegeschwingung in der Ebene des Molekülgerüstes im elektronischen Grundzustand zuordneten, die große Bewegungen der beiden O-H-Gruppen beinhaltet. Demgegenüber machten sie in ihrer letzten Veröffentlichung [35] für dieselbe Bande die $\delta(C-O)$-Biegeschwingung verantwortlich. Umgekehrt verhielt es sich bei der Zuordnung der von ihnen bei (302-324) $\mathrm{cm}^{-1}$ beobachteten Bande. Beiden Zuordnungen ist jedoch gemeinsam, dass durch sie der Abstand der Sauerstoffatome im durch Wasserstoffbrücken gebundenen C-O-H $\cdots \mathrm{O}=\mathrm{C}-\mathrm{Ring}$ moduliert wird. Auch die bei (338-344) $\mathrm{cm}^{-1}$ beobachtete Gerüstschwingung beinhaltet nach Smulevich et al. wahrscheinlich eine Bewegung der Sauerstoffatome. Als korrespondierende Schwingungen im ersten elektronisch angeregten Zustand wurden von Smulevich et al. die bei $242 \mathrm{~cm}^{-1}, 357 \mathrm{~cm}^{-1}$ und $397 \mathrm{~cm}^{-1}$ im Anregungsspektrum beobachteten Banden angesehen.

Gillispie et al. unterstützten die Interpretation der Schwingungen bei $209 \mathrm{~cm}^{-1}$ und bei $338 \mathrm{~cm}^{-1}$ als Gerüstschwingung, bestreiten aber die Existenz einer Fundamentalen bei $302 \mathrm{~cm}^{-1}$. Darüberhinaus betonten sie, dass das auch von ihnen bei $249 \mathrm{~cm}^{-1}$ im Anregungsspektrum beobachtete Band kein offensichtliches Gegenstück zur $209 \mathrm{~cm}^{-1}$ Fundamentalen darstellt.

\subsubsection{Ergebnisse zeitaufgelöster Messungen}

Erste zeitaufgelöste Messungen des Anregungs- und Fluoreszenzspektrums von 1.8-DHAQ in verschiedenen Lösungsmitteln (Cyclohexan, Benzen und Ethanol) wurden 1990 mit einer Zeitauflösung von etwa 100 ps von Mittal et al. durchgeführt [45]. Das stationäre Fluoreszenzspektrum zeigte mit zunehmender Polarisation des Lösungsmittels eine geringe Abnahme der Intensität bei kürzeren Wellenlängen gegenüber der Intensität bei längeren Wellenlängen. Das bei allen Wellenlängen gefundene monoexponentielle Abklingverhalten der Fluoreszenzintensität mit der Zeit blieb hingegen unverändert. Das Anregungsspektrum wies keine Abhängigkeit vom Lösungsmittel auf. Aufgrund dieser Beobachtung schlugen Mittal et al. vor, den emittierenden $\mathrm{S}_{1}^{\prime}$-Zustand nicht als wirkliches Tautomer $\mathrm{C}_{1 / 8^{-}} \mathrm{O} \cdots \mathrm{H}-\mathrm{O}=\mathrm{C}_{9}$ anzusehen, sondern als eine protontransferierte Form des Moleküls mit geladener Struktur $\mathrm{C}_{1 / 8^{-}} \mathrm{O}^{-} \cdots \mathrm{H}_{-} \mathrm{O}^{+}=\mathrm{C}_{9}$. Nach ihrer Interpretation werden die Ladungen in polaren Lösungsmitteln und Lösungsmitteln, die intermolekulare Wasserstoffbrücken ausbilden, stabilisiert so dass der Übergang in das Tautomer unterdrückt wird. 
Eine entgegengesetzte Beobachtung wurde 2001 von Neuwahl und Bussotti gemacht [21]. Sie fanden im stationären Fluoreszenzspektrum von 1.8-DHAQ in polarem Acetonitril wesentlich stärkere Intensitäten bei kürzeren Wellenlängen als in unpolarem Cyclohexan. Für ihre Interpretation gehen sie davon aus, dass sich der für die Fluoreszenz bei Raumtemperatur verantwortliche Zustand signifikant von dem bei tiefen Temperaturen unterscheidet. Während bei tiefen Temperaturen im stationären Fluoreszenzspektrum ausschließlich Übergänge von $S_{1}^{\prime}$ in den Grundzustand beobachtet werden, existieren bei Raumtemperatur Übergänge aus beiden koexistierenden Zuständen $\mathrm{S}_{1}$ und $\mathrm{S}_{1}^{\prime}$ (vgl. Abb. 2.3). Bei Raumtemperatur ist $\mathrm{k}_{B} \mathrm{~T}$ vergleichbar mit der Energiedifferenz zwischen den beiden Zuständen $\mathrm{S}_{1}$ und $\mathrm{S}_{1}^{\prime}$ im angeregten Zustand. Die Fluoreszenz bei kürzeren Wellenlängen ist hier also dem Übergang $S_{1} \longrightarrow S_{0}$ zuzuordnen und die Fluoreszenz bei längeren Wellenlängen dem Übergang $S_{1}^{\prime} \longrightarrow S_{0}^{\prime}$. Diese Argumentation ist auch konsistent mit dem von Smulevich et al. entwickelten Potentialmodell. Bei $\mathrm{T}=10 \mathrm{~K}$ war nur der energetisch tiefste Zustand im $\mathrm{S}_{1}^{\prime}$-Minimum besetzt, während bei $\mathrm{T}=298 \mathrm{~K}$ auch der darüberliegende bereits über beide Minima delokalisierte Zustand besetzt ist. Der von Neuwahl und Bussotti beobachtete Einfluss des Lösungsmittels entspricht folglich einer Stabilisierung der lokal angeregten Normalform $\mathrm{S}_{1}$ des Moleküls gegenüber dem Tautomer $\mathrm{S}_{1}^{\prime}$. Diese erklärten sie mit einem größeren Dipolmoment der lokal angeregte Normalform gegenüber der protontransferierten Form.
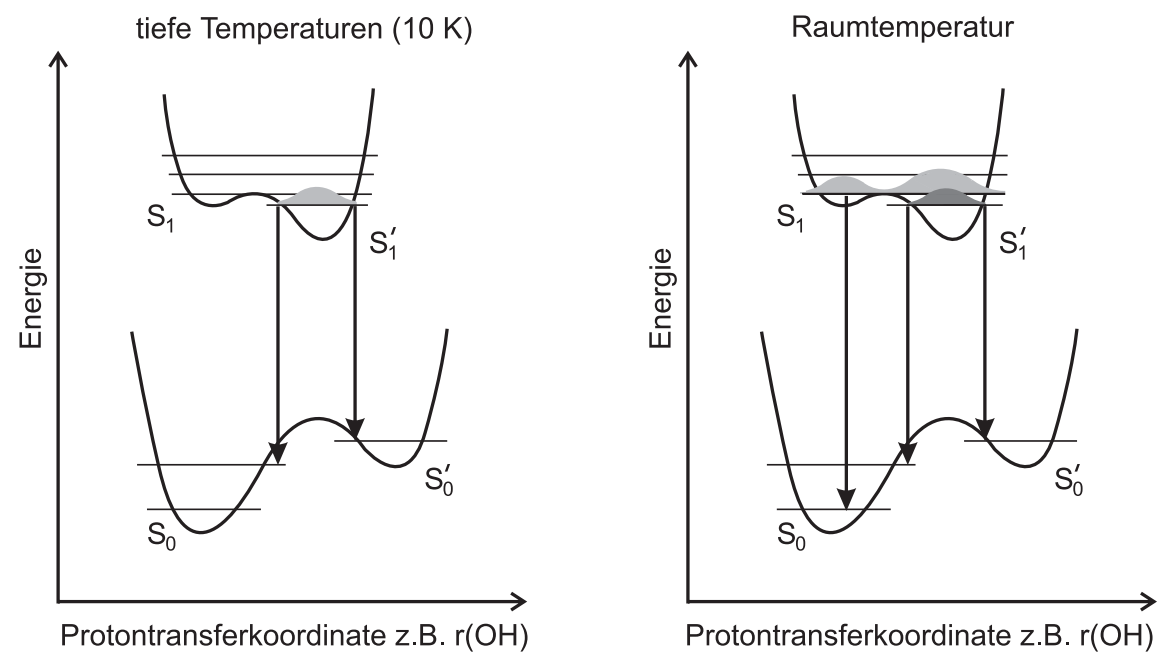

Abbildung 2.3: Als Ursprung der stationären Emission ist bei tiefen Temperaturen $S_{1}$ allein anzusehen, während bei Raumtemperatur Übergänge aus den beiden koexistierenden Zuständen $S_{1}$ und $S_{1}$ 'existieren.

Von Neuwahl und Bussotti wurde 2001 auch das transiente Absorptionsspektrum von 1.8-DHAQ in Acetonitrile mit einer Zeitaufösung von etwa 150 fs aufgenommen. Sie beobachteten, wie unsere Arbeitsgruppe Vöhringer et al. bereits ein 
Jahr zuvor in Benzylalkohol [46], transiente Absorption im Wellenlängenbereich bis etwa (560-570) nm und für längere Wellenlängen stimulierte Emission. Allerdings wurde mit der vorhandenen Zeitauflösung keine spektrale Entwicklung innerhalb der Lebensdauer des angeregten Zustandes beobachtet, wie sie von Vöhringer et al. bereits gemessen worden war. Die Messungen wurden für verschiedene Lösungsmittel und mit verschiedenen Anregungswellenlängen zwischen $400 \mathrm{~nm}$ und $450 \mathrm{~nm}$ wiederholt, mit analogem Ergebnis. Die Schlussfolgerung von Neuwahl und Bussotti war, dass der Protontransfer im angeregten Zustand von 1.8-DHAQ instantaner Art ist. Sie stimmten aber auch mit der von Vöhringer et al. veröffentlichten Interpretation überein, dass die ablaufenden Prozesse nicht als Protontransfer im klassischen Sinne anzusehen sind, sondern bei der optischen Anregung vielmehr ein über beide tautomere Formen delokalisierter angeregter Zustand erzeugt wird, mit einer vernachlässigbar kleinen Barriere zwischen den beiden Formen.

Untersuchungen zu zeitaufgelösten Fluoreszenzspektren bei Raumtemperatur wurden 2000 von Tahara et al. über 1.8-DHAQ in Hexan veröffentlicht [47]. Die Zeitauflösung betrug etwa $170 \mathrm{fs}$. Beobachtet wurde, dass beide Komponenten der dualen Fluoreszenz sofort nach der Anregung des Moleküls existieren. Innerhalb der ersten Pikosekunden ist ein Abklingen der Fluoreszenzintensität im kurzwelligen Bereich der Fluoreszenz, und ein Ansteigen im langwelligen Bereich zu beobachten. Nach etwa 5 ps zeigt die Fluoreszenz keine signifikanten spektralen Änderungen mehr, und fällt bei allen Wellenlängen monoton ab. Die Form des Spektrums ist dem stationären Fluoreszenzspektrum sehr ähnlich. Alle Kurven wurden mittels gefalteter triexponentieller Funktionen angepasst. Die Zeitkonstante auf der längsten Zeitskala lag für den gesamten beobachteten Wellenlängenbereich bei etwa $127 \pm 7$ ps, und entspricht der Lebensdauer des ersten elektronisch angeregten Zustands. Die zwei für den Bereich des Ansteigens bzw. Abklingens benötigten Zeitkonstanten hingegen hingen von der betrachteten Wellenlänge ab. Diese Beobachtung spricht dafür, dass sich die innerhalb der ersten Pikosekunden ablaufenden Prozesse nicht mit einfachen auf Ratengleichungen basierenden Modellen beschreiben lassen. Die beiden kleinsten Zeitkonstanten besitzen also keine klare physikalische Bedeutung.

Die Diskussion der Ergebnisse durch Tahara et al. basiert auf dem bereits beschriebenen Modell der Lippincott-Schroeder-Doppel-Minimum-Potentiale. Für das instantane Auftreten beider Bereiche der dualen Fluoreszenz machten Tahara et al. eine nach dem Anregungsprozess innerhalb von 50 fs stattfindende Delokalisierung des elektronisch angeregten Zustandes verantwortlich, und folgerten, dass ESIPT von 1.8-DHAQ in diesem Sinne als barriereloser Prozess angesehen werden kann, zumindest im Fall der Anregung mit einem Energieüberschuss von etwa $3000 \mathrm{~cm}^{-1}$ gegenüber dem rein elektronischen Übergang. Hier unterstützten sie die von Vöhringer et al. in einer vorherigen Veröffentlichung präsentierte 
Interpretation, dass die Intensität der kurzwelligen und der langwelligen Fluoreszenz als Maß für den Franck-Condon-Überlapp zwischen der delokalisierten Wellenfunktion im ersten elektronisch angeregten Zustand und der Wellenfunktion der beiden Tautomere im Grundzustand wiederspiegelt. Die normale und tautomere Form können jedoch nicht als zwei unterschiedliche chemische Spezies im angeregten Zustand angesehen werden. Die zusätzliche Verschiebung der Lage des Protons innerhalb von $\sim 2$ ps betrachteten sie als Folge der intramolekularen Schwingungsrelaxation aufgrund von Umverteilungsprozessen der Energie zwischen verschiedenen Schwingungsfreiheitsgraden des Moleküls, da Übertragung von Schwingungsenergien des gelösten Probenmoleküls auf die umliegenden Hexanmoleküle bis dato nur auf Zeitskalen von (10-20) ps beobachtet wurden.

\subsubsection{Theoretische Grundlagen des Protontransfers}

Nagaoka und Nagashima postulierten 1996, dass das bei Hydroxyanthrachinonen in Verbindung mit dem intramolekularen Protontransfer beobachtete spektroskopische Verhalten, durch Betrachtung der Knotenstruktur der Wellenfunktion im angeregten Zustand erklärt werden kann $[48]^{4}$.

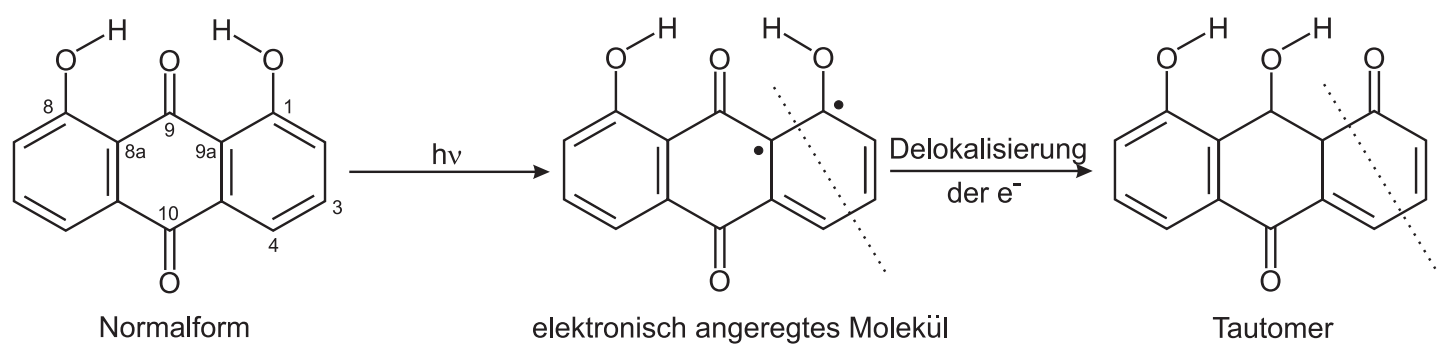

Abbildung 2.4: ESIPT kann durch Betrachtung des Knotenverlaufs der Wellenfunktion im angeregten Zustand erklärt werden. Die beiden Punkte kennzeichnen die beiden ungepaarten Elektronen, die gepunktete Linie die Knotenebene (nach [48]).

Im niedrigsten angeregten Singulettzustand mit $\pi \pi^{*}$ Charakter gibt es bei 1.8DHAQ aufgrund seiner Spiegelsymmetrie zwei Möglichkeiten für den Verlauf der Knotenebene der $\pi$-Elektron-Wellenfunktion. Die senkrecht zur Molekülebene stehende Knotenebene kann zwischen den $\mathrm{O}_{1}$ und $\mathrm{O}_{9}$ Atomen und den $\mathrm{C}_{3}$ und $\mathrm{C}_{4}$ Atomen verlaufen, oder zwischen den dazu spiegelbildlich sitzenden Atomen. An den Atomen $\mathrm{C}_{1}$ und $\mathrm{C}_{9 a}$ bzw. $\mathrm{C}_{8}$ und $\mathrm{C}_{8 a}$ befinden sich hierbei ungepaarte Elektronen. Da die beiden Knotenebenen weit voneinander weg liegen, können sie nicht miteinander koppeln, wie es zum Beispiel für 1.4-DHAQ, das keinen

\footnotetext{
${ }^{4}$ Hierbei nahmen sie an, dass ESIPT im Schwingungsgrundzustand stattfindet. Betrachtet wurde daher zunächst nur der elektronische Teil der Wellenfunktion, wobei betont wurde, dass die Übertragbarkeit auf den allgemeinen Fall dadurch nicht eingeschränkt wird.
} 
Protontransfer zeigt, der Fall ist, und den angeregten Zustand auf diese Weise stabilisieren. Daher findet eine stabilisierende Energieabsenkung des angeregten Zustandes durch die Delokalisierung der beiden Elektronen statt, was eine Umordnung der Bindungen zur Folge hat, die letztlich zur protontransferierten Form des Moleküls führt (vgl. Abb. 2.4).

Da sich die beteiligten $\pi$-Orbitale über die Gesamtheit des aromatischen Ringsystems erstrecken und die berechnete Verschiebung des Wasserstoffatoms sehr klein ist, folgerten Nagaoka und Nagashima, dass es sich bei dem übertragenen Teilchen nicht um ein Proton, sondern eher um ein Wasserstoffatom handelt, und ESIPT letztlich eher eine Deformation des aromatischen Ringgerüstes darstellt, als eine alleinige Bewegung des Wasserstoffkerns. Hieraus schlossen sie, dass die strukturelle Reorganisation in ESIPT nicht innerhalb des Wasserstoffbrücken-gebundenen Bereiches stattfindet, sondern eher andere Freiheitsgrade des Moleküls an ihr beteiligt sind. Darüberhinaus betonten sie, dass ein durch den Transfer eines Protons resultierendes Zwitterion wegen der Coulombanziehung sehr wahrscheinlich instabil wäre. Den Einfluss der Polarität des Lösungsmittels auf das Fluoreszenzspektrum führten Nagaoka und Nagashima auf die unterschiedlichen Dipolmomente des $\mathrm{S}_{1}$ und $\mathrm{S}_{1}^{\prime}$-Zustandes zurück.

Die Betrachtung der Wellenfunktionen liefert auch eine weitere Bestätigung für die Annahme, dass die Wahrscheinlichkeit für einen doppelten Wasserstoffatomtransfer bei 1.8-DHAQ sehr klein ist, da die zugehörige Wellenfunktion im angeregten Zustand zwei Knotenebenen besitzen würde, was diesen Zustand wesentlich instabiler macht als den angeregten Zustand nach einfachem Wasserstoffatomtransfer. 


\section{Kapitel 3}

\section{Experimente}

\subsection{Lasersystem}

Das Lasersystem, mit dem die Experimente durchgeführt wurden, besteht aus den folgenden fünf Komponenten (vgl. Abb. 3.1):

- Argon-Ionen cw-Pumplaser (Sabre Innova 25 von Coherent)

- modengekoppelter Titan-Saphir Laseroszillator (Mira 900 von Coherent)

- regenerativer Titan-Saphir Verstärker (RegA 9000 von Coherent)

- Gitter-Strecker/-Kompressor

Der Argon-Ionen cw-Pumplaser läuft in einem Multilinien-Betrieb im blau-grünen Wellenlängenbereich von $457 \mathrm{~nm}$ bis $515 \mathrm{~nm}$. Von der insgesamt 21 Watt GesamtPumpleistung wird mit ca. 7 Watt der Titan-Saphir Laseroszillator gepumpt und mit ca. 14 Watt der Titan-Saphir Verstärker.

Der Titan-Saphir Laseroszillator nutzt die so genannte Kerrlinsen-Modenkopplung, um ultrakurze Pulse im fs-Bereich zu erzeugen. Die Zentralwellenlänge der erzeugten Pulse ist durchstimmbar. Die im Experiment genutzten Zentralwellenlängen liegen bei $790 \mathrm{~nm}$ bis $800 \mathrm{~nm}$, die Bandbreiten betragen ca. $35 \mathrm{~nm}$, woraus Pulsbreiten von etwa 30 fs resultieren. Die Repetitionsrate beträgt $76 \mathrm{MHz}$ und die Energie pro Puls etwa 16 nJ.

Der Gitter-Strecker dient der zeitlichen Verbreiterung der Oszillatorpulse vor dem Eintritt in den Verstärker. Hierdurch wird verhindert, dass hohe Spitzenleistung zur Schädigung des Verstärkerkristalls führen.

Der Titan-Saphir Verstärker verstärkt die aus dem Gitter-Strecker kommenden Pulse, in ca. 24 Rundläufen auf etwa $4 \mu \mathrm{J}$ pro Puls. Ein akustooptischer $\mathrm{TeO}_{2}{ }^{-}$ Cavity-Dumper ermöglicht die Ein- und Auskoppelung einzelner Pulse mit einer 
Repetitionsrate von $250 \mathrm{kHz}$. Zur Steigerung der Effizienz sorgt ein akustooptischer Q-Switch dafür, dass der Laserprozess solange unterdrückt wird, bis der aus dem Oszillator kommende Puls injiziert wurde. Daneben bewirkt die Dispersion in dem $\mathrm{TeO}_{2}$-Q-Switch-Kristall während der wiederholten Umläufe eine Pulsverbreiterung bis zu 40 ps. Hierdurch wird zusätzlich verhindert, dass hohe Spitzenleistung zur Schädigung des Verstärkerkristalls führen.

Nach dem Austritt aus dem Verstärker wird die im Strecker und dem Q-Switch eingeführte Dispersion im Gitter-Kompressor wieder komprimiert. Die resultierenden Pulse haben Breiten von etwa 40 fs.

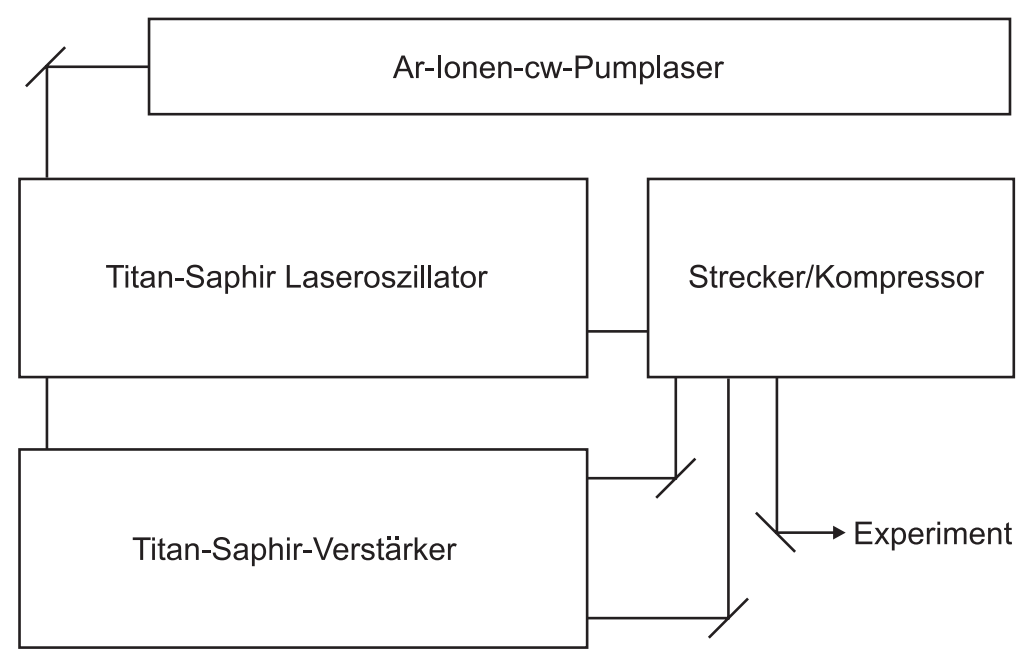

Abbildung 3.1: Das eingesetzte Lasersystem. 


\subsection{Fluoreszenz-Aufkonvertierungs-Experiment}

Im folgenden Kapitel wird zunächst kurz das Prinzip eines Fluoreszenz-Aufkonvertierungs-Experiments erklärt. Darauf folgt eine Zusammenstellung der für die Durchführung eines solchen Experiments wesentlichen Fakten aus der Theorie der Summenfrequenzerzeugung. Im Anschluss daran wird die im Rahmen dieser Arbeit aufgebaute experimentelle Anordnung zur Durchführung des Versuchs vorgestellt. In den letzten Unterkapiteln wird die Probenpräparation, insbesondere die Deuterierung des von 1.8-DHAQ, und schließlich der Ablauf der Messungen beschrieben.

\subsubsection{Prinzip}

In einem FLUC-Experiment (Fluorescence Up-Conversion Experiment) wird die in einer Probe durch einen ultraschnellen Laserpuls angeregte Fluoreszenz der Frequenz $\omega_{F}$ mit einem weiteren ultraschnellen Laserpuls, dem so genannten Gatepuls der Frequenz $\omega_{G}$, in einem nichtlinearen Kristall überlagert und so die Summenfrequenz $\omega_{S}$ erzeugt.

$$
\omega_{S}=\omega_{F}+\omega_{G}
$$

Die Intensität bei der Summenfrequenz $\omega_{S}$ wird als Funktion der Verzögerung des Gatepuls gegenüber dem Anregungspuls detektiert. Hierdurch erhält man den zeitlichen Verlauf der Fluoreszenz. Der Aufkonvertierungsprozess in dem nichtlinearen Kristall fungiert folglich als optisches Fenster bei der Beobachtung des zeitlichen Verlaufs der Fluoreszenz (vgl. Abb. 3.2).

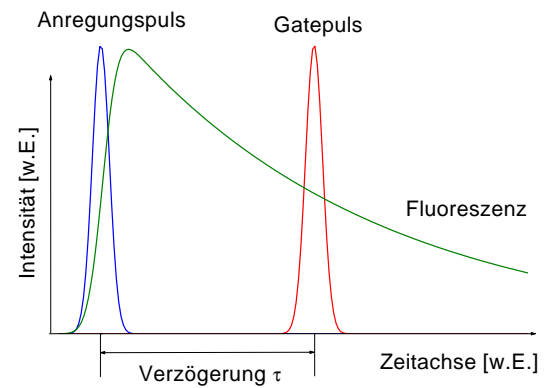

Abbildung 3.2: Prinzip eines FLUC-Experiments: Erzeugung und Aufkonvertierung der Fluoreszenz mittels Laserpulse ermöglicht eine Beobachtung der Entwicklung der Fluoreszenz auf einer durch die Pulsdauern bestimmten Zeitskala.

Aufgrund der Tatsache, dass der Mischprozess nur stattfindet, solange der Gatepuls zeitlich und räumlich mit der Fluoreszenz überlappt, kann auf diese Weise eine Zeitauflösung in der Größenordnung der Gatepulsdauer erzielt werden. Da für eine effektive Aufkonvertierung überdies Phasenanpassungsbedingungen 
erfüllt sein müssen (vgl. Kap. 3.2.2), kann abhängig von den Eigenschaften des verwendeten Kristalls auch eine moderate Frequenzselektion erreicht werden. Die Intensität des Summenfrequenzsignals $I_{S}$ zu einer bestimmten Verzögerungszeit $\tau$ ist ${ }^{1}$ proportional zur Korrelationsfunktion der Fluoreszenzintensität $I_{F}$ mit der Intensität des Gatestrahls $I_{G}[49]$.

$$
I_{S}(\tau) \propto \int_{-\infty}^{+\infty} I_{F}(t) \cdot I_{G}(t-\tau) d t
$$

\subsubsection{Theorie der Summenfrequenzerzeugung Phasenanpassung und Signalstärke}

Eine effiziente Summenfrequenzerzeugung kann nur dann stattfinden, wenn im nichtlinearen Kristall bestimmte Phasenanpassungsbedingungen von den beteiligten Strahlen erfüllt werden. Abhängig von der Frequenz des Gatepulses und dem Winkel $\theta$ zwischen den einfallenden Strahlen und der optischen Achse des Kristalls (sog. Phasenanpassungswinkel) kann nur ein bestimmter, enger Frequenzbereich des Fluoreszenzlichtes aufkonvertiert werden. Letztlich folgen die Phasenanpassungsbedingungen aus der grundlegenden Forderung nach Energieund Impulserhaltung. Mit den Frequenzen $\omega_{F}, \omega_{G}, \omega_{S}$ und den Wellenvektoren $\overrightarrow{k_{F}}$, $\overrightarrow{k_{G}}, \overrightarrow{k_{S}}$ der beteiligten Fluoreszenz(F)-, Gatepuls(G)- und Summenfrequenz(S)Photonen lauten die entsprechenden grundlegenden Gleichungen

$$
\omega_{F}+\omega_{G}=\omega_{S}
$$

und

$$
\overrightarrow{k_{F}}+\overrightarrow{k_{G}}=\overrightarrow{k_{S}}
$$

Für den im Experiment genutzten Fall kollinearer Strahlen reduziert sich Gleichung $3.4 \mathrm{zu}$

$$
\frac{n_{F}}{\lambda_{F}}+\frac{n_{G}}{\lambda_{G}}=\frac{n_{S}}{\lambda_{S}}
$$

wobei $\mathrm{n}_{F / G / S}$ den Brechungsindex für die entsprechenden Wellenlängen $\lambda_{F / G / S}$ darstellt. Die Abhängigkeit der Brechungsindizes von der Wellenlänge findet man in der Literatur in Form der Sellmeier-Gleichungen [50]. In einem uniaxialen Kristall, wie dem im Experiment genutzten BBO-Kristall, hängt der Brechungsindex überdies von der Polarisation und der Ausbreitungsrichtung des einfallenden Lichtes ab. Für Strahlen deren Polarisationsvektor senkrecht zur optischen Achse des Kristalls liegt (sog. ordentliche Strahlen=o-Strahlen), gilt unabhängig von ihrer Richtung der so genannte ordentliche Brechungsindex $n_{o}$. Für Strahlen anderer

\footnotetext{
${ }^{1}$ im Limit kleiner Konversionseffizienzen
} 
Polarisationen (sog. außerordentliche Strahlen=e-Strahlen) hängt der Brechungsindex $n_{e}(\theta)$ vom Winkel $\theta$ zwischen ihrer Ausbreitungsrichtung und der optischen Achse des Kristalls ab und berechnet sich nach [51] aus

$$
\frac{1}{n_{e}^{2}(\theta)}=\frac{\sin ^{2} \theta}{n_{e}^{2}}+\frac{\cos ^{2} \theta}{n_{o}^{2}}
$$

wobei $n_{o}$ den ordentlichen und $n_{e}$ außerordentlichen Brechungsindex für die Ausbreitung vertikal bzw. parallel zur optischen Achse bezeichnen. Für den im Experiment vorliegenden Fall der TypI-Summenfrequenzerzeugung, bei dem zwei o-Strahlen der Wellenlängen $\lambda_{F}$ und $\lambda_{G}$ zu einem e-Strahl der Wellenlängen $\lambda_{S}$ konvertieren, ergibt sich für kollineare Strahlen aus den Gleichungen 3.5 und 3.6 der Phasenanpassungswinkel $\theta$ zu

$$
\sin ^{2}(\theta)=\frac{n_{S}^{-2}(\theta)-n_{o, S}^{-2}}{n_{e, S}^{-2}-n_{o, S}^{-2}}
$$

mit

$$
n_{S}^{2}(\theta)=\lambda_{S}\left(\frac{n_{o, F}}{\lambda_{F}}+\frac{n_{o, G}}{\lambda_{G}}\right) .
$$

In Abb. 3.3 wird die Phasenanpassung noch einmal graphisch veranschaulicht.

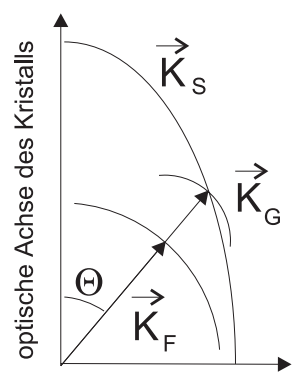

Abbildung 3.3: Phasenanpassungsgeometrie im Falle der kollinearen Typ IWechselwirkung.

Für den verwendeten BBO-Kristall liegen die Phasenanpassungswinkel zwischen $37.8^{\circ}$ für die Summenfrequenzbildung von $500 \mathrm{~nm}$ und $800 \mathrm{~nm}$ und $32.4^{\circ}$ für das Mischen von $650 \mathrm{~nm}$ und $800 \mathrm{~nm}$. Zu beachten ist beim Durchstimmen der aufkonvertierten Fluoreszenzwellenlänge durch Drehung des Kristalls, dass der letztlich benötigte Einfallswinkel $\alpha$ gemäß dem Snellius'schen Brechungsgesetz durch

$$
\alpha=\arcsin \left(n_{o, F / G} \cdot \sin (\theta-\vartheta)\right)
$$

gegeben ist, sofern der Kristall so geschnitten ist, dass bei einem senkrechten Einfall der Winkel $\vartheta$ zwischen dem Strahl und der optischen Achse liegt. 
Die größte Konversionseffizienz wird erzielt, wenn die obigen Phasenanpassungsbedingungen erfüllt werden. Die Intensität des Summenfrequenzsignals $I_{S}$ ist in diesem Fall proportional zur Intensität der Fluoreszenz $I_{F}$ und der des Gatestrahls $I_{G}$, und nimmt quadratisch mit der Dicke L des nichtlinearen Kristalls $\mathrm{zu}$ [52]. Bei Auftreten einer Abweichung $\Delta \vec{k}=\vec{k}_{S}-\vec{k}_{F}-\vec{k}_{G} \neq 0$, reduziert sich das Summenfrequenzsignal für $\mathrm{I}_{F} \ll \mathrm{I}_{G}$ gemäß

$$
I_{S} \propto I_{F} \cdot I_{G} \cdot L^{2} \cdot \frac{\sin ^{2}(\Delta k L / 2)}{(\Delta k L / 2)^{2}} .
$$

Für $\Delta k=\pi / L$ beträgt die Signalintensität folglich nur noch etwa $60 \%$ der phasenangepassten Intensität.

\section{Akzeptanzwinkel und spektrale Bandbreite}

Ein $\Delta k \neq 0$ kann prinzipiell auf zwei Arten eingeführt werden. Zum einem durch eine Abweichung von den phasenangepassten Wellenlängen, zum anderen durch eine Abweichung von dem phasenangepassten Winkel. Bei der Fokussierung des eingesammelten Fluoreszenzlichtes auf den Aufkonvertierungs-Kristall muss also beachtet werden, dass eine effiziente Summenfrequenzerzeugung nur innerhalb eines gewissen Akzeptanzwinkels stattfindet. Für den vorliegenden Fall der winkeldurchstimmbaren, kollinearen Phasenanpassung in einem uniaxialen Kristall ergibt sich der Akzeptanzwinkel $\Delta \theta_{\|}$in der Hauptebene ${ }^{2}$ des Kristalls nach [53] aus

$$
\Delta \theta_{\|}=\frac{\pi}{L}\left(\frac{\partial k_{S}}{\partial \theta}\right),
$$

wobei $\partial k_{S} / \partial \theta=\left(2 \pi / \lambda_{S}\right)(\partial n / \partial \lambda)$ und die Abhängigkeit des Brechungsindizes von $\theta$ aus Gleichung 3.8 folgt. Der Akzeptanzwinkel $\theta_{\perp}$ in der Ebene senkrecht dazu berechnet sich in der Kleinwinkelnäherung nach

$$
\Delta \theta_{\perp}=\sqrt{2 \pi / L k_{F}\left(1-k_{F} / k_{S}\right)} .
$$

Hierbei wurde der Akzeptanzwinkel als derjenige Winkel festgelegt, der eine Phasenfehlanpassung von $\Delta k=\pi / L$ verursacht, und somit die Intensität auf etwa $60 \%$ reduziert (s.o.). Zu beachten ist, dass es sich bei den angegeben Winkeln um die im Kristall auftretenden Winkel handelt. Infolge der Brechung an der Oberfläche des Kristalls vergrößern sich diese gemäß dem Snellius'schen Brechungsgesetz. In Kleinwinkelnäherung ist $\Delta \theta_{\text {extern }}=n \cdot \Delta \theta$, wenn $\mathrm{n}$ der Brechungsindex des betrachteten Strahls ist.

\footnotetext{
${ }^{2}$ Definiert als diejenige Ebene, die die optische Achse des Kristalls und den einfallenden Lichtstrahl enthält. Identisch mit der Ebene, in der der Phasenanpassungswinkel eingestellt wird.
} 
Untersucht man analog dazu, ab welchen Frequenzabweichungen das Summenfrequenzsignal auf 40\% seiner Maximalintensität abweicht, erhält man die spektrale Bandbreite $\delta \omega$ gemäß [53]

$$
\Delta \omega=\pi\left(2 L\left(\left.\frac{\partial k_{S}}{\partial \omega}\right|_{\omega_{S}}-\left.\frac{\partial k_{F}}{\partial \omega}\right|_{\omega_{F}}\right)\right)^{-1}
$$

wobei $\partial k / \partial \omega=(n-\lambda(\partial n / \partial \lambda)) / c$ und die Abhängigkeit der Brechungsindizes von $\lambda$ in der Literatur in der Form so genannter Sellmeier-Gleichungen zu finden ist.

\section{Zeitauflösung}

Da eine Summenfrequenzerzeugung nur stattfindet, solange der Gatepuls zeitlich mit der Fluoreszenz überlappt, entspräche die Zeitauflösung eines FluoreszenzUpconversion-Experiments im Idealfall der Pulsdauer $\Delta \tau_{G}$ des Gatepulses. Diese wird jedoch schon durch die einfache Tatsache verschlechtert, dass die Fluoreszenz nicht durch einen $\delta$-förmigen Laserpuls ausgelöst wird, sondern der Anregungspuls bereits eine endliche Dauer $\Delta \tau_{A}$ besitzt, die Fluoreszenz selbst also schon zeitlich 'verschmiert' ist (vgl. Abb. 3.4 und 3.5).

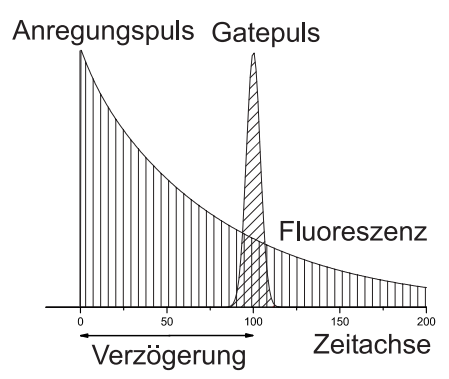

Abbildung 3.4: Fluoreszenzverlauf bei Anregung mittels $\delta$-Puls.

Sind die Gatepulse sehr kurz, also im Frequenzraum relativ breitbandig, kann es zu einer weiteren Verschlechterung der Zeitauflösung kommen, sofern die Frequenzbandbreite der Summenfrequenzerzeugung in dem verwendeten Kristall kleiner ist als die Frequenzbandbreite des Gatepulses. In diesem Fall erfolgt die Aufkonvertierung mit einem Gatepuls effektiv geringerer Bandbreite und somit nach dem Fourierlimit von längerer Dauer. Zu einer größtenteils vermeidbaren Verringerung der Zeitaufösung kommt es auch dann, wenn die zur Pulsverbreiterung führende Gruppengeschwindigkeitsdispersion (GVD), die der Anregungspuls in der Probenlösung und der Gatepuls im Aufkonvertierungs-Kristall erfährt, nicht durch eine optimal justierte Prismenkombination vorkompensiert wurden. Allerdings kann auf diese Weise nur die GVD 1.Ordnung kompensiert werden, höhere 


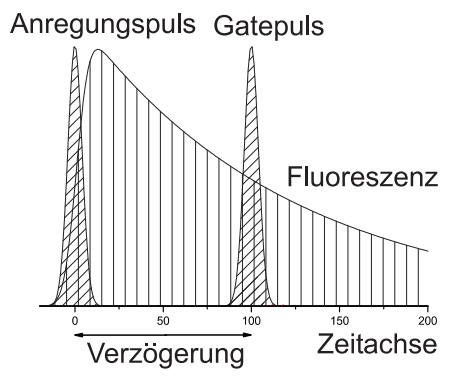

Abbildung 3.5: Fluoreszenzverlauf bei Anregung mit einem Laserpuls endlicher Breite.

Ordnungen sind nicht korrigierbar. Die Dispersion die die Fluoreszenz in Probenlösung, Zellenfenster, Filter und Kristall erfährt, spielt für die Zeitauflösung des hier durchgeführten Experiments keine Rolle, da nur ein sehr kleiner Frequenzbereich von $\pm 6 \mathrm{~nm}$ (Monochromator-Auflösung $\approx \pm 2 \mathrm{~nm}$ ) betrachtet wird. Einen ganz entscheidenden Einfluss auf die erreichbare Zeitaufösung hat jedoch die Größe des Unterschieds zwischen den Gruppengeschwindigkeiten des Gatepulses und der Fluoreszenz. Indem die beiden Strahlen aneinander vorbeilaufen, überstreicht und konvertiert der Gatepuls einen größeren zeitlichen Dynamikbereich der Fluoreszenzkurve als der Gatepulsdauer entspricht (vgl. Abb. 3.6) Die hieraus resultierende Verschlechterung der Zeitauflösung berechnet sich nach [51]

$$
\Delta t=L\left(\frac{1}{v_{G}}-\frac{1}{v_{F}}\right)
$$

wobei

$$
v_{G / F}=\left|\left(\frac{\partial \vec{k}_{G / F}}{\partial \omega}\right)\right|^{-1}=c\left(n_{G / F}-\left.\lambda_{G / F}\left(\frac{\partial n_{G / F}}{\partial \lambda}\right)\right|_{\lambda_{G / F}}\right)^{-1} .
$$

Gleichung 3.14 zeigt, dass bei 'normaler' Dispersion die Zeitauflösung mit zunehmender Fluoreszenzwellenlänge zunimmt (bei konstant gehaltener Gatewel-

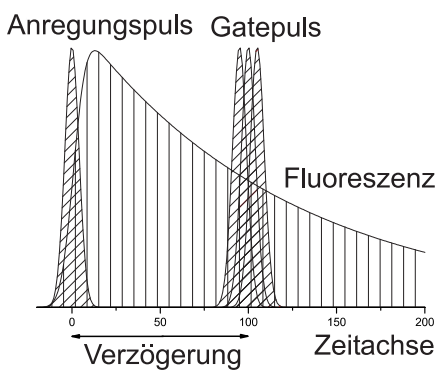

Abbildung 3.6: Verschlechterung der Zeitauflösung aufgrund unterschiedlicher Gruppengeschwindigkeiten des Gatepulses und der Fluoreszenz. 
lenlänge $\lambda_{G}>\lambda_{F}$ ). Damit wird verständlich, weshalb die Bestimmung der Kreuzkorrelation zwischen dem gestreuten Anregungslicht und dem Gatepuls nur eine Abschätzung der untersten Grenze der Zeitauflösung liefern kann.

\subsubsection{Experimenteller Aufbau}

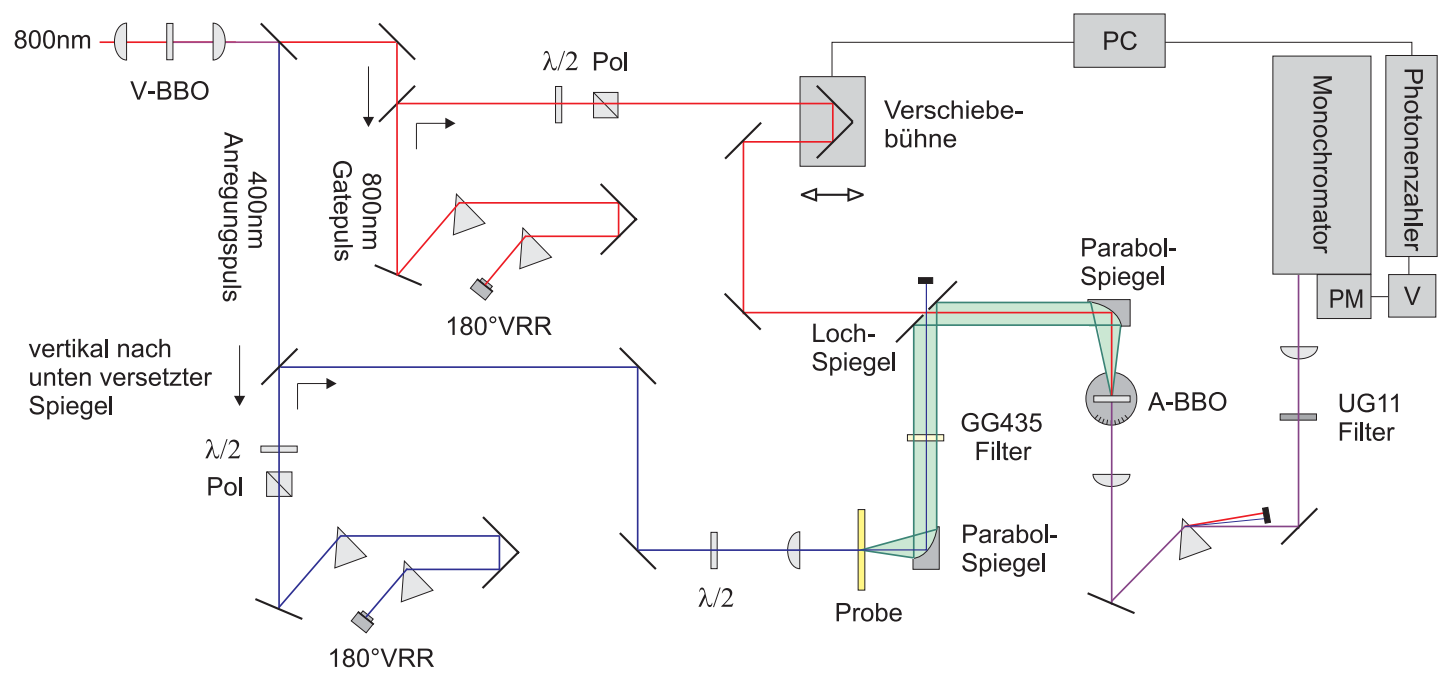

Abbildung 3.7: Schematischer Aufbau des FLUC-Experiments. $V$-BBO = Verdoppler-BBO, A-BBO = Aufkonvertierungs-BBO, $P M=$ Photomultiplier, $V$ $=$ Verstärker, $180^{\circ}$ VRR $=180^{\circ}$ Rückreflektor mit vertikalem Strahlversatz

Der schematische Versuchsaufbau für das im Rahmen dieser Arbeit aufgebaute FLUC-Experiment ist in Abbildung $3.7 \mathrm{zu}$ sehen. Als Lichtquelle dienen die bei einer Zentralwellenlänge von $800 \mathrm{~nm}$ in einem Ti-Saphir-System erzeugten Pulse mit einer Bandbreite von etwa $38 \mathrm{~nm}$, die einer Pulsdauer von ungefähr $\simeq 40 \mathrm{fs}$ entspricht. Die Repetitionsrate beträgt $250 \mathrm{kHz}$ und die mittlere Leistung liegt bei etwa $1300 \mathrm{~mW}$.

Die aus dem Lasersystem kommenden Lichtpulse werden mittels eines BBOKristalls (Typ I, $\mathrm{d}=0.5 \mathrm{~mm}, \theta=32^{\circ}$ ) frequenzverdoppelt. Ein nachfolgender dielektrischer Spiegel (390 nm HR) trennt die transmittierte Fundamentale bei $800 \mathrm{~nm}$, die als Gatepuls fungiert, von der reflektierten Verdoppelten bei $400 \mathrm{~nm}$, die als Anregungspuls genutzt werden wird. In beiden nachfolgenden Strahlengängen befinden sich Doppelpass-Prismenkombinationen (Suprasil) zur Vorkompensation der Gruppengeschwindigkeitsdispersion 2.Ordnung, die die beiden Strahlen beim Durchlaufen der experimentellen Anordnung erfahren. Zur Reduktion der Reflexionsverluste erfolgt der Eintritt in die Prismen unter dem Brewsterwinkel der jeweiligen Wellenlänge. Ebenfalls in beiden Strahlengängen befindet sich eine 
Kombination aus $\lambda / 2-$ Plättchen und Polarisator, mittels der die Polarisation der Strahlen geeignet gedreht und ihre Pulsenergie bei Bedarf abgeschwächt werden kann. Hierzu muss, da der aus dem Lasersystem kommende Gatepuls p-polarisiert ist, und somit der durch Verdopplung aus ihm hervorgegangene Anregungspuls s-polarisiert, die Polarisation des letzteren vor dem Durchlaufen der Prismenkombinationen um $90^{\circ}$ gedreht werden.

Mittels eines weiteren $\lambda / 2-$ Plättchen wird die Polarisation des Anregungspulses noch ein weiteres Mal gedreht, um eine Anregung unter dem so genannten 'magischen Winkel' von 54.8 relativ zur Polarisation des Gatepulses zu ermöglichen. Unter diesen Bedingungen ist das Abklingverhalten des Signals unabhängig von einer Emissionsanisotropie der untersuchten Probe, verursacht durch die Reorientierungsbewegungen der Probenmoleküle in Lösung [54].

Mittels einer Quarzlinse ( $\mathrm{f}=50 \mathrm{~mm}$ ) wird der Anregungspuls schließlich auf die Probe (freier Strahl der Lösung bzw. Rotationszelle, vgl. Kap. 3.4.4) fokussiert. Die Fluoreszenz angeregten Probenmoleküle wird mit einem aluminiumbeschichteten Parabolspiegel ( $90^{\circ}$ off-axis, $\mathrm{f}=50.8 \mathrm{~nm}$, Janos) eingefangen und parallelisiert. Ein nachfolgender Frequenzfilters (Schott GG435, d=1 mm) unterdrückt das Streulicht des Anregungsstrahls. Der dann folgende Spiegel im Fluoreszenzstrahlengang besitzt ein Loch in seinem Zentrum. Zum einen entweicht das Restlicht des nun nicht mehr benötigte Anregungspuls hierdurch, und zum anderen wird durch dieses der Gatepuls parallel zum Fluoreszenzlicht in den Strahlengang eingekoppelt. Anschließend werden dann beide Strahlen durch einen weiteren Parabolspiegel (90 off-axis, $\mathrm{f}=101.6 \mathrm{~mm}$, Janos) auf den BBO-Kristall (Typ I, $\mathrm{d}=1 \mathrm{~mm}, \theta=31^{\circ}$ ) fokussiert. In diesem kommt es zur Summenfrequenzerzeugung zwischen Gatepuls- und Fluoreszenzphotonen.

Im Experiment kommen von der Kollimation der Fluoreszenz bis zur Aufkonvertierung keine Linsen sondern Reflektionsoptiken zum Einsatz, da diese keine chromatischen Aberrationen erzeugen. Dies ist wichtig, um einerseits den gesamten $\mathrm{zu}$ untersuchenden Frequenzbereich der Fluoreszenz zwischen $470 \mathrm{~nm}$ und $670 \mathrm{~nm}$ auf einen Fokus abbilden zu können, und andererseits, um mit Hilfe des zweiten Parabolspiegels den um 800 nm zentrierten Gatepuls und die Fluoreszenz gleichzeitig auf denselben Punkt im BBO-Kristall fokussieren zu können.

Der BBO-Kristall befindet sich auf einem Rotationstisch mit einer Winkelskala, der ein Verstellen des Kristallwinkels zur Änderung der phasenangepassten Fluoreszenzwellenlänge ermöglicht (vgl. Kap. 3.2.2).

Damit die Verzögerung des Gatepuls in Bezug auf den Anregungspuls verändert werden kann, befindet sich im Strahlengang des Gatepulses eine computergesteuerte Translationsbühne (Melles Griot Nanomotion II, max.Translation $25 \mathrm{~mm}$ ). 
Das Summenfrequenzsignal wird durch eine Quarzlinse kollimiert und beim Durchlaufen eines Quarzprismas und eines Frequenzfilters (Schott UG11, d=2 mm) vom Gatepuls, dessen nicht-phasenangepasster zweiter und dritter Harmonischen, und der Restfluoreszenz selbst getrennt. Schließlich wird es mittels eines GitterMonochromators (0.5 m Ebert Scanning Spectrometer Model 82-000) spektral zerlegt $(\Delta \lambda= \pm 2 \mathrm{~nm}$ ) und auf einen Photomultiplier (Hamamatsu R928 bei den Messungen am Jet bzw. 1P28 bei den Messungen in der Zelle) abgebildet. Das hier erzeugte elektrische Signal wird 40 -fach verstärkt und anschließend in einen Photonenzähler (SR-400 Stanford Research Systems SI) geleitet. Zur Unterdrückung des Untergrundrauschens wird die Höhe der Signalschwelle des Photonenzählers optimiert, und darüberhinaus sein Betriebsmodus so gewählt, dass nur Photonen innerhalb eines begrenzten Zeitfensters nach dem Triggern durch den Gatepuls registriert werden. Photonenzähler und Verschiebebühne werden über eine GPIBSchnittstelle vom Computer durch ein hierfür geschriebenes HPVEE-Programm angesteuert.

\subsubsection{Parameter des Messvorgangs}

Im Experiment wurde jeweils bei fester Wellenlänge das aufkonvertierte Fluoreszenzsignal in Abhängigkeit von der zeitlichen Verzögerung des Gatepuls gegenüber dem Anregungspuls gemessen. Die Selektion der Wellenlänge erfolgte durch ein Optimieren der Winkel des BBO-Kristalls und des Prismas im Detektionsstrahlengang auf ein maximales Signal der am Monochromator eingestellten Wellenlänge. Alle FLUC-Messungen wurden bei Raumtemperatur durchgeführt.

Der Ablauf der FLUC-Messungen am Probenlösungsstrahl verlief wie folgt: Es wurden pro Wellenlänge jeweils 3 Einzelkurven aufgenommen. Die Schrittweite der Verschiebebühne war $20 \mathrm{fs}$ im Bereich von -1 ps bis 10 ps und $150 \mathrm{fs}$ im Bereich von 10 ps bis 100 ps. Die Messdauer pro Punkt betrug je nach Wellenlänge $0.5 \mathrm{~s}$ bis $1 \mathrm{~s}$, was bei einer Laserrepetitionsrate von $250 \mathrm{kHz}$ einer Aufsummation der durch 125000-250000 Anregungsprozesse erzeugten Photonen entspricht. Die durchschnittliche Anregungsleistung lag bei $100 \mathrm{~mW}$ und die Gatepulsleistung bei $108 \mathrm{~mW}$. Die Schichtdicke des Probenlösungsstrahls war etwa $0.1 \mathrm{~mm}$.

Der Ablauf der FLUC-Messungen an der Zelle verlief wie folgt: Es wurden pro Wellenlänge jeweils 6 bis 12 Einzelkurven aufgenommen. Die Schrittweite der Verschiebebühne war $25 \mathrm{fs}$ im Bereich von -1 ps bis 3 ps, $200 \mathrm{fs}$ im Bereich von 3 ps bis 10 ps und 1 ps im Bereich von 10 ps bis 100 ps. Die Messdauer pro Punkt betrug $1 \mathrm{~s}$. Die durchschnittliche Anregungsleistung lag bei $0.95 \mathrm{~mW}$ und die Gatepulsleistung bei $15 \mathrm{~mW}$. Die Schichtdicke des Lösungsmittels in der Zelle war etwa $0.4 \mathrm{~mm}$. 


\subsection{Pump-Probe-Experiment}

Im folgenden Kapitel wird kurz das Prinzip eines klassischen Pump-Probe-Experiments erläutert. Anschließend wird die im Rahmen dieser Arbeit aufgebaute experimentelle Anordnung zur Durchführung des Versuchs vorgestellt und der Verlauf der Messungen beschrieben.

\subsubsection{Prinzip}

Für ein klassisches Pump-Probe-Experiment werden mindestens zwei ultrakurze Lichtpulse benötigt. Mit dem so genannten Pumppuls wird die Substanz angeregt. Mit dem zweiten als Probepuls bezeichneten Lichtpuls, der gegenüber dem Pumppuls zeitlich um t verzögert wird, wird die Änderung in der betrachteten Substanz zum Zeitpunkt t nach der Anregung untersucht. Hierzu wird beispielsweise die Intensität des Probepulses nach Durchqueren der Substanz mit und ohne Anregung gemessen $\left(\mathrm{I}\left(\omega_{\text {Probe }}, \mathrm{t}\right)\right.$ und $\left.\mathrm{I}_{0}\left(\omega_{\text {Probe }}\right)\right)$ und miteinander verglichen. Je nach der Frequenz des Probepulses wird seine Intensität in einem Fall beim Durchgang durch die angeregte Substanz infolge transienter Absorption aus dem angeregten Zustand in höher liegende Zustände geschwächt, und im anderen Fall infolge eines Ausbleichen ${ }^{3}$ des Grundzustands des Systems oder durch stimulierte Emission verstärkt. Nach dem Lambert-Beerschen-Gesetz gilt

$$
I\left(\omega_{\text {Probe }}, t\right)=I_{0}\left(\omega_{\text {Probe }}\right) \cdot 10^{-\epsilon_{\omega} N(t) L},
$$

wobei $\epsilon_{\omega}$ der Absorptionskoeffizient der untersuchten Substanz bei der Probenpulsfrequenz $\omega$ ist, $\mathrm{N}(\mathrm{t})$ die Population der Ausgangsniveaus darstellt und L die Weglänge, die der Probepuls in der Substanz zurücklegt. Die physikalische Größe von Interesse ist jedoch die optischen Dichte (vgl. [55]). Sie ist definiert als

$$
O D\left(\omega_{\text {Probe }}, t\right)=-\log \frac{I\left(\omega_{\text {Probe }}\right)}{I_{0}\left(\omega_{\text {Probe }}\right)}=\epsilon_{\omega} N(t) L .
$$

Betrachtet man also bei einer festen Beobachtungsfrequenz $\omega$ die Änderung der optischen Dichte mit der Verzögerungszeit t gewinnt man einen direkten Einblick in die Populationsdynamik der untersuchten Substanz nach der Anregung.

\subsubsection{Experimenteller Aufbau}

Als Lichtquelle dient das bereits vorgestellte Ti-Saphir-Lasersystem, das Pulse bei einer Zentralwellenlänge von $800 \mathrm{~nm}$ mit einer Bandbreite von etwa $38 \mathrm{~nm}$

\footnotetext{
${ }^{3}$ Von einem Ausbleichen des Grundzustands spricht man, wenn infolge der Anregung die Population im Grundzustand verringert ist und daher Wellenlängen die im Bereich des Absorptionsspektrums liegen weniger stark absorbiert werden, als es ohne vorherige Anregung der Fall wäre.
} 
und einer Pulsdauer von $\simeq 40 \mathrm{fs}$ erzeugt. Die Repetitionsrate beträgt $250 \mathrm{kHz}$, und die mittlere Leistung etwa $1300 \mathrm{~mW}$.

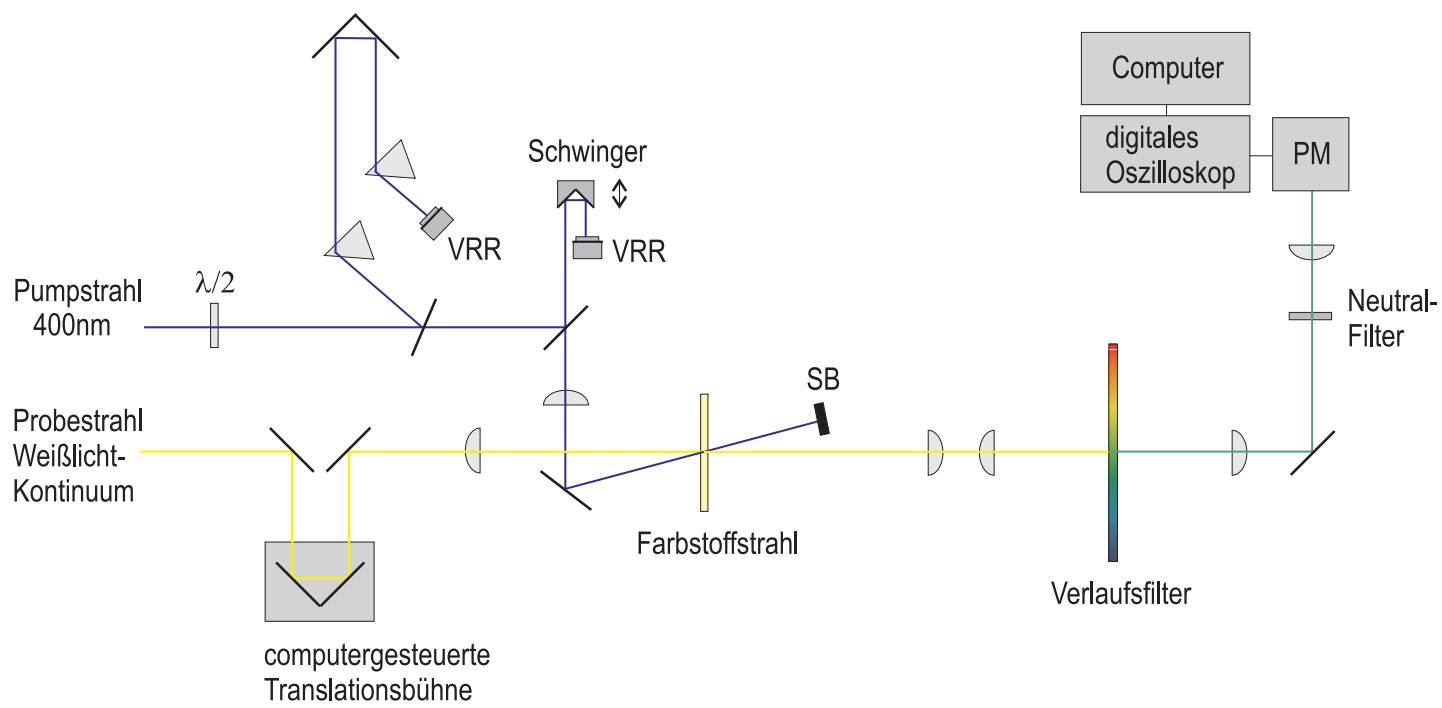

Abbildung 3.8: Schematischer Aufbau des Pump-Probe-Experiments. PM =Photomultiplier, $S B=$ Strahlblocker, Schwinger $=$ vor und zurück schwingender $180^{\circ}$ Rückreflektor mit horizontalem Strahlversatz. VRR $=180^{\circ}$ Rückreflektor mit vertikalem Strahlversatz

Ein Teil der aus dem Lasersystem kommenden Pulse wird mittels eines BBOKristalls (Typ I, $\mathrm{d}=0.5 \mathrm{~mm}, \theta=32^{\circ}$ ) frequenzverdoppelt. Diese um $400 \mathrm{~nm}$ herum zentrierten Pulse dienen der Anregung der Probenlösung und werden im folgenden als Pumppulse bezeichnet. Mit dem anderen Teil der Laserpulse wird in einem Saphirkristall Weißlicht erzeugt, das als Probepuls dient. Ein schematischer Versuchsaufbau für das Pump-Probe-Experiment ist in Abbildung $3.8 \mathrm{zu}$ sehen. Im Strahlengang des Pumpstrahls befindet sich zunächst eine DoppelpassPrismenkombination aus Suprasil zur Vorkompensation der Gruppengeschwindigkeitsdispersion 2.Ordnung, die der Strahl beim Durchlaufen der experimentellen Anordnung erfährt. Zur Reduktion der Streulichtverluste erfolgt der Eintritt in die Prismen unter dem Brewsterwinkel. Hierzu muss die Polarisation des durch Frequenzverdopplung erzeugten, s-polarisierten Pumppulses vor dem Durchlaufen der Prismenkombinationen mit Hilfe eines $\lambda / 2$-Plättchens um $90^{\circ}$ gedreht werden.

Damit die Verzögerung des Probepuls in Bezug auf den Pumppuls verändert werden kann, befindet sich im Strahlengang des Pumppulses ein mit einer Frequenz von $10 \mathrm{~Hz}$ horizontal vor und zurück schwingender $180^{\circ}$-Rückreflektor. Der schwingende Rückreflektor bringt den Vorteil einer schnellen Datenaufnahme mit 
$\operatorname{sich}^{4}$. Er hat jedoch in der verwendeten Konstruktion ${ }^{5}$ den Nachteil, dass für die Rekonstruktion der realen Zeitskala vor und nach der eigentlichen Messung eine Eichkurve aufgenommen werden muss, da der schwingende Rückreflektor zwischen den Umkehrpunkten in seiner Bewegung beschleunigt wird. Zwischen der Messung und der Aufnahme der Eichkurve darf keine Änderung am experimentellen Aufbau stattfinden. Eine weitere Einschränkung ist, dass mit der verwendeten Schwinger-Konstruktion nur ein Zeitbereich von etwa $22 \mathrm{ps}$ betrachtet werden kann. Um eine Eichung der Zeitskala durchführen zu können, befindet sich im Strahlengang des Probestrahls eine computergesteuerte Translationsbühne ${ }^{6}$.

Mittels zweier Quarzlinsen $(\mathrm{f}=150 \mathrm{~mm}$ ) werden Pumppuls und Probepuls unter einem Winkel von etwa $30^{\circ}$ schließlich auf einen frei strömenden Strahl der Probenlösung von etwa $300 \mu \mathrm{m}$ Dicke fokussiert. Der Winkel zwischen den beiden Strahlen ermöglicht es, den Pumpstrahl aus dem Detektionsstrahlengang zu entfernen. Aus dem Probepuls wird nach dem Durchqueren des Jets mit Hilfe eines Verlauffilters ein Frequenzband einer Breite von etwa $10 \mathrm{~nm}^{7}$ selektiert und nach Abschwächung durch Neutralgraufilter auf einen Photomultiplier (Hamamatsu R928) geschickt, dessen Signal zur Messdatenerfassung zu einem digitalen Oszilloskop geleitet wird. Das digitale Oszilloskop wird durch den schwingenden $180^{\circ}$-Rückreflektor getriggert.

\subsubsection{Parameter des Messvorgangs}

Gemessen wurde das Pump-Probe-Signal jeweils bei fester Proben-Wellenlänge in Abhängigkeit von der Verzögerung des Probepulses gegenüber dem Pumppuls. Beobachtet wurde bei Verzögerungszeiten von -1 ps bis 22 ps in einer Schrittweite von 4-10 fs. Diese Schrittweite war nicht äquidistant, da der vor und zurück schwingende $180^{\circ}$-Rückreflektor eine beschleunigte Bewegung ausführt, und daher die Anzahl der Messpunkte im Bereich der Umkehrpunkte größer ist als in den übrigen Bereichen. Die aufgenommenen Kurven mussten daher erst über eine Eichkurve in die Realzeit übertragen werden. Die gemessenen Signale entsprechen dem Mittel über 10000 Perioden des vor und zurück schwingenden $180^{\circ}$-Rückreflektors.

Die durchschnittliche Pumpleistung am Ort des Jets lag bei $70 \mathrm{~mW}$ und die Probeleistung bei $17 \mathrm{~mW}$.

\footnotetext{
${ }^{4}$ Die "Rapid-Scan"-Technik wurde bereits in verschiedenen anderen Experimenten eingesetzt $[56,57]$

${ }^{5}$ Schwinger $=180^{\circ}$-Rückreflektorspiegel, der über Federn durch ein exzentrisches Rad bewegt wird.

${ }^{6} 25 \mathrm{~mm}$ Translationsbühne: Melles Griot Nanomotion II

${ }^{7}$ Die Selektion der Wellenlänge sowie das Abschätzen der vom Strahldurchmesser auf dem Filter abhängigen Bandbreite erfolgte mit Hilfe eines REES-Spektrometers.
} 


\subsection{Probenpräparation und Probenhalterung}

\subsubsection{Probenlösungen}

In allen Messungen wurde 1.8-DHAQ (Aldrich) nach Reinigung mittels SilicaGel-Spaltenchromatographie verwendet. Als Lösungsmittel dienten Benzylalkohol, Propylencarbonat und Tetrachlorkohlenstoff. Die Konstitutionsformeln der eingesetzten Lösungsmittel sind in Abbildung 3.9 skizziert. Die Konzentration der Probenlösung für den Jet wurde so gewählt, dass die optische Dichte der Probe bei $400 \mathrm{~nm}$ und einer Jetschichtdicke von etwa $100 \mu \mathrm{m}$ bei $\mathrm{OD}=0.3 \mathrm{lag}$. Die benötigte Lösung hierfür war etwa $5 \cdot 10^{-3}$-molar. Die Konzentration der Lösung für die Rotationszelle wurde jeweils so gewählt, dass die optische Dichte (vgl. Gl. 3.17) der Probe für $400 \mathrm{~nm}$ bei $\mathrm{OD}=1$ lag. Die hierfür benötigten Lösungen waren $10^{-3}$ bis $10^{-2}$-molar. Eine Dimerisierung kann ausgeschlossen werden, da die stationären Absorptions- und Emissionsspektren bei diesen Konzentrationen identisch waren mit denen von $10^{-4}$-molaren Lösungen.

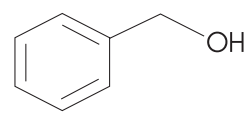

Benzylalkohol $\mathrm{C}_{7} \mathrm{H}_{8} \mathrm{O}$

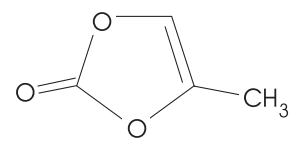

Propylencarbonat $\mathrm{C}_{4} \mathrm{H}_{6} \mathrm{O}_{3}$

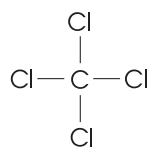

Tetrachlorkohlenstoff $\mathrm{CCl}_{4}$

Abbildung 3.9: Konstitutionsformeln der verwendeten Lösungsmittel.

\subsubsection{Deuterierung}

Die Substitution der Hydroxylgruppen-Protonen von 1.8-DHAQ durch Deuterium gelang durch wiederholten Austausch der gesättigten Probenlösung in $\mathrm{CCl}_{4}$ mit $\mathrm{D}_{2} \mathrm{O}$ und jeweils etwa einstündigem Durchmischen der Emulsion. Der Deuterierungsgrad von 87-93\% ergab sich sowohl durch Analyse von IR-Spektren des eingedampften und gepressten deuterierten Derivats von 1.8-DHAQ $(\mathrm{OH}-$ /OD-Peakintensitätsvergleich) als auch durch Auswertung von NMR-Spektren in Lösung (OH-/H-Peakintensitätsvergleich). Zur Vermeidung einer Entdeuterierung erfolgt die Abfüllung der Lösung in eine geschlossene Rotationsmesszelle unter wasserfreier Stickstoffatmospäre. Vor und nach jeder Messung wurde durch Aufnahme eines Absorptionsspektrum der Probenlösung in der verwendeten Zelle der qualitative Bestand der Deuterierung kontrolliert. 


\subsubsection{Frei fließender Probenlösungsstrahl}

Die Messungen in Benzylalkohol und Propylencarbonat wurden an einem frei fließenden Strahl der Probenlösung durchgeführt. Der Vorteil des freien Strahls besteht zum einen in der hohen erreichbaren Durchflussgeschwindigkeit, die eine Anregung von 1.8-DHAQ mit größerer Intensität erlaubt, ohne dass dieses hierdurch zerstört würde. Dies hat wiederum zur Folge, dass das Signal-zu-RauschVerhältnis der am freien Probenlösungsstrahl gemessenen Kurven sehr viel besser ist, als bei Kurven, die im gleichen Zeitraum an einer Probenzelle aufgenommen wurden. Zum anderen erfährt die im FLUC-Experiment erzeugte Fluoreszenz aufgrund der fehlenden Fenster keine stärkere Dispersion bis zu ihrer Detektion, was eine bessere Zeitauflösung im Vergleich zu Messungen an Probenzellen zur Folge hat. Für das Pump-Probe-Experiment kommt als weiterer positiver Aspekt des freien Probenlösungsstrahls hinzu, dass, da keine dispersiven Fenster wie bei Probenzellen vorhanden sind, etwaige durch Kreuzphasenmodulation verursachte Artefakte nur noch vom Lösungsmittel verursacht werden können.

\subsubsection{Geschlossene Rotationszelle}

Auch eine Rotationszelle besitzt ihre unbestreitbaren Vorzüge. Zum einen wird aufgrund des geringen Zellenvolumens sehr viel weniger Probenlösung als für einen frei fließenden Strahl benötigt. Zum anderen ermöglicht die geschlossene Zelle die Verwendung leicht flüchtiger Lösungsmittel, wie z.B. Tetrachlorkohlenstoff. Die Möglichkeit eine Rotationszelle hermetisch zu verschießen ist auch eine entscheidende Voraussetzung für die Verwendung deuterierter Derivate von 1.8DHAQ, die durch den Kontakt mit Feuchtigkeit der Umgebungsluft entdeuteriert würden.

\subsection{Messung stationärer Spektren}

Die für eine spektrale Normierung der Fluoreszenzintensitäten benötigten stationären Emissionsspektren (vgl. Kap. 4.1.3) wurden an einem Fluoreszenz-Spektrometer von Jobin-Yvon (Fluorolog-3) in $10^{-4}$-molaren Lösungen bei Raumtemperatur aufgenommen.

Die zur qualitativen Kontrolle des Deuterierungsgrades aufgenommenen stationären Absorptionsspektren wurden mit einem UV/VIS-Spektrometer von Shimadzu (UV-160) direkt in der Probenlösung der Rotationszelle bei Raumtemperatur aufgenommen. Die übrigen stationären Absorptionsspektren wurden in $10^{-4}$-molaren Lösungen durchgeführt. 


\section{Kapitel 4}

\section{Ergebnisse und Datenanalyse}

In diesem Kapitel werden die experimentell ermittelten Ergebnisse vorgestellt und die zur Analyse der Daten verwendeten Methoden besprochen. Der Übersichtlichkeit halber erfolgt eine Unterteilung entsprechend den verwendeten Lösungsmitteln Benzylalkohol, Propylencarbonat und Tetrachlorkohlenstoff. Es wird jeweils ein kurzer Überblick über die stationären Spektren von 1.8-DHAQ in diesen Lösungsmitteln gegeben und anschließend die aus dem FLUC-Experiment ermittelte Fluoreszenzdynamik präsentiert. Pump-Probe-Transienten wurden nur in Benzylalkohol gemessen. In Tetrachlorkohlenstoff wurde darüberhinaus noch der Einfluss der Deuterierung von 1.8-DHAQ auf die stationären Spektren und die Fluoreszenzdynamik untersucht.

\subsection{8-DHAQ in Benzylalkohol}

\subsubsection{Stationäre Spektren in Benzylalkohol}

Abbildung 4.1 zeigt das bei Raumtemperatur aufgenommene stationäre Absorptions- und Emissionsspektrum von 1.8-DHAQ in Benzylalkohol. Das Absorptionsspektrum zeigt kaum Struktur und besitzt lediglich zwei schwach ausgeprägte Schultern zu beiden Seiten des Maximums bei etwa $436 \mathrm{~nm}$. Diese werden wahrscheinlich durch Anregung hochfrequenter Schwingungsmoden verursacht $[35,46]$. Das Emissionsspektrum wurde nach Anregung bei $400 \mathrm{~nm}$ aufgenommen. Seine Form weicht merklich von einem einfachen Spiegelbild des Absorptionsspektrums ab, obgleich sich auch hier nicht viel Struktur im Spektrum zeigt. Neben dem Maximum bei etwa $596 \mathrm{~nm}$ ist nur eine schwache Schulter bei etwa $520 \mathrm{~nm} \mathrm{zu}$ beobachten. Der Stokesshift zwischen dem Absorptions- und Emissionsmaximum ist mit $\simeq 6160 \mathrm{~cm}^{-1}$ deutlich ausgeprägt. 


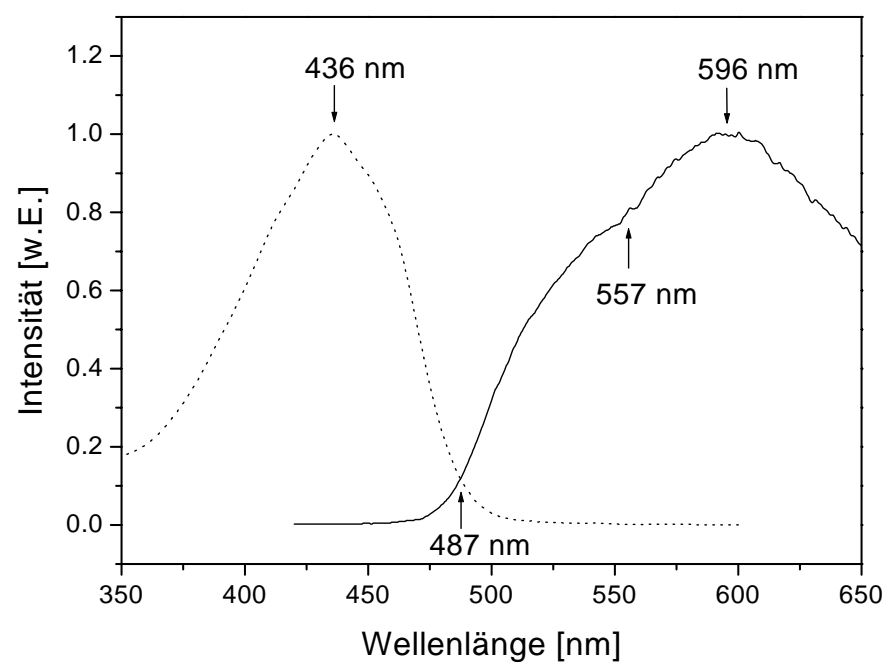

Abbildung 4.1: Stationäres Absorptionsspektrum (gestrichelte Kurve) und Emissionsspektrum (durchgezogene Kurve) nach Anregung bei $400 \mathrm{~nm}$ von 1.8-DHAQ in Benzylalkohol.

\subsubsection{Pump-Probe-Transienten in Benzylalkohol}

In Abbildung 4.2 sind experimentell ermittelte Pump-Probe-Signalkurven für verschiedene Probenwellenlängen abgebildet. Aufgetragen ist die durch den Pumppuls ausgelöste Änderung des Transmissionsverhaltens von 1.8-DHAQ in Benzylalkohol in Abhängigkeit von der Verzögerung des Probepulses gegenüber dem Pumppuls. Die Wellenlänge des Pumppulses lag bei $400 \mathrm{~nm}$. Als Probepuls diente das Licht eines Weißlichtkontinuums. Die geprobten Wellenlängen lagen im Bereich von $500 \mathrm{~nm}$ bis $680 \mathrm{~nm}$. Alle Pump-Probe-Messungen wurden bei Raumtemperatur durchgeführt.

Für Probenwellenlängen unterhalb von etwa $570 \mathrm{~nm}$ ist eine transiente Absorption zu beobachten, oberhalb davon findet man stimulierte Emission. Ein Ausbleichen des Grundzustandes kann als Ursache für eine verringerte optische Dichte ausgeschlossen werden, da die gewählten Probenwellenlängen außerhalb des stationären Absorptionsspektrums liegen. Die Intensität der transienten Absorption und auch der stimulierten Emission steigt innerhalb der ersten $300 \mathrm{fs}$ bis $400 \mathrm{fs}$ an und fällt danach monoton ab. In beiden Wellenlängenbereichen zeigt sich eine periodische Modulation der Signalintensität. Im Bereich des zeitlichen Ursprungs, in dem Pump- und Probepuls einander überlappen, wird das Signal stark von einem Kreuzphasenmodulations-Artefakt beeinflusst.

Ursache für das Kreuzphasenmodulations-Artefakt ist die Intensitätsabhängig- 


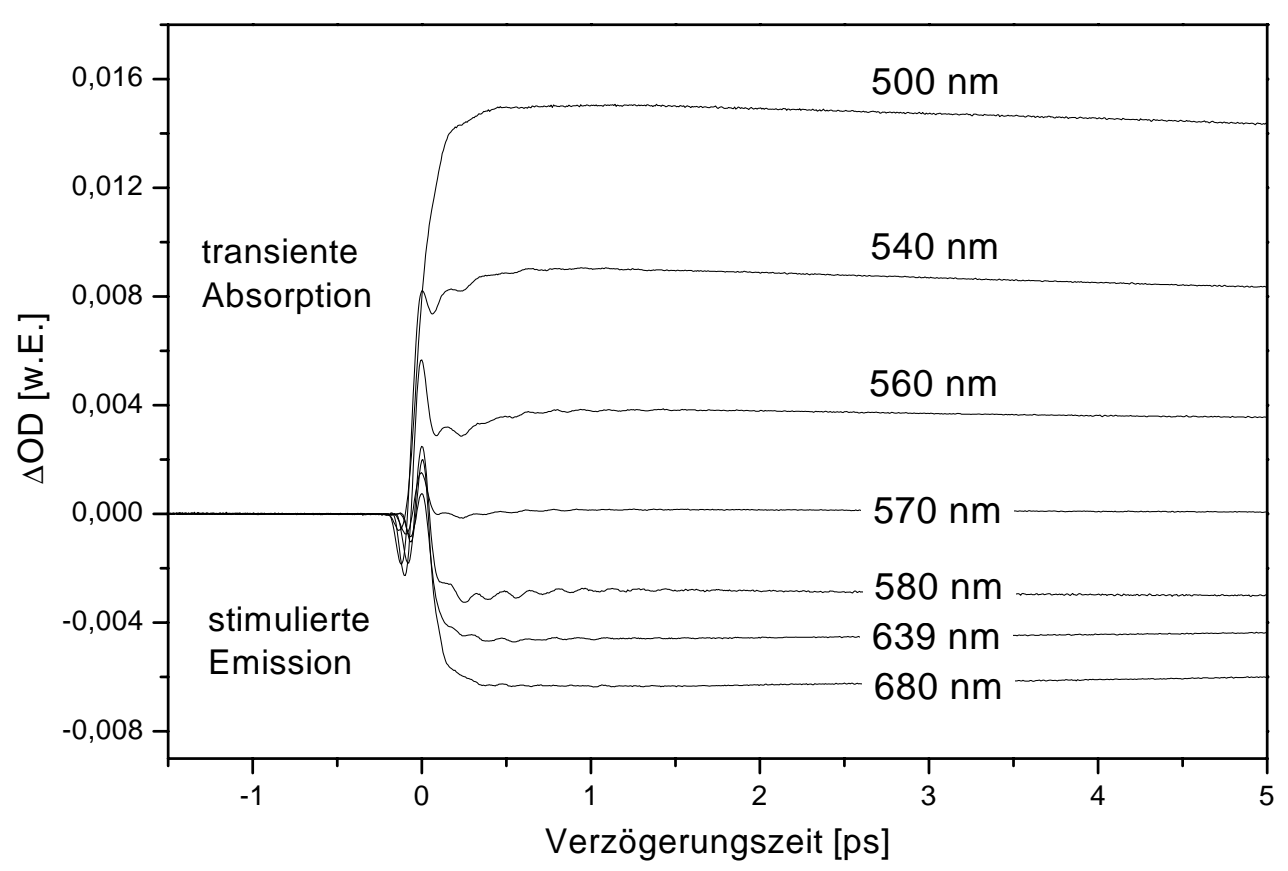

Abbildung 4.2: Pump-Probe-Signale von 1.8-DHAQ in Benzylalkohol.

keit des Brechungsindex bei hohen Intensitäten $\mathrm{I}(\mathrm{t})$. Der starke Pumppuls bewirkt daher während seiner Dauer eine zeitliche Änderung des Brechungsindex des Mediums gemäß $\mathrm{n}(\mathrm{t})=\mathrm{n}_{0}-\mathrm{n}_{2} \mathrm{I}(\mathrm{t})$, wobei $\mathrm{n}_{0}$ und $\mathrm{n}_{2}$ den linearen bzw. nichtlinearen Brechungsindex des Mediums darstellen. Diese Änderung des Brechungsindex führt bei dem schwächeren Probepuls zu einer zeitlichen Änderung seiner Phase gemäß $\phi(\mathrm{t}) \propto \mathrm{n}(\mathrm{t}) \omega / \mathrm{c}$, was wiederum nach $\Delta \omega=-(\partial \phi / \partial t)$ eine Änderung seiner instantanen Frequenz zur Folge hat. In Kombination mit dem Gruppengeschwindigkeitsunterschied zwischen dem Probepuls und den verschiedenen spektralen Komponenten des gechirpten Weißlicht-Probepulses führt dies zu einer zeitlichen Modulation der detektierten Transmission des Probepulses, da sich im Detektionsstrahlengang ein Frequenz-Filter befindet [58].

Das reine Kreuzphasenmodulations-Artefakt zwischen dem Pumppuls und dem gechirpten Weißlicht-Probepuls, ohne den Einfluss von 1.8-DHAQ in einem nichtlinearen Kristall ${ }^{1}$ aufgenommen, zeigt für alle hier gemessenen Probenwellenlängen eine nahezu spiegelsymmetrische W-Form (vgl. Abb. 4.3). Dieses entspricht auch bereits bekannten experimentellen Beobachtungen und theoretischen Vor-

\footnotetext{
${ }^{1} 0.5 \mathrm{~mm}$ dicker BBO bei nicht-phasenangepasstem Winkel für die Summenfrequenzerzeugung.
} 


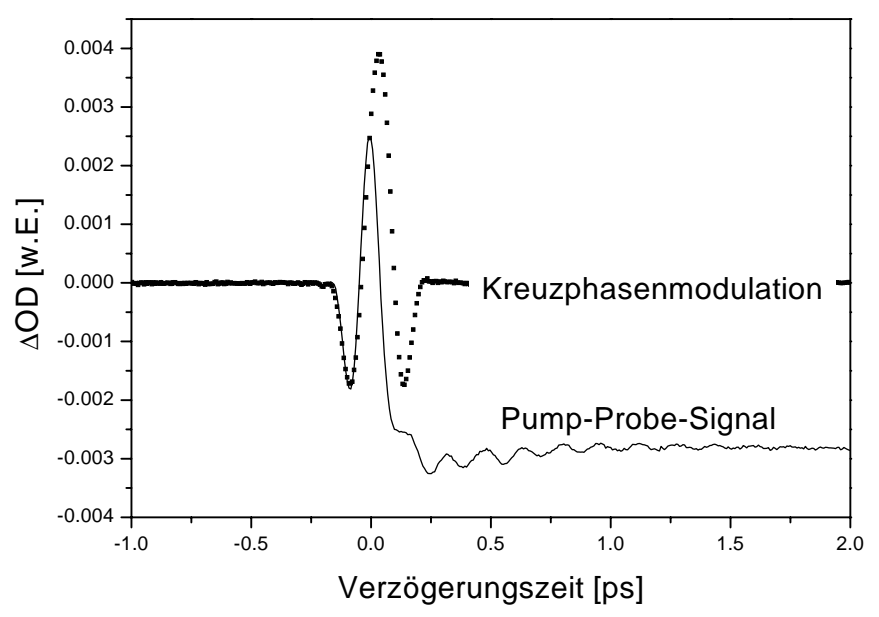

Abbildung 4.3: Zeitlicher Verlauf der Kreuzphasenmodulation gemessen in einem BBO-Kristall und des Pump-Probe-Signals von 1.8-DHAQ in Benzylalkohol. Die Wellenlänge des Pumppulses lag bei $400 \mathrm{~nm}$, der Weißlicht-Probepuls wurde mit einem Monochromator bei $580 \mathrm{~nm}$ detektiert.

hersagen [58]. In Benzylalkohol sollte sich die gleiche Form ergeben, allerdings mit veränderter Amplitude, da der nichtlineare Brechungsindex für Benzylalkohol ein anderer ist. Auch die Breite des Artefaktes kann im Experiment variieren, sofern sich die Breite des Pumppulses oder der Chirp des Probepulses bei den Messungen verändert. Hinzu kommt, dass bei Anwesenheit von 1.8-DHAQ das resultierende Signal nicht einer einfachen Überlagerung der optischen Antwort von 1.8-DHAQ und des Kreuzphasenmodulations-Artefaktes entsprechen kann, denn die Änderung der instantanen Frequenz des Probepulses müsste auch eine veränderte instantane optische Antwort von 1.8-DHAQ zur Folge haben. Über den Signalverlauf für eine feste Probenwellenlänge im Bereich des zeitlichen Pulsüberlapps kann daher keine eindeutige Aussage gemacht werden. Aus diesem Grund wird dieser Zeitbereich bei der Analyse der Daten nicht weiter berücksichtigt. Leider kann aus demselben Grund auch der Nullpunkt nicht genauer als $\pm 20 \mathrm{fs}$ festgelegt werden, was zur Folge hat, dass die absolute Phase der beobachteten Oszillationen nicht genau bestimmt werden kann. Dies ist bedauerlich, da die Phase einen Hinweis auf den Ursprung der Oszillation geben könnte. Letztlich kann eine verlässliche Aussage nur über die Frequenzen und die Abklingzeiten der beobachteten Oszillationen gemacht werden.

Die oszillatorischen Beiträge im Pump-Probe-Signal wurden nach Abzug des nichtoszillierenden Signalbeitrags mit Hilfe einer LPSVD-Anpassung ("Linear prediction singular value decomposition") analysiert [59,60]. Als Anpassungsfunktion diente 


$$
S(t)=\sum_{i} A_{i} \cos \left(\omega_{i} t+\phi_{i}\right) \exp \left(-\frac{t}{\tau_{i}}\right)
$$

wobei $A_{i}$ die Amplitude, $\omega_{i}$ die Frequenz, $\phi_{i}$ die Phase und $\tau_{i}$ die Abklingzeit der beobachteten Oszillation ist. Zur Überprüfung der daraus erhaltenen Ergebnisse wurde zusätzlich eine Fouriertransformation der oszillatorischen Signalbeiträge durchgeführt. Aus dem Beobachtungszeitraum von etwa 22 ps lässt sich die kleinste beobachtbare sog. Nyquistfrequenz auf etwa $2 \mathrm{~cm}^{-1}$ abschätzen. Nach dem Shannonschen Abtasttheorem ist mit einer Abtastperiode von $\Delta \mathrm{t}$ die höchste erfassbare Frequenz im Messsignal 1/2 $\Delta$ t. Im Falle des Pump-Probe-Experiments sollten daher bei einer Pulsdauer von etwa $40 \mathrm{fs}$ Frequenzen bis $417 \mathrm{~cm}^{-1}$ aufzulösen sein.

\begin{tabular}{|c||c|c|c|c|}
\hline$\lambda_{\text {Probe }}$ & $\begin{array}{c}\tau \text { bei } \\
{[\mathrm{nm}]}\end{array}$ & $\begin{array}{c}\tau \text { bei } \\
{[\mathrm{fs}]}\end{array}$ & $\begin{array}{c}\tau \text { bei } \\
(330-350) \mathrm{cm}^{-1} \\
{[\mathrm{fs}]}\end{array}$ & $\begin{array}{c}\begin{array}{c}\tau \text { bei } \\
(70) \mathrm{cm}^{-1} \\
{[\mathrm{fs}]}\end{array} \\
(30-60) \mathrm{cm}^{-1} \\
{[\mathrm{fs}]}\end{array}$ \\
\hline \hline 540 & $730-740$ & - & $240-250$ & - \\
\hline 560 & $740-800$ & 200 & $120-130$ & $350-540$ \\
\hline 570 & $720-790$ & - & - & - \\
\hline 580 & $830-980$ & - & - & $280-370$ \\
\hline 605 & $640-680$ & $610-850$ & $230-310$ & $240-270$ \\
\hline 621 & $630-700$ & $500-870$ & $180-580$ & $250-290$ \\
\hline 639 & $630-750$ & $640-840$ & - & $150-200$ \\
\hline
\end{tabular}

Tabelle 4.1: Abklingzeiten $\tau$ der beobachteten Oszillation im Pump-Probe-Signal von 1.8-DHAQ in Benzylalkohol bei verschiedenen Probewellenlängen $\lambda_{\text {Probe }}$.

Zu beobachten sind die Oszillationen bei Probewellenlängen zwischen $520 \mathrm{~nm}$ und $660 \mathrm{~nm}$, wobei ihre Amplitude in den Randbereichen schwächer wird. In Tabelle 4.1 sind die Ergebnisse der LPSVD-Anpassung zusammengestellt. Die Frequenzen in den Oszillationen lassen sich in vier Bereiche zusammenfassen. Die Unsicherheit der gefundenen Frequenzen und Abklingzeiten rührt daher, dass verschiedene Anpassungsfunktion die Daten innerhalb des Rauschens der Signale gleich gut beschreiben. Die in allen beobachteten Oszillationen dominierende Frequenz liegt bei (207-215) $\mathrm{cm}^{-1}$ und klingt nach der LPSVD-Analyse mit einer Zeitkonstante zwischen $630 \mathrm{fs}$ und $850 \mathrm{fs}$ ab. Abhängig von der Probewellenlänge findet man in den Signalmodulationen auch Frequenzen bei $(30-60) \mathrm{cm}^{-1},(90-170) \mathrm{cm}^{-1}$ und (330-350) $\mathrm{cm}^{-1}$. Die Intensität dieser Beiträge ist jedoch um den Faktor 0.3 bis 0.1 schwächer als bei $(207-215) \mathrm{cm}^{-1}$. Darüberhinaus ist die Existenz der niedrigfrequenten Beiträge zu den Oszillationen bei Frequenzen von $30-60 \mathrm{~cm}^{-1}$ und 90-170 $\mathrm{cm}^{-1}$, deren Schwingungsdauern im Bereich ihrer Dämpfung und darüber 

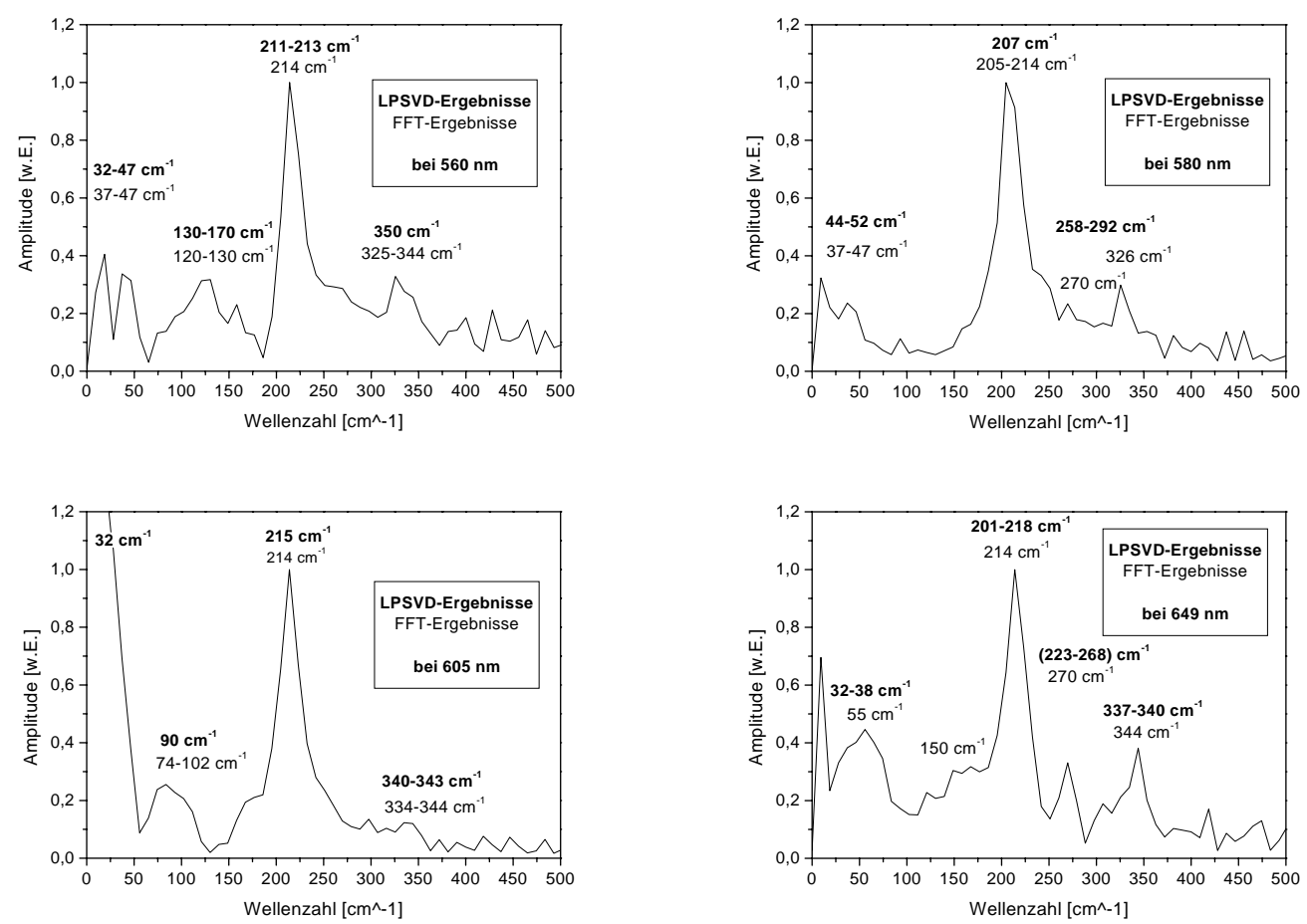

Abbildung 4.4: Vergleich der Ergebnisse der LPSVD- und FFT-Analyse der beobachteten Oszillation im Pump-Probe-Signal von 1.8-DHAQ in Benzylalkohol bei verschiedenen Probewellenlängen.

liegen, nicht signifikant, da ihre Bestimmung kritisch von der Wahl des nichtoszillierenden Signalbeitrags und der Bestimmung des Nullpunkts abhängt.

Abbildung 4.4 veranschaulicht wie die in der LPSVD-Anpassung gefundenen Frequenzen auch in den Ergebnissen der FFT-Analyse zu finden sind. Die maximale Frequenzaufösung in der FFT-Analyse beträgt $9 \mathrm{~cm}^{-1}$. Wegen eines Signal-zuRausch-Verhältnisses von nur etwa 10 ist Bestimmung der Frequenz der Maxima der bis zu $50 \mathrm{~cm}^{-1}$ breiten Linien im FFT-Spektrum jedoch nur mit einer sehr viel geringeren Genauigkeit möglich. Für das Maximum bei $214 \mathrm{~cm}^{-1} \mathrm{im}$ FFTSpektrum wurde die Lebensdauer mittels der Heisenberg'schen Unschärferelation $\Delta \mathrm{E} \Delta \mathrm{t} \geq \hbar$ über die spektrale Breite des Peaks auf (300-440) fs abgeschätzt. Sie ist wesentlich kleiner als die in der LPSVD-Anpassung erhaltenen Abklingzeiten. In einigen FFT-Spektren treten Peaks bei $0 \mathrm{~cm}^{-1}$ auf. Diese werden durch einen restlichen Offset im angepassten oszillatorischen Anteil des Signals verursacht. 


\subsubsection{Fluoreszenzdynamik in Benzylalkohol}

Mit Hilfe des FLUC-Experiments wurde die zeitaufgelöste Fluoreszenz von 1.8DHAQ in Benzylalkohol für verschiedene Wellenlängen zwischen $483 \mathrm{~nm}$ und $658 \mathrm{~nm}$ gemessen. Die Anregung erfolgte bei $400 \mathrm{~nm}$. In Abbildung 4.5 sind repräsentativ fünf der experimentell ermittelten FLUC-Signalkurven zusammen mit den jeweiligen Anpassungskurven abgebildet. Die Zunahme der absoluten Signalstärke mit ansteigender Wellenlänge erklärt sich aus einer kombinierten Betrachtung der stationären Emissionswahrscheinlichkeiten von 1.8-DHAQ für verschiedenen Wellenlängen und der Transmission der im Experiment verwendeten Filter, sowie der spektralen Empfindlichkeit des eingesetzten Photomultipliers.

Entsprechend der zeitlichen Entwicklung der Fluoreszenzintensität lässt sich das Spektrum in zwei Bereiche, unterhalb und oberhalb von etwa $560 \mathrm{~nm}$ einteilen.

Als Anpassungskurve für das gemessene zeitabhängige Signal S(t) diente bei den Signalen bis $544 \mathrm{~nm}$ eine triexponentiell abfallende Fluoreszenzintensität $\mathrm{F}\left(\mathrm{t}^{\prime}\right)$ multipliziert mit einer Heavyside-Funktion $\Theta\left(t^{\prime}\right)$, die mit einer Gaußfunktion als Antwortfunktion $\mathrm{G}\left(\mathrm{t}^{\prime}\right)$ des Systems gefaltet wurde.

$$
\begin{aligned}
S(t) & =\int_{t^{\prime}=-\infty}^{-\infty} \Theta\left(t^{\prime}\right) \cdot F\left(t-t^{\prime}\right) \cdot G\left(t^{\prime}\right) d t^{\prime} \\
& =\sum_{i=1}^{3} A_{\downarrow i} \int_{t^{\prime}=-\infty}^{-\infty} \Theta\left(t^{\prime}\right) \cdot \exp \left(\frac{t-t^{\prime}}{\tau_{i}}\right) \cdot \exp \left(\frac{-t^{\prime 2}}{2 \sigma^{2}}\right) d t^{\prime}
\end{aligned}
$$

Das Integral lässt sich analytisch lösen und man erhält

$$
S(t)=\sum_{i=1}^{3} A_{\downarrow} \cdot \sigma \cdot \exp \left[\left(\frac{\sigma}{2 \tau_{i}}\right)^{2}-\frac{t}{\tau_{i}}\right]\left(1-\operatorname{erf}\left(\frac{\sigma}{2 \tau_{i}}-\frac{t}{\sigma}\right)\right) .
$$

wobei $\mathrm{A}_{\downarrow i}$ die Amplitude der Komponente mit der Abklingzeit $\tau_{\downarrow i}$ ist und $\sigma$ die volle Halbwertsbreite der Antwortfunktion. Zur Anpassung der Signale bei Fluoreszenzwellenlängen oberhalb von $560 \mathrm{~nm}$ wurde eine mit der Antwortfunktion gefaltete Kombination aus ansteigenden (negative Amplituden $\mathrm{A}_{\uparrow j}$, Anstiegzeiten $\tau_{\uparrow j}$ ) und abklingenden Exponentialfunktionen (positive Amplituden $\mathrm{A}_{\downarrow i}$ ) benötigt. Die Anpassung der experimentellen Kurven erfolgte unter Zuhilfenahme eines iterativen "Nonlinear-Least-Squares-Fit"-Algorithmus.

In Tabelle 4.2 sind die auf diese Weise bestimmten Zeitkonstanten zusammengestellt. Im Anhang A ist eine vollständige Liste der ermittelten Anpassungsparameter zu finden. Im Bereich der kurzwelligen Fluoreszenz ist ein schnelles 


\begin{tabular}{|c||c|c|c|c|c|}
\hline $\begin{array}{c}\lambda_{\text {Fluoreszenz }} \\
{[\mathrm{nm}]}\end{array}$ & $\begin{array}{c}\tau_{\downarrow \text { lang }} \\
{[\mathrm{ps}]}\end{array}$ & $\begin{array}{c}\tau_{\downarrow \text { mittel }} \\
{[\mathrm{ps}]}\end{array}$ & $\begin{array}{c}\tau_{\downarrow \text { kurz }} \\
{[\mathrm{ps}]}\end{array}$ & $\begin{array}{c}\tau_{\uparrow \text { mittel }} \\
{[\mathrm{ps}]}\end{array}$ & $\begin{array}{c}\tau_{\uparrow \text { kurz }} \\
{[\mathrm{ps}]}\end{array}$ \\
\hline \hline 483 & 157 & 6.0 & 0.325 & - & - \\
\hline 489 & 227 & 6.9 & 0.312 & - & - \\
\hline 500 & 219 & 11.4 & 0.382 & - & - \\
\hline 509 & 330 & 16.8 & 0.509 & - & - \\
\hline 518 & 259 & 24.2 & 0.473 & - & - \\
\hline \hline 544 & 309 & - & 0.268 & - & - \\
\hline 570 & 279 & 15.4 & - & 15.4 & 5.6 \\
\hline \hline 580 & 362 & 20.6 & - & 5.7 & 0.102 \\
\hline 593 & 247 & 24.6 & - & 3.9 & 0.250 \\
\hline 622 & 193 & 35.0 & - & 1.3 & 0.144 \\
\hline 644 & 201 & 9.7 & - & 1.9 & 0.158 \\
\hline 658 & 179 & 5.0 & - & 0.85 & 0.159 \\
\hline
\end{tabular}

Tabelle 4.2: Abklingzeiten ( $\left.\tau_{\downarrow l a n g}, \tau_{\downarrow \text { mittel }}, \tau_{\downarrow k u r z}\right)$ und Anstiegszeiten ( $\tau_{\uparrow m i t t e l}$, $\left.\tau_{\uparrow k u r z}\right)$ der Fluoreszenzkurven von 1.8-DHAQ in Benzylalkohol, gemessen bei der Fluoreszenzwellenlänge $\lambda_{\text {Fluoreszenz }}$.

Abklingen der Intensität innerhalb der ersten (300-500) fs $\left(\tau_{\downarrow k u r z}\right)$ zu beobachten, gefolgt von einem langsameren Abklingen mit $\tau_{\downarrow \text { mittel }}$ auf der ps-Zeitskala. Demgegenüber ist im Bereich der langwelligen Fluoreszenz ein schnelles Ansteigen der Intensität innerhalb der ersten (100-200) fs $\left(\tau_{\uparrow k u r z}\right)$ zu beobachten, gefolgt von einem langsameren Ansteigen mit $\tau_{\uparrow m i t t e l}$ und nachfolgendem Abklingen mit $\tau_{\downarrow \text { mittel }}$ auf der ps-Zeitskala. Die Fluoreszenz bei Wellenlängen im Übergangsbereich von etwa $560 \mathrm{~nm}$ zeigt ein intermediäres Verhalten.

Die Zeitkonstanten $\tau_{\uparrow k u r z}$ im Bereich des fs-Anstiegs, sind mit einer größeren Unsicherheit behaftet als die Zeitkonstanten $\tau_{\downarrow k u r z}$, da die Anstiegszeiten der Fluoreszenzintensität in der gleichen Größenordnung wie der durch die endliche Breite der Antwortfunktion bedingte Signalanstieg liegen. Die Breite der Antwortfunktion des Systems ist von der betrachteten Fluoreszenzwellenlänge abhängig, und steigt mit abnehmender Wellenlänge an (vgl. Kap. 3.2.2). Die Bestimmung der Breite der Kreuzkorrelation zwischen Anregungspuls und Gatepuls kann daher nur eine Abschätzung der maximalen Breite der Antwortfunktion liefern. Bei der Anpassung der Daten an eine gefaltete multiexponentielle Anpassungskurve mit Hilfe eines iterativen "Nonlinear-Least-Squares-Fit"-Algorithmus wurde daher auch die Variation der Breite der Antwortfunktion zugelassen. Daher ist die erhaltene Anstiegskonstante $\tau_{\uparrow k u r z}$ nur auf etwa \pm 100 fs genau. 

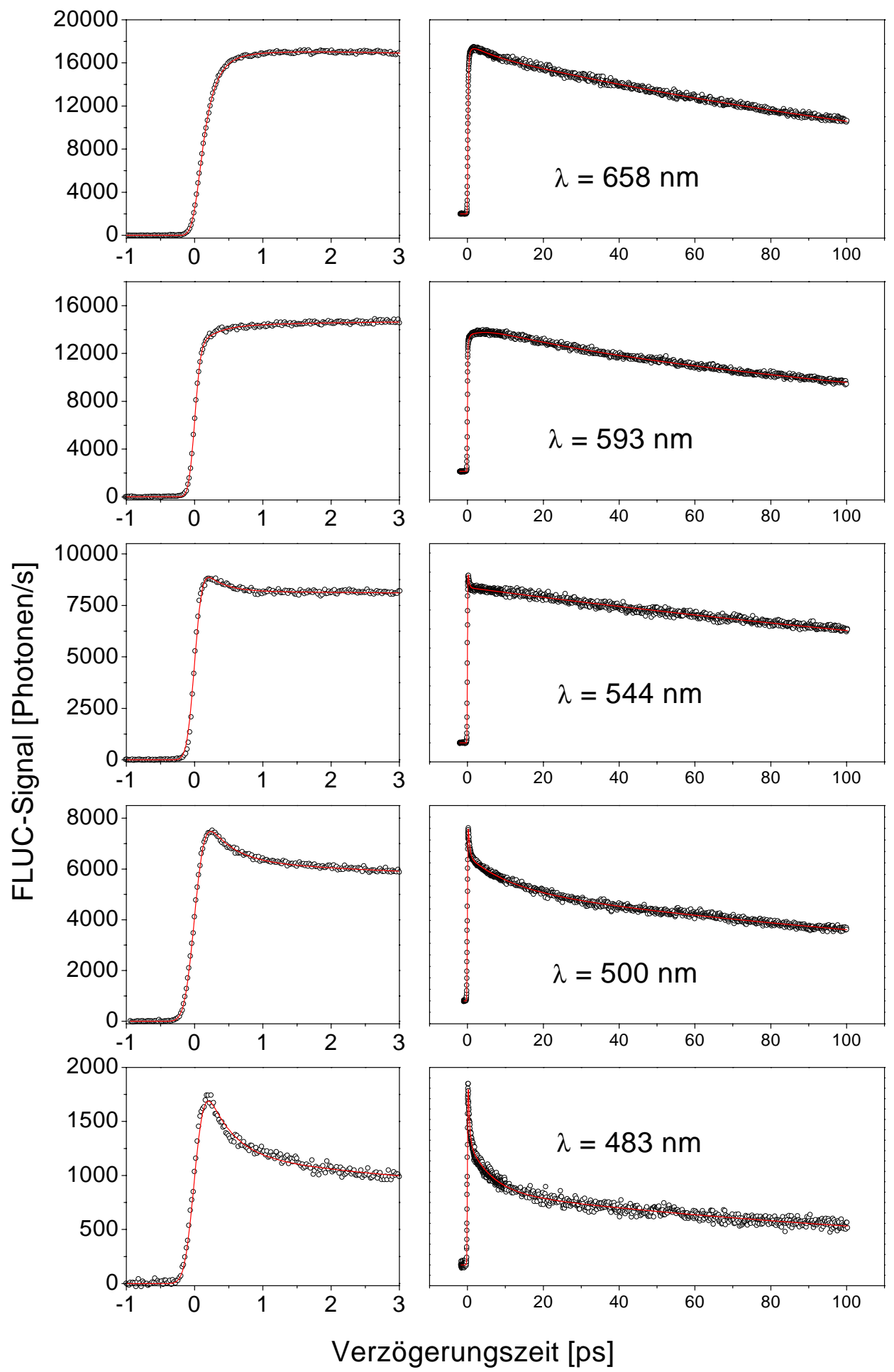

Abbildung 4.5: Zeitliche Entwicklung der Fluoreszenzintensität von 1.8-DHAQ in Benzylalkohol nach Anregung bei $400 \mathrm{~nm}$, gemessen für verschiedene Fluoreszenzwellenlängen. Experimentelle Daten sind mit offenen Kreisen, Anpassungskurven mit durchgezogenen Linie gekennzeichnet. 
Die Zeitkonstanten $\tau_{\downarrow k u r z}$ und $\tau_{\uparrow k u r z}$ zeigen im Rahmen der Genauigkeit des Experiments und der Anpassung innerhalb des langwelligen Bereichs (etwa $\pm 60 \mathrm{fs}$ ) und des kurzwelligen Bereichs (etwa $\pm 100 \mathrm{fs}$ ) keine große Abhängigkeit von der Fluoreszenzwellenlänge. Die Zeitkonstanten $\tau_{\downarrow \text { mittel }}$ und $\tau_{\uparrow \text { mittel }}$ weisen demgegenüber eine deutliche Entwicklung mit der Wellenlänge auf. $\tau_{\downarrow \text { mittel }}$ steigt im Bereich der kürzeren Fluoreszenzwellenlängen mit zunehmender Wellenlänge bis zu etwa $518 \mathrm{~nm}$ an und besitzt im Bereich der längeren Fluoreszenzwellenlängen ein Maximum zwischen $593 \mathrm{~nm}$ und $622 \mathrm{~nm}$. $\tau_{\uparrow m i t t e l}$ zeigt eine Abnahme mit zunehmender Fluoreszenzwellenlänge.

Das langsame exponentielle Abklingen der Fluoreszenz auf der Zeitskala von $\tau_{\downarrow l a n g}=(160-360)$ ps kann im Rahmen der Genauigkeit des Experiments, da die Fluoreszenz nur bis zu einem Zeitpunkt von 100 ps nach der Anregung gemessen wurde, als konstant angesehen werden, und entspricht der Lebensdauer des angeregten Zustandes von 1.8-DHAQ.

Zur Rekonstruktion der zeitabhängigen Spektren wurden die Intensitäten der mit den Anpassungsparametern aus der entsprechenden Tabelle in Anhang A gebildeten Fluoreszenzkurven auf die relative Intensität der jeweiligen Wellenlänge im stationären Spektrum normiert. Das Fluoreszenzspektrum zu einem bestimmten Zeitpunkt wurde dann durch Vergleich der Intensitäten bei verschiedenen Wellenlängen gewonnen. Die Rekonstruktion der zeitlichen Entwicklung des Fluoreszenzspektrums ist in Abbildung 4.6 abgebildet.

Bei allen beobachteten Wellenlängen setzt eine Fluoreszenzemission innerhalb der zeitlichen Auflösung des FLUC-Experiments ${ }^{2}$ von etwa (100-160) fs quasi instantan mit der Anregung von 1.8-DHAQ ein. Qualitativ ist die Form des zeitabhängigen Spektrums der des stationären Spektrums sehr ähnlich. Man beobachtet also schon 150 fs nach der Anregung eine ausschließlich stark Stokes-verschobene Emission und die Intensität im Bereich der langwelligen Fluoreszenz ist bereits stärker als die Intensität im Bereich der kurzwelligen Fluoreszenz. Allerdings ist kurz nach der Anregung der Unterschied zwischen beiden Bereichen deutlich geringer als im stationären Fall. Gut erkennbar in Abbildung 4.6 ist das Abklingen der Fluoreszenz im Bereich der kürzeren Fluoreszenzwellenlängen und das Ansteigen im Bereich der längeren Fluoreszenzwellenlängen. Der Bereich der langwelligen Fluoreszenz zeigt eine etwas größere Änderung als der Bereich der kurzwelligen Fluoreszenz.

\footnotetext{
${ }^{2}$ Abgeschätzt aus der vollen Halbwertsbreite der angepassten Antwortfunktion.
} 


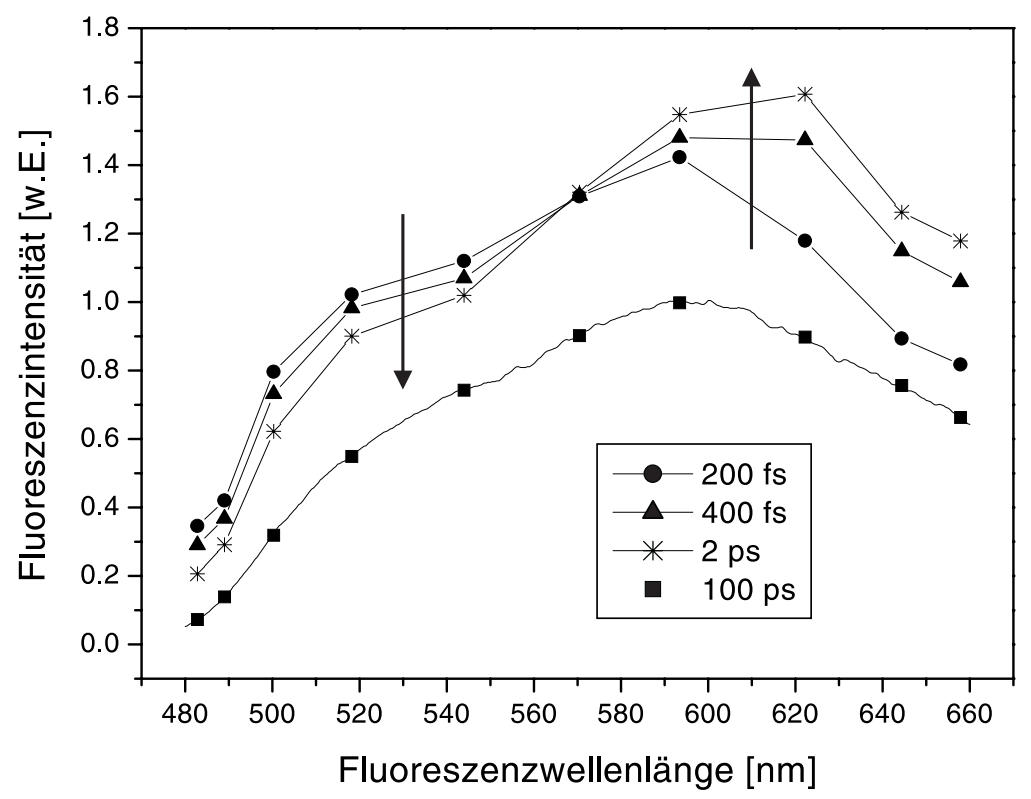

Abbildung 4.6: Zeitliche Entwicklung des Fluoreszenzspektrums von 1.8-DHAQ in Benzylalkohol. Die unterste Kurve kennzeichnet den Verlauf des stationären Spektrums.

\section{$4.2 \quad$ 1.8-DHAQ in Propylencarbonat}

\subsubsection{Stationäre Spektren in Propylencarbonat}

Abbildung 4.7 zeigt das bei Raumtemperatur aufgenommene stationäre Absorptions- und Emissionsspektrum von 1.8-DHAQ in Propylencarbonat. Die Anregung erfolgte bei $400 \mathrm{~nm}$. Das Absorptionsspektrum in Propylencarbonat ist nahezu strukturlos. Es ist lediglich eine sehr schwach ausgeprägte Schulter auf der langwelligen Seite des Maximums bei etwa $427 \mathrm{~nm}$ zu sehen.

Das wesentlich breitere Emissionsspektrum, dessen Form stark von einem einfachen Spiegelbild des Absorptionsspektrums abweicht, weist demgegenüber eine deutliche duale ${ }^{3}$ Struktur auf. Neben dem globalen Maximum bei etwa $582 \mathrm{~nm}$ ist im Bereich der kürzeren Emissionswellenlängen bei etwa $526 \mathrm{~nm}$ ein ausgeprägtes Nebenmaximum zu beobachten, sowie eine leichte Schulter bei etwa $640 \mathrm{~nm}$. Der Stokesshift zwischen dem Absorptions- und Emissionsmaximum ist mit ungefähr $6240 \mathrm{~cm}^{-1}$ deutlich ausgeprägt. Verglichen mit den stationären Spektren in Benzylalkohol ist das globale Maximum der Absorption und Emission in Propylencarbonat leicht blauverschoben.

\footnotetext{
${ }^{3}$ Zur Definition des Begriffs der Dualität: vgl. Kap. 2.2.3
} 


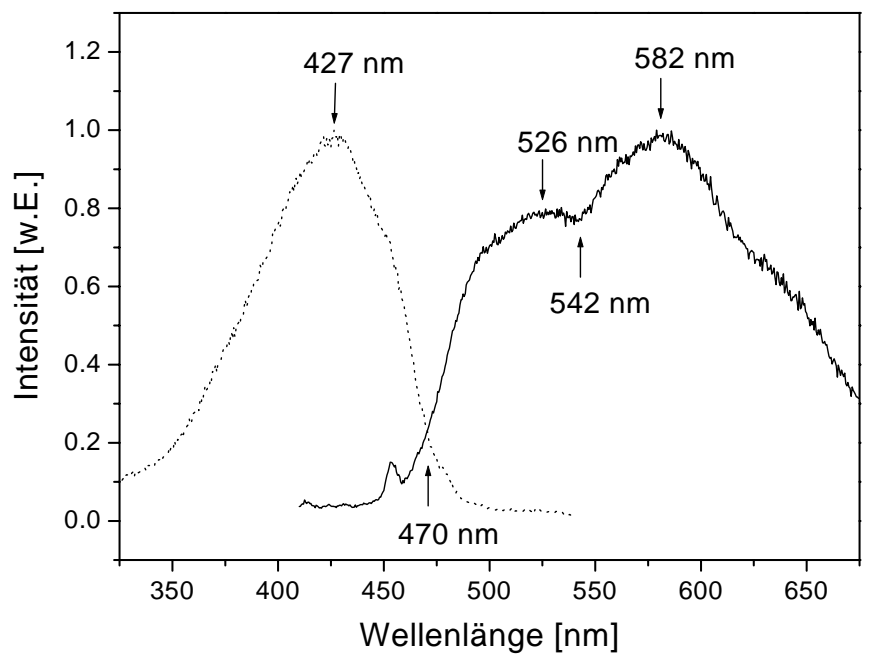

Abbildung 4.7: Stationäres Absorptionsspektrum (gestrichelte Kurve) und Emissionsspektrum (durchgezogene Kurve) nach Anregung bei $400 \mathrm{~nm}$ von 1.8-DHAQ in Propylencarbonat.

\subsubsection{Fluoreszenzdynamik in Propylencarbonat}

Mit Hilfe des FLUC-Experiments wurde die zeitaufgelöste Fluoreszenz von 1.8DHAQ in Propylencarbonat für verschiedene Fluoreszenzwellenlängen zwischen $480 \mathrm{~nm}$ und $620 \mathrm{~nm}$ gemessen. Die Anregung erfolgte bei $400 \mathrm{~nm}$. In Abbildung 4.9 sind repräsentativ fünf der experimentell ermittelten FLUC-Signalkurven zusammen mit den jeweiligen Anpassungskurven abgebildet. Die Anpassung der experimentellen Kurven erfolgte wie in Abschnitt 4.1.3 beschrieben durch die gefaltete Summe aus drei exponentiellen Funktionen. In Tabelle 4.3 sind die auf diese Weise bestimmten Zeitkonstanten zusammengestellt. Im Anhang A ist eine vollständige Liste der ermittelten Anpassungsparameter zu finden.

Das Spektrum von 1.8-DHAQ in Propylencarbonat teilt sich entsprechend der zeitlichen Entwicklung der Fluoreszenzintensität sogar noch eindeutiger als in Benzylalkohol in zwei Hälften. In der einen Hälfte, dem Bereich der kurzwelligen Fluoreszenz, ist nach instantanem Anstieg ein starkes Abklingen der Intensität innerhalb der ersten $200-300 \mathrm{fs}\left(\tau_{\downarrow k u r z}\right)$ zu beobachten, gefolgt von einem langsameren Abfall mit einer Zeitkonstante $\tau_{\downarrow \text { mittel }}$ von wenigen ps. In der anderen Hälfte dagegen, dem Bereich der langwelligen Fluoreszenz, steigt die Intensität nach einem instantanem Anstieg innerhalb der ersten 200-300 fs $\left(\tau_{\uparrow k u r z}\right)$ zunächst stark an, um danach mit einer Zeitkonstante $\tau_{\uparrow m i t t e l}$ von wenigen ps langsamer als zuvor zu zunehmen. Bei Wellenlängen im Übergangsbereich ist wieder wie bei 1.8-DHAQ in Benzylalkohol ein intermediäres Verhalten zu beobachten. 


\begin{tabular}{|c||c|c|c|c|c|}
\hline $\begin{array}{c}\lambda_{\text {Fluoreszenz }} \\
{[\mathrm{nm}]}\end{array}$ & $\begin{array}{c}\tau_{\downarrow \text { lang }} \\
{[\mathrm{ps}]}\end{array}$ & $\begin{array}{c}\tau_{\downarrow \text { mittel }} \\
{[\mathrm{ps}]}\end{array}$ & $\begin{array}{c}\tau_{\downarrow \text { kurz }} \\
{[\mathrm{ps}]}\end{array}$ & $\begin{array}{c}\tau_{\uparrow \text { mittel }} \\
{[\mathrm{ps}]}\end{array}$ & $\begin{array}{c}\tau_{\uparrow \text { kurz }} \\
{[\mathrm{ps}]}\end{array}$ \\
\hline \hline 480 & 350 & 5.0 & 0.312 & - & - \\
\hline 490 & 373 & 2.6 & 0.200 & - & - \\
\hline \hline 500 & 373 & 1.5 & 0.205 & - & - \\
\hline 520 & 309 & - & 0.256 & 15.9 & - \\
\hline 540 & 290 & - & 0.287 & 8.1 & - \\
\hline 560 & 269 & - & - & 8.8 & 0.016 \\
\hline \hline 580 & 276 & - & - & 8.3 & 0.275 \\
\hline 590 & 282 & - & - & 6.0 & 0.181 \\
\hline 600 & 266 & - & - & 6.2 & 0.255 \\
\hline 620 & 269 & - & - & 3.9 & 0.183 \\
\hline
\end{tabular}

Tabelle 4.3: Abklingzeiten $\left(\tau_{\downarrow l a n g}, \tau_{\downarrow \text { mittel }}, \tau_{\downarrow k u r z}\right)$ und Anstiegszeiten ( $\tau_{\uparrow \text { mittel }}$, $\left.\tau_{\uparrow k u r z}\right)$ der Fluoreszenzkurven von 1.8-DHAQ in Propylencarbonat.

In Propylencarbonat stimmen die ermittelten fs-Zeitkonstanten $\tau_{\downarrow k u r z}$ und $\tau_{\uparrow k u r z}$ innerhalb der Genauigkeit des Experiments und der Anpassung (im langwelligen Bereich etwa $\pm 60 \mathrm{fs}$ und im kurzwelligen Bereich etwa $\pm 100 \mathrm{fs}$ ) für alle Wellenlängen überein. Die einzige Ausnahme stellt die Kurve bei $560 \mathrm{~nm}$ dar. Da die Wellenlänge im mittleren Bereich des Spektrums liegt, könnte die hier ermittelte fs-Zeitkonstanten von scheinbar 16 fs durch die Überlagerung von einem $\tau_{\downarrow k u r z}$ und einem $\tau_{\uparrow k u r z}$ in der gleichen Größenordnung zustandegekommen sein. Die mittleren ps-Zeitkonstanten $\tau_{\downarrow \text { mittel }}$ und $\tau_{\uparrow m i t t e l}$ nehmen in beiden Bereichen mit zunehmender Wellenlänge ab.

Die Zeitkonstante $\tau_{\downarrow l a n g}$ von einigen hundert ps ist der Lebensdauer des elektronisch angeregten Zustandes von 1.8-DHAQ zuzuordnen. Diese scheint im Bereich der kurzwelligen Fluoreszenz etwas größer zu sein als im Bereich der langwelligen. Da die Fluoreszenz nur bis zu einer Verzögerung von 100 ps nach der Anregung gemessen wurde, sind die ermittelten langen ps-Zeitkonstanten jedoch mit einer größeren Unsicherheit behaftet.

Die wie in Kapitel 4.1.3 beschrieben rekonstruierte zeitliche Entwicklung des Fluoreszenzspektrums von 1.8-DHAQ in Propylencarbonat ist in Abbildung 4.8 abgebildet.

Auch in Propylencarbonat setzt bei allen beobachteten Wellenlängen eine Fluoreszenzemission innerhalb der zeitlichen Auflösung des FLUC-Experiments ${ }^{4}$ von etwa (100-160) fs instantan mit der Anregung von 1.8-DHAQ ein. Man beobach-

\footnotetext{
${ }^{4}$ Abgeschätzt aus der vollen Halbwertsbreite der angepassten Antwortfunktion.
} 


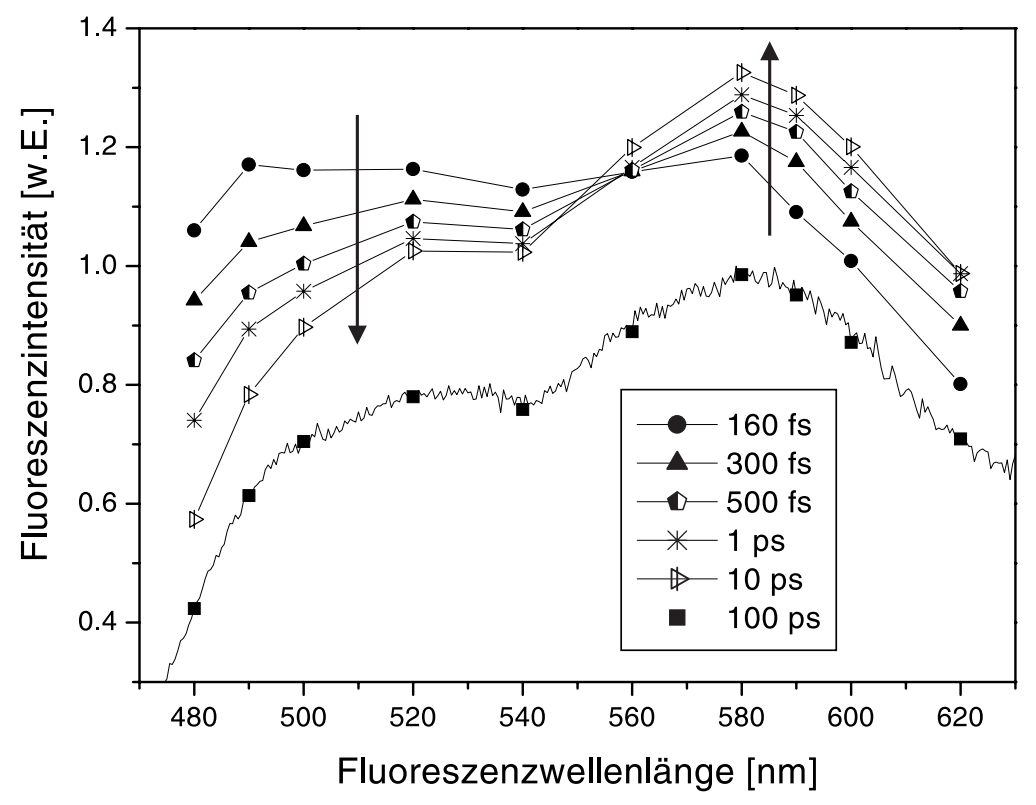

Abbildung 4.8: Zeitliche Entwicklung des Fluoreszenzspektrums von 1.8-DHAQ in Propylencarbonat. Die unterste Kurve kennzeichnet den Verlauf des stationären Spektrums.

tet also bereits 150 fs nach der Anregung eine stark Stokes-verschobene Emission. Die Form des Emissionsspektrums wenige hundert fs nach der Anregung unterscheidet sich merklich von der des stationären Spektrums. Unmittelbar nach der Anregung zeigen die Bereiche der kurzwelligen und langwelligen Fluoreszenz vergleichbare Intensitäten. Mit zunehmender Zeit verlagert sich der Schwerpunkt der Emission zu längeren Wellenlängen hin. Die Entwicklung des zeitabhängigen Spektrums ist wesentlich ausgeprägter als in Benzylalkohol. 

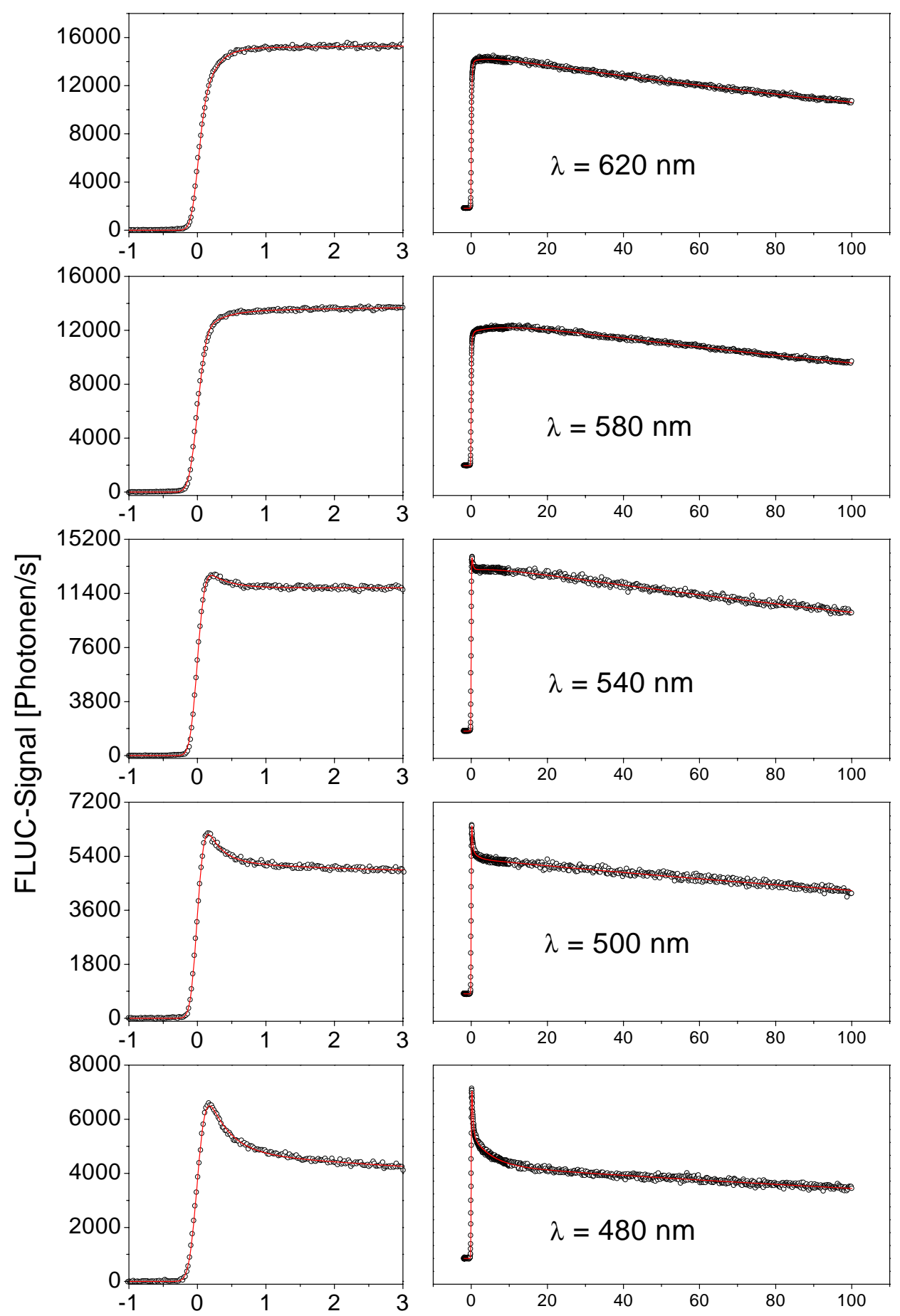

Verzögerungszeit [ps]

Abbildung 4.9: Zeitliche Entwicklung der Fluoreszenzintensität von 1.8-DHAQ in Propylencarbonat nach Anregung bei 400 nm, gemessen für verschiedene Fluoreszenzwellenlängen. Experimentelle Daten sind mit offenen Kreisen, Anpassungskurven mit durchgezogenen Linie gekennzeichnet. 


\subsection{8-DHAQ in Tetrachlorkohlenstoff}

Der Übersichtlichkeit halber wird im folgenden das deuterierte Derivat von 1.8DHAQ, in dem die Hydroxylgruppen-Protonen von 1.8-DHAQ durch Deuterium substituiert wurden, mit 1.8-DDAQ bezeichnet, da es sich bei diesem um 1.8Dideuteriumanthrachinon handelt.

\subsubsection{Einfluss der Deuterierung auf die stationären Spektren}

Abbildung 4.10 zeigt das bei Raumtemperatur aufgenommene stationäre Absorptions- und Emissionsspektrum von 1.8-DHAQ und 1.8-DDAQ in Tetrachlorkohlenstoff. Die Anregung erfolgte bei $395 \mathrm{~nm}$. Der Einfluss der Deuterierung ist deutlich erkennbar. Das Absorptionsspektrum von 1.8-DDAQ in Tetrachlorkohlenstoff zeigt ebenso wenig Struktur wie in den zuvor verwendeten Lösungsmitteln. Es sind lediglich zwei gering ausgeprägte Schultern zu beiden Seiten des Maximums bei etwa $428 \mathrm{~nm}$ zu erkennen. Das Absorptionsspektrum von 1.8DHAQ weist demgegenüber merklich ausgeprägte Schultern zu beiden Seiten des Maximums bei etwa $432 \mathrm{~nm}$ auf. Analoges findet man bei der Betrachtung der Emissionsspektren. Beide Emissionsspektren zeigen eine deutliche duale Struktur. Das globalen Maximum der Emission liegt in der Nähe von $584 \mathrm{~nm}$, und das Nebenmaximum für 1.8-DDAQ bei etwa $500 \mathrm{~nm}$ und für 1.8-DHAQ bei etwa $520 \mathrm{~nm}$. Im Emissionsspektrum von 1.8-DHAQ sind überdies jedoch noch weitere ausgeprägte Schultern neben den beiden Maxima zu erkennen. Im Vergleich zu 1.8-DHAQ sind die Maxima der Absorption und Emission von 1.8-DDAQ leicht blauverschoben.
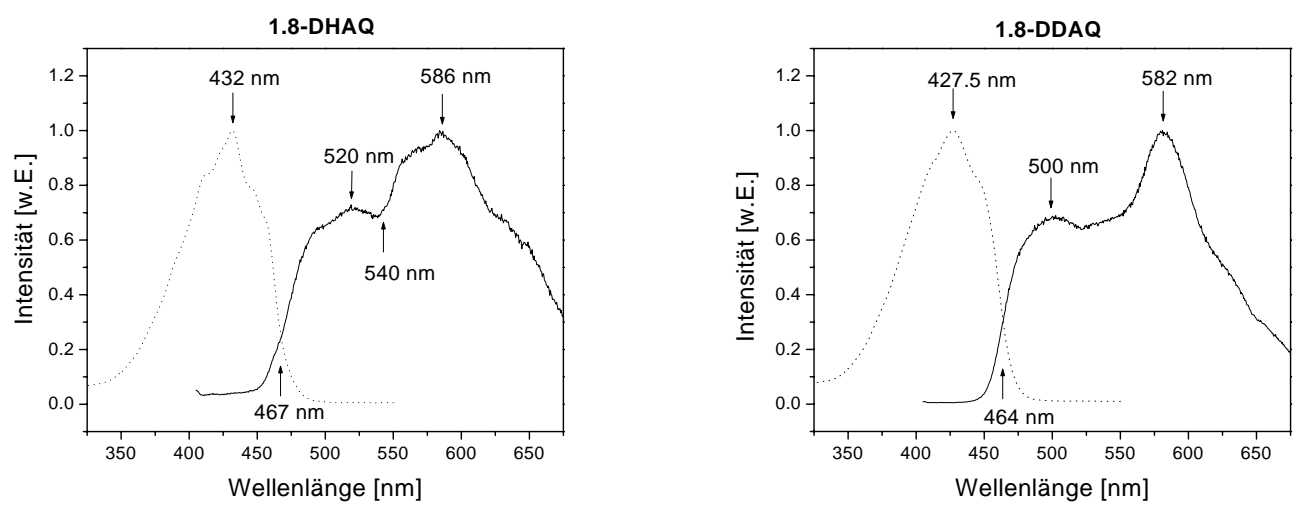

Abbildung 4.10: Stationäres Absorptionsspektrum (gestrichelte Kurve) und Emissionsspektrum (durchgezogene Kurve) nach Anregung bei $395 \mathrm{~nm}$ von 1.8-DHAQ und 1.8-DDAQ in Tetrachlorkohlenstoff. 


\subsubsection{Einfluss der Deuterierung auf die Fluoreszenz- dynamik}

Mit Hilfe des FLUC-Experiments wurde die zeitaufgelöste Fluoreszenz von 1.8DHAQ und 1.8-DDAQ in Tetrachlorkohlenstoff für verschiedene Fluoreszenzwellenlängen zwischen $480 \mathrm{~nm}$ und $630 \mathrm{~nm}$ gemessen. Die Anregung erfolgte bei $395 \mathrm{~nm}$. In den Abbildungen 4.11 bis 4.12 sind repräsentativ acht der experimentell ermittelten FLUC-Signalkurven zusammen mit den jeweiligen Anpassungskurven abgebildet. Die Anpassung der experimentellen Kurven erfolgte so wie in

\begin{tabular}{|c||c|c|c|c|c|}
\hline $\begin{array}{c}\lambda_{\text {Fluoreszenz }} \\
{[\mathrm{nm}]}\end{array}$ & $\begin{array}{c}\tau_{\downarrow l \text { lang }} \\
{[\mathrm{ps}]}\end{array}$ & $\begin{array}{c}\tau_{\downarrow \text { mittel }} \\
{[\mathrm{ps}]}\end{array}$ & $\begin{array}{c}\tau_{\downarrow \text { kurz }} \\
{[\mathrm{ps}]}\end{array}$ & $\begin{array}{c}\tau_{\uparrow \text { mittel }} \\
{[\mathrm{ps}]}\end{array}$ & $\begin{array}{c}\tau_{\uparrow k \text { kurz }} \\
{[\mathrm{ps}]}\end{array}$ \\
\hline \hline 480 & 196 & 6.2 & 0.248 & - & - \\
\hline 490 & 165 & 1.6 & 0.118 & - & - \\
\hline 500 & 210 & 8.2 & 0.218 & - & - \\
\hline 520 & 173 & 3.6 & 0.234 & - & - \\
\hline 540 & 173 & 7.2 & 0.231 & - & - \\
\hline \hline 560 & 140 & - & 0.633 & 16.1 & - \\
\hline \hline 585 & 177 & - & - & 19.7 & 0.135 \\
\hline 605 & 181 & - & - & 12.7 & 0.248 \\
\hline 630 & 197 & - & - & 8.7 & 0.178 \\
\hline
\end{tabular}

Tabelle 4.4: Abklingzeiten ( $\left.\tau_{\downarrow l a n g}, \tau_{\downarrow \text { mittel }}, \tau_{\downarrow k u r z}\right)$ und Anstiegszeiten ( $\tau_{\uparrow \text { mittel }}$, $\left.\tau_{\uparrow k u r z}\right)$ der Fluoreszenzkurven von 1.8-DHAQ in Tetrachlorkohlenstoff.

\begin{tabular}{|c||c|c|c|c|c|}
\hline $\begin{array}{c}\lambda_{\text {Fluoreszenz }} \\
{[\mathrm{nm}]}\end{array}$ & $\begin{array}{c}\tau_{\downarrow \text { lang }} \\
{[\mathrm{ps}]}\end{array}$ & $\begin{array}{c}\tau_{\downarrow \text { mittel }} \\
{[\mathrm{ps}]}\end{array}$ & $\begin{array}{c}\tau_{\downarrow \text { kurz }} \\
{[\mathrm{ps}]}\end{array}$ & $\begin{array}{c}\tau_{\uparrow \text { mittel }} \\
{[\mathrm{ps}]}\end{array}$ & $\begin{array}{c}\tau_{\uparrow k \text { kurz }} \\
{[\mathrm{ps}]}\end{array}$ \\
\hline \hline 480 & 163 & 8.2 & 0.381 & - & - \\
\hline 490 & 111 & 2.3 & 0.167 & - & - \\
\hline 500 & 200 & 7.8 & 0.255 & - & - \\
\hline \hline 520 & 172 & 1.2 & 0.077 & - & - \\
\hline 540 & 222 & 0.62 & - & - & - \\
\hline 560 & 230 & 0.35 & - & - & - \\
\hline \hline 585 & 224 & - & - & 12.9 & 0.078 \\
\hline 605 & 257 & - & - & - & 0.112 \\
\hline 630 & 238 & - & - & 19.3 & 0.476 \\
\hline
\end{tabular}

Tabelle 4.5: Abklingzeiten ( $\left.\tau_{\downarrow l a n g}, \tau_{\downarrow \text { mittel }}, \tau_{\downarrow 3 k u r z}\right)$ und Anstiegszeiten $\left(\tau_{\uparrow \text { mittel }}\right.$, $\left.\tau_{\uparrow k u r z}\right)$ der Fluoreszenzkurven von 1.8-DDAQ in Tetrachlorkohlenstoff. 

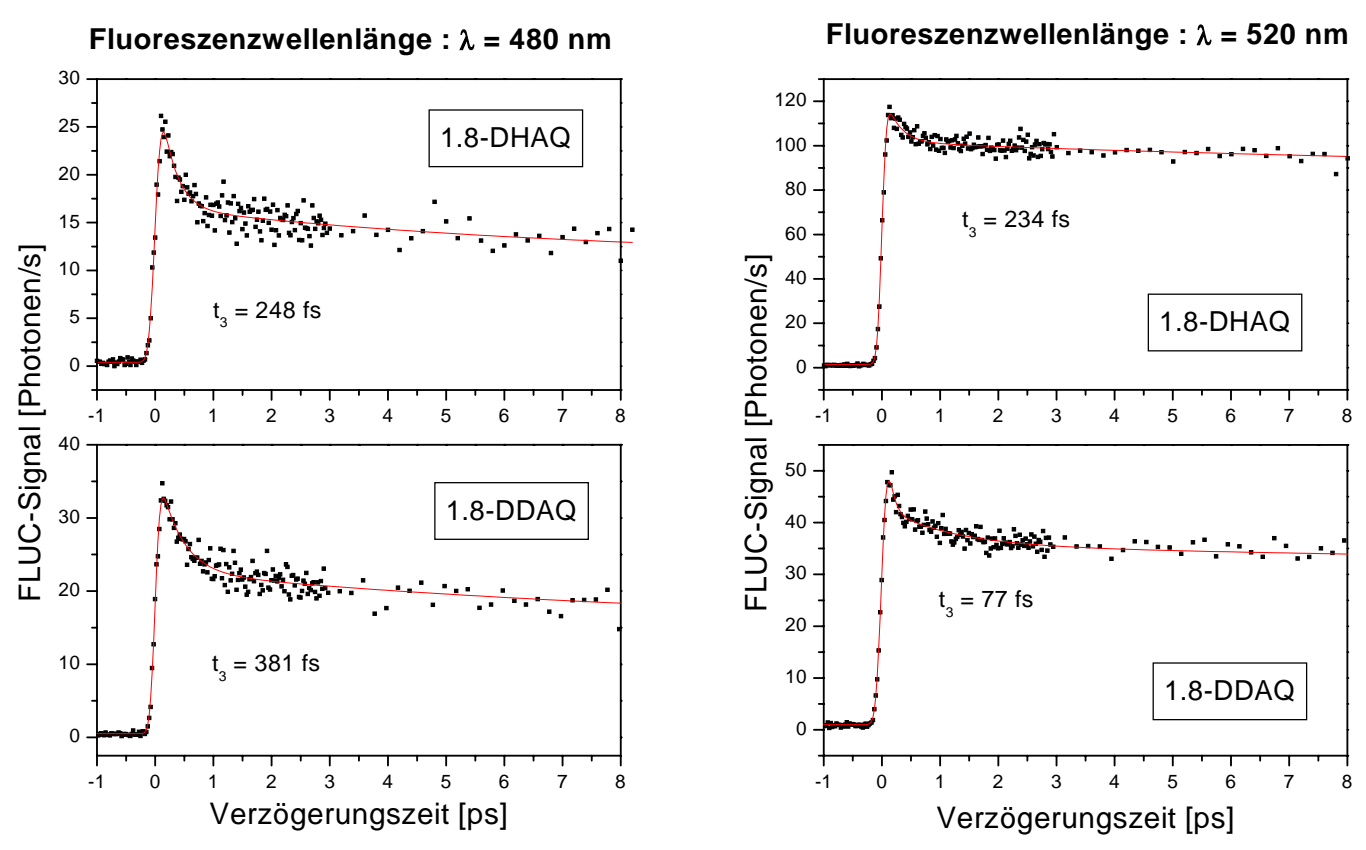

Abbildung 4.11: Zeitliche Entwicklung der Fluoreszenzintensität von 1.8-DHAQ und 1.8-DDAQ in $C C l_{4}$ nach Anregung bei $395 \mathrm{~nm}$. Experimentelle Daten sind mit offenen Kreisen, Anpassungskurven mit durchgezogenen Linie gekennzeichnet.

Kapitel 4.1.3 beschrieben durch gefaltete multiexponentielle Funktionen. In Tabelle 4.4 sind die auf diese Weise bestimmten Zeitkonstanten zusammengestellt. Im Anhang A ist eine vollständige Liste der ermittelten Anpassungsparameter zu finden.

Wie in den Abbildungen 4.11 und 4.12 zu erkennen, ist das Signal-zu-RauschVerhältnis mit 4:1 im Bereich kürzerer Fluoreszenzwellenlängen bzw. 16:1 im Bereich längerer Fluoreszenzwellenlängen bei diesen Messungen trotz langer Messzeiten (vgl. Kap. 3.2.4) schlechter als bei den vorangegangenen FLUC-Messungen in Benzylalkohol und Proplylencarbonat (16:1 im kurzwelligen Bereich bis 48:1 im langwelligen Bereich). Dies liegt daran, dass aufgrund des leicht flüchtigen Lösungsmittels und des deuterierten Derivates an einer geschlossenen Rotationszelle gemessen werden musste. Die Probe konnte daher nur mit geringer Intensität angeregt werden, damit sie sich nicht zersetzte. Dies hat zur Folge das die ermittelten Zeitkonstanten mit einem relativ großen Unsicherheitsfaktor behaftet sind, und daher nur für qualitative Aussagen geeignet sind.

Ebenso wie bei den bisher betrachteten Lösungsmitteln teilt sich das Spektrum 

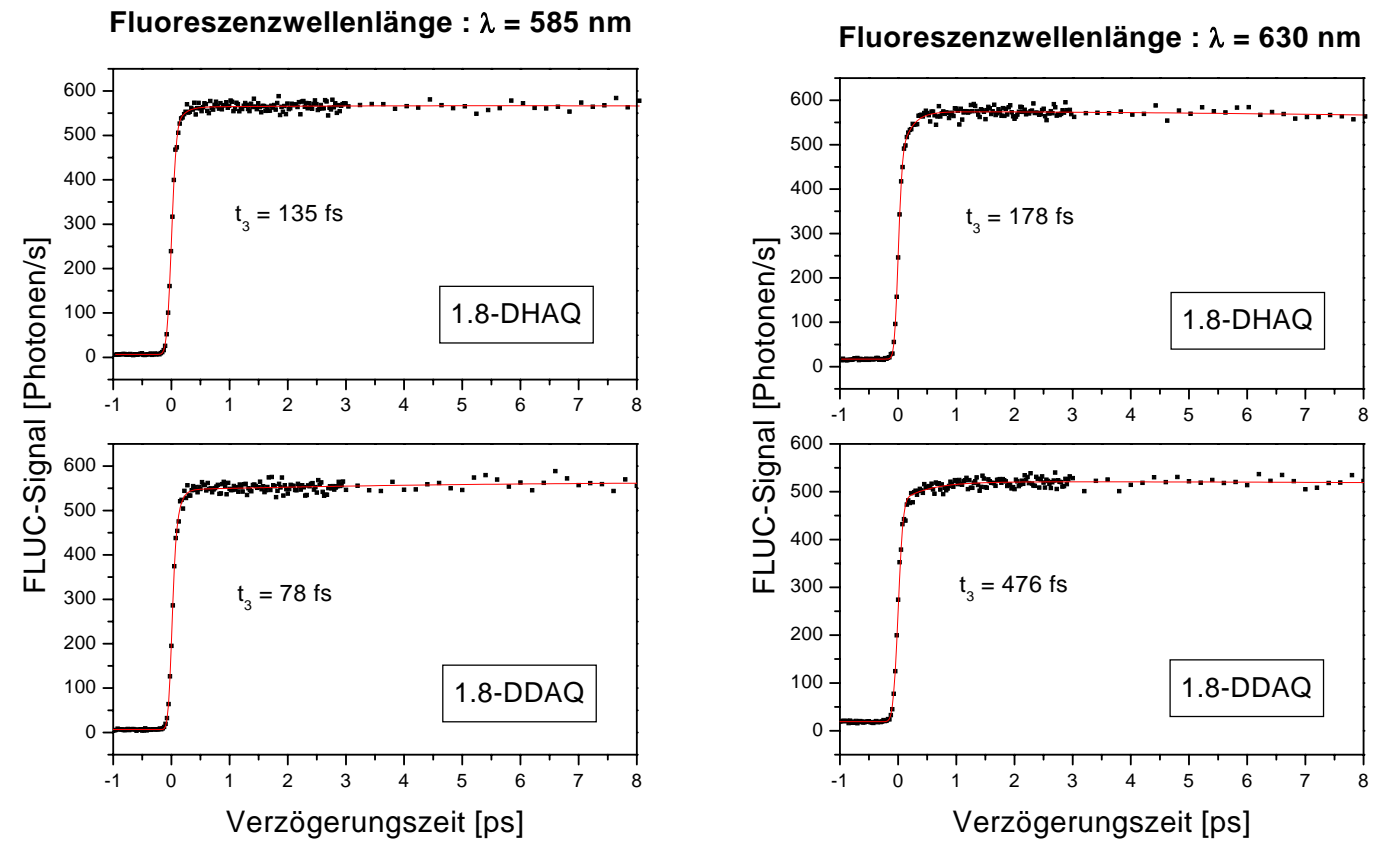

Abbildung 4.12: Zeitliche Entwicklung der Fluoreszenzintensität von 1.8-DHAQ und 1.8-DDAQ in $\mathrm{CCl}_{4}$ nach Anregung bei $395 \mathrm{~nm}$. Experimentelle Daten sind mit Punkten, Anpassungskurven mit durchgezogenen Linie gekennzeichnet.

von 1.8-DHAQ in Tetrachlorkohlenstoff entsprechend der zeitlichen Entwicklung der Fluoreszenzintensität in den Bereich der kurzwelligen Fluoreszenz, in dem ein starkes Abklingen der Intensität auf Zeitskalen weniger hundert fs und weniger ps zu beobachten ist, und den Bereich der langwelligen Fluoreszenz, in dem die Intensität auf diesen Zeitskalen ansteigt. Auch wenn es den Anschein hat, dass die schnellste Zeitkonstante $\tau_{\downarrow k u r z}$ im Wellenlängenbereich von $480 \mathrm{~nm}$ bis $520 \mathrm{~nm}$ für 1.8-DDAQ etwas größer ist als für 1.8-DHAQ, kann innerhalb der Fehlergrenzen der Zeitkonstanten kein Isotopeneffekt festgestellt werden. Die Lebensdauer des angeregten Zustandes scheint für die deuterierte Verbindung etwas größer $\mathrm{zu}$ sein, doch auch diese Beobachtung ist angesichts der Ungenauigkeit bei der Bestimmung der Parameter, da die Fluoreszenz nur bis zu einem Zeitpunkt von 100 ps nach der Anregung gemessen wurde und überdies das Signal-zu-RauschVerhältnis relativ schlecht ist, nicht sicher.

Die aus den Signalkurven ermittelte Rekonstruktion (vgl. Kap. 3.2.4) der zeitlichen Entwicklung des Fluoreszenzspektrums von 1.8-DHAQ und 1.8-DDAQ in Tetrachlorkohlenstoff ist in Abbildung 4.13 abgebildet.

Auch in Tetrachlorkohlenstoff setzt bei allen beobachteten Wellenlängen eine Fluoreszenzemission innerhalb der zeitlichen Auflösung des FLUC-Experiments 

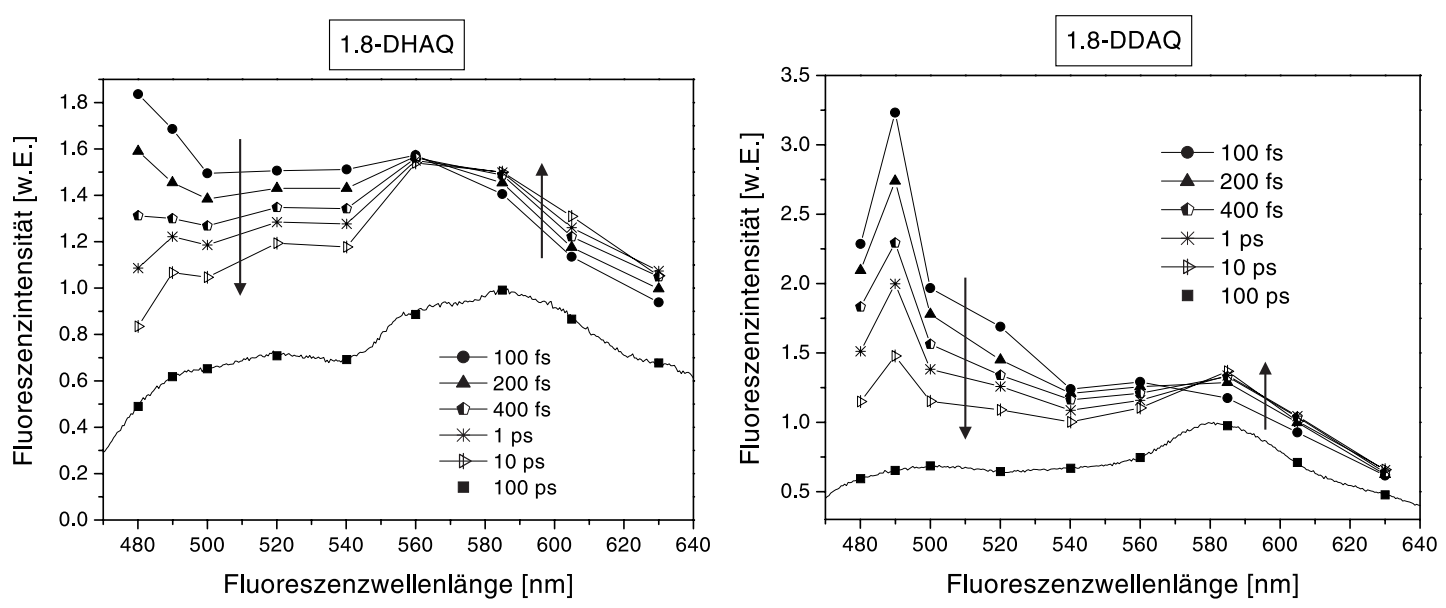

Abbildung 4.13: Zeitliche Entwicklung des Fluoreszenzspektrums von 1.8-DHAQ (links) und 1.8-DDAQ (rechts) in Tetrachlorkohlenstoff. Die untersten Kurven kennzeichnen den Verlauf der stationären Spektren.

instantan mit der Anregung der Probe ein. Betrachtet man zu einem festen Zeitpunkt wenige hundert fs nach der Anregung die relative Fluoreszenzintensität bei verschiedenen Wellenlängen, scheint die relative Intensität zu kleineren Wellenlängen ( $500 \mathrm{~nm} \rightarrow 480 \mathrm{~nm}$ ) hinzuzunehmen. Da jedoch keine Messungen bei Fluoreszenzwellenlängen kleiner als $480 \mathrm{~nm}$ durchgeführt wurden und die existierenden Messungen aufgrund des schlechten Signal-zu-Rausch-Verhältnisses keine sichere Anpassung ermöglichen, sollte diese Beobachtung nicht überbewertet werden. Letzteres gilt auch für den herausstechenden Peak bei $490 \mathrm{~nm}$ im zeitabhängigen Spektrum von 1.8-DDAQ. Um seine Relevanz zu testen, müssten weitere Messungen bei benachbarten Wellenlängen durchgeführt werden. Die Entwicklung des zeitabhängigen Fluoreszenzspektrums ist in Tetrachlorkohlenstoff noch ausgeprägter als in Benzylalkohol oder Propylencarbonat. Die Form des Emissionsspektrums unmittelbar nach der Anregung unterscheidet sich merklich von der des stationären Spektrums. Während der ersten hundert fs ist die Intensität im Bereich der kurzwelligen Fluoreszenz vergleichbar oder sogar größer als die Intensität im Bereich der langwelligen Fluoreszenz. Mit zunehmender Zeit verlagert sich der Schwerpunkt der Emission zu längeren Wellenlängen hin. Die Dynamik ist dabei im kurzwelligen Bereich des Spektrums wesentlich ausgeprägter. Bei 1.8-DHAQ ist dies noch deutlicher als bei 1.8-DHAQ. 


\section{Kapitel 5}

\section{Diskussion}

Zunächst folgt eine Kurzfassung des in Kapitel 2.2.3 ausführlich erläuterten, bisherigen Modells der Photochemie von 1.8-DHAQ. Wie beschrieben wurde, folgerten Smulevich et al. und Gillispie et al. aus der Analyse ihrer Ergebnisse frequenzaufgelöster Messungen (Stokesverschiebung, duale Absorption, duale Emission, $\nu(\mathrm{OH})$-Progression auf dem $\mathrm{S}_{0} \rightarrow \mathrm{S}_{1}$ Übergang, Intensität und Frequenz der Moden im wasserstoffgebundenen Ring), dass der wasserstoffgebundene quasiaromatische $\mathrm{C}_{1 / 8^{-}} \mathrm{O}-\mathrm{H} \cdots \mathrm{O}=\mathrm{C}_{9}$ Ring am $\pi \rightarrow \pi^{*}$ Übergang vom Grundzustand in den ersten elektronisch angeregten Zustand von 1.8-DHAQ beteiligt ist. Ferner entnahmen sie ihren Analysen, dass der Abstand $\mathrm{R}(\mathrm{O} \cdots \mathrm{O})$ zwischen den Sauerstoffatomen der Carbonyl- und Hydroxylgruppe bei der Anregung um etwa $0.16 \AA$ verringert, die Wasserstoffbrückenbindung gestärkt und hierdurch ein Protontransfer im angeregten Zustand auslöst wird.

Nach den Analysen von Smulevich et al. lassen sich die stationären spektroskopischen Eigenschaften von 1.8-DHAQ gut beschreiben durch die Annahme eines asymmetrischen Lippincott-Schröder-Doppelminimum-Potentials entlang der $\mathrm{r}(\mathrm{OH})$-Koordinate im elektronischen Grundzustand, dessen energetisch tiefer liegendes Minimum die Normalform des Moleküles repräsentiert und dessen zweites Minimum dem Protontransfer-Tautomer zuzuordnen ist. Das Potential im angeregten Zustand sollte demgegenüber eine leicht asymmetrische DoppelminimumForm inverser Symmetrie bezüglich des Potentialverlaufs im Grundzustand aufweisen und eine so niedrige Energiebarriere besitzen, dass sich das Nullpunktniveau im energetisch tieferliegenden Minimum knapp unterhalb dieser befindet, während im zweiten Minimum kein quantenmechanisch gebundener Zustand mehr existieren kann (vgl. Abb. 5.1). Für höher vibronisch angeregte Zustände kann das Potential entlang der $\mathrm{r}(\mathrm{OH})$-Koordinate daher als näherungsweise harmonisch betrachtet werden, mit einem globalen Minimum, dessen Lage sich zwischen den beiden Minima des Grundzustandes befindet. Letzteres hat zur Folge, dass die Wellenfunktionen in vibronisch angeregten Zuständen von $\mathrm{S}_{1}$ über beide Minima des Grundzustandes delokalisiert ist. Da sich das Smulevich-Modell 
sowohl für die Gasphase als auch für die Messungen in der Matrix sehr bewährt hat, wird es in der folgenden Interpretation auch als Grundlage für ein Modell zur Erklärung der Beobachtungen in der flüssigen Phase herangezogen.

Bevor nun diskutiert wird ob und wie sich die in Pump-Probe- und FLUCExperimenten gemachten Beobachtungen in das bestehende Modell für den Protontransfer in 1.8-DHAQ einfügen lassen oder in welcher Hinsicht es einer Erweiterung bedarf, soll zunächst noch ein zusammenfassender Überblick über die experimentellen Ergebnisse in dieser Arbeit gegeben werden.

Wie die zeitaufgelösten Fluoreszenzmessungen zeigen setzt die spontane Emission von 1.8-DHAQ unabhängig vom Lösungsmittel innerhalb der Zeitauflösung des FLUC-Experiments von etwa (100-160) fs quasi instantan mit der Anregung ein und liegt bereits zu diesem Zeitpunkt im Wellenlängenbereich der stark Stokes-verschobenen stationären Emission. Hinsichtlich der Fluoreszenzdynamik besitzt 1.8-DHAQ in allen drei untersuchten Lösungsmitteln ein qualitativ ähnliches Verhalten. Analog zu der Dualität des stationären Emissionsspektrums findet man in Bezug auf die zeitliche Entwicklung der Fluoreszenz (gemäß der FLUC-Kurven) in allen Lösungsmitteln zwei spektrale Bereiche entgegengesetzter Dynamik (vgl. Abb. 4.6, 4.8, 4.13). Im Wellenlängenbereich der KWE ("kürzere Wellenlängen des stationären Emissionsspektrums") findet man ein stärkeres Abklingen der Fluoreszenzintensität, innerhalb der ersten $\tau_{\downarrow k u r z} \simeq(200-450)$ fs und ein schwächeres Abklingen innerhalb der folgenden $\tau_{\downarrow \text { mittel }} \simeq(2-25)$ ps. Tendenziell scheint $\tau_{\downarrow k u r z}$ im Bereich der KWE in Benzylalkohol etwas größer zu sein als in Propylencarbonat und Tetrachlorkohlenstoff. Im Wellenlängenbereich der LWE ("längere Wellenlängen des stationären Emissionsspektrums") hingegen zeigen die FLUC-Signale ein stärkeres Ansteigen der Fluoreszenzintensität, innerhalb der ersten $\tau_{\uparrow k u r z} \simeq(100-300)$ fs und ein schwächeres Ansteigen innerhalb der folgenden $\tau_{\uparrow \text { mittel }} \simeq(1-20)$ ps. Nur in Benzylalkohol ist im LWE-Bereich noch eine zusätzliche auf der Zeitskala von 5-35 ps abfallende Fluoreszenzkomponente nötig, um die experimentellen Daten anzupassen. Der Einfluß des Lösungsmittels macht sich vor allem in der unterschiedlich ausgeprägten zeitlichen Entwicklung des Fluoreszenzspektrums innerhalb der ersten wenigen hundert fs bemerkbar. Während das spontane Emissionsspektrum von 1.8-DHAQ in Benzylalkohol bereits 150 fs nach der Anregung große Ähnlichkeit mit dem stationären Emissionsspektrum aufweist, unterscheiden sich die spontanen Emissionsspektren in Propylencarbonat und Tetrachlorkohlenstoff zu diesem Zeitpunkt noch merklich vom stationären Fall. Zwar liegt die Fluoreszenz bereits im stark Stokes-verschobenen Bereich, die Emission im KWE-Bereich ist aber deutlich erhöht, und der Schwerpunkt der spontanen Emission ist daher noch leicht blauverschoben.

Der Einfluss des Lösungsmittels auf die stationären spektroskopischen Eigenschaften von 1.8-DHAQ äußert sich in einer von Benzylalkohol über Propylencarbonat 
zu Tetrachlorkohlenstoff zunehmenden Strukturierung der eher formlosen stationären Absorptionsspektren und einer stärker ausgeprägten Dualität der stationären Emissionsspektren.

Hinsichtlich der Abhängigkeit der Zeitkonstanten von der Wellenlänge kann bemerkt werden, dass $\tau_{\downarrow k u r z}$ und $\tau_{\uparrow k u r z}$ jeweils im Rahmen der Fehlertoleranzen der experimentellen Daten konstant sind, während die $\tau_{\downarrow \text { mittel }}$ und $\tau_{\uparrow m i t t e l}$ demgegenüber eine Abhängigkeit von der Wellenlänge aufweisen.

Die Deuterierung der Hydroxylgruppen von 1.8-DHAQ, die sich deutlich auf die stationären Spektren auswirkt, hat in Bezug auf die Fluoreszenzdynamik keine eindeutige Tendenz der Zeitkonstanten zur Folge. Zwar scheint $\tau_{\downarrow k u r z}$ im Wellenlängenbereich von 480-500 nm im Falle der Deuterierung etwas größer zu werden. Unter Berücksichtigung der Fehlergrenzen der Anpassung ergibt sich jedoch keine signifikante Abhängigkeit der Dynamik.

Bei der Betrachtung des transienten Transmissionsverhaltens des angeregten 1.8DHAQ (gemäß der Pump-Probe-Kurven) findet man analog zu der Aufteilung des Spektrums in Hinblick auf die Fluoreszenzdynamik ebenfalls zwei unterschiedliche Bereiche. Im Wellenlängenbereich der KWE zeigen die Pump-Probe-Signale eine transiente Absorption aus $\mathrm{S}_{1}$ in höhere elektronisch angeregte Zustände $\mathrm{S}_{n}$, im Wellenlängenbereich der LWE hingegen eine stimulierte Emission aus $S_{1}$ in den elektronischen Grundzustand. Nach Vöhringer et al. [46] entsteht ebenso wie bei der Fluoreszenz auch der Großteil des Pump-Probe-Signals bei allen beobachteten Wellenlängen innerhalb der ersten $100 \mathrm{fs}$, allerdings wachsen transiente Absorption und stimulierte Emission danach mit derselben Zeitkonstante von etwa $300 \mathrm{fs}$ weiter an [46]. Die Pump-Probe-Signale weisen überdies eine schwach gedämpfte $(\tau \approx 600-900 \mathrm{fs})$, periodische Modulation der Intensität auf, mit einer besonders starken Oszillation bei $(207-215) \mathrm{cm}^{-1}$ und einer die Grenzen der Nachweisempfindlichkeit erreichenden bei $(330-350) \mathrm{cm}^{-1}$.

\subsection{Ursprung der Emission}

Man ist zunächst geneigt die beiden spektralen Bereiche mit so unterschiedlichen spektroskopischen Eigenschaften von 1.8-DHAQ mit der lokal angeregten Normalform und dem Protontransfer-Tautomer im elektronisch angeregten $\mathrm{Zu}$ stand in Verbindung zu bringen. Da die beobachtete Fluoreszenz (FLUC-Signal) und auch die stimulierte Emission (Pump-Probe-Signal) jedoch bereits unmittelbar nach der Anregung stark Stokes-verschoben ist, müssten bereits innerhalb der ersten hundert fs größere strukturelle Änderungen im Molekül stattgefunden haben. Um die Beobachtung der beiden Bereiche zu erklären, ist es auch 
Eindimensionales-LS-Potential

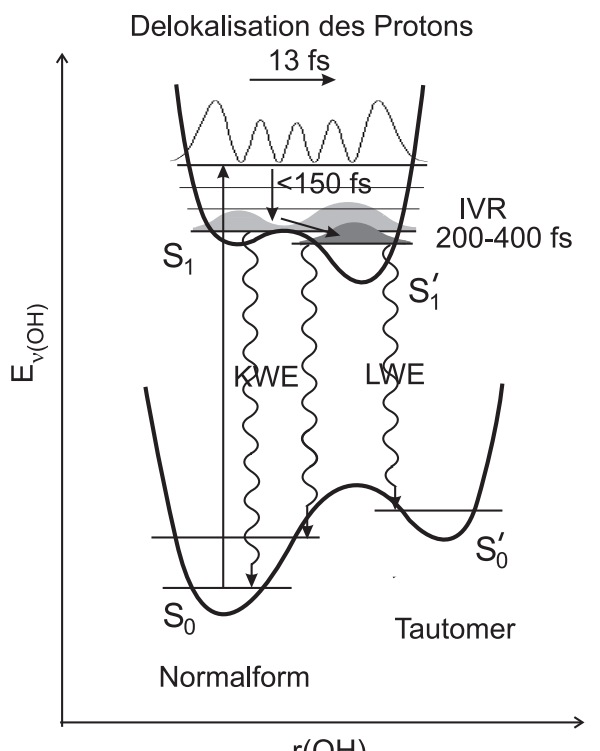

Multidimenionaler Potentialverlauf

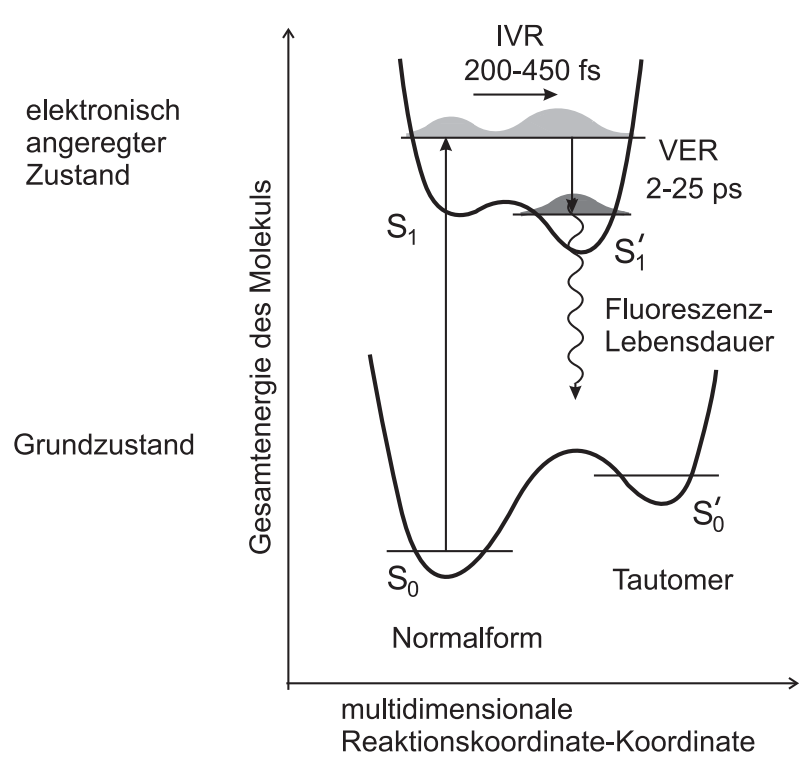

Abbildung 5.1: Modell des Protontransfers im ersten elektronisch angeregten Zustand von 1.8-DHAQ.

gar nicht nötig, die Existenz zweier verschiedener chemischer Spezies im ersten elektronisch angeregten Zustand anzunehmen. Vielmehr lässt sich eine solche Beobachtung ebenso mit der Annahme einer einzigen Wellenfunktion im ersten elektronisch angeregten Zustand erklären, die sich über die beiden Potentialminima des Grundzustandes erstreckt, wie von Smulevich et al. vorgeschlagen. Die beiden spektralen Bereiche im zeitabhängigen Fluoreszenzspektrum spiegeln in diesem Fall die zeitliche Entwicklung des Franck-Condon-Überlapp (FC-Überlapp) der über beide Potentialminima des Grundzustandes delokalisierten Wellenfunktion im vibronisch angeregten Zustand mit der Wellenfunktion im Grundzustand der Normalform $\mathrm{S}_{0}$ und der tautomeren Form $\mathrm{S}_{0}^{\prime}$ des Moleküls wieder (vgl. Abb. 5.1). Entsprechendes gilt für das zeitabhängige Transmissionsspektrum. Für dessen Eigenschaften spielt allerdings auch zusätzlich die Entwicklung des FC-Überlapp der Wellenfunktion im $\mathrm{S}_{1}$-Zustand mit den Wellenfunktionen in höhere elektronisch angeregte Zustände $\mathrm{S}_{n}$ eine Rolle. Die Existenz einer einzigen delokalisierten Wellenfunktion im vibronisch angeregten Zustand wird auch durch Tahara et al. unterstützt, der in zeitaufgelösten FLUC-Messungen an 1.8-DHAQ in Hexan eine gleiche Fluoreszenzlebensdauer in beiden Bereichen der Emission beobachtet hat [47]. Die Frage, ob es sich bei der entlang der r(OH)-Koordinate delokalisierten Wellenfunktion um eine Mischform aus $\mathrm{S}_{1}$ - und $\mathrm{S}_{1}^{\prime}$-Zustand handelt oder um einen reinen $\mathrm{S}_{1}^{\prime}$-Zustand, wird später noch diskutiert werden. 


\subsection{Instantane Stokesverschiebung}

In den durchgeführten Experimenten wurde das Molekül bei 395-400 nm angeregt, d.h. nach den Analysen von Smulevich et al. mit einem Überschuss an Schwingungsenergie in der Größenordnung von $3400 \mathrm{~cm}^{-1}$ oberhalb des rein elektronischen $\mathrm{S}_{0} \rightarrow \mathrm{S}_{1}$ Übergangs der Normalform und etwa $4100 \mathrm{~cm}^{-1}$ oberhalb des rein elektronischen $\mathrm{S}_{0} \rightarrow \mathrm{S}_{1}^{\prime}$ Übergangs in die tautomere Form des Moleküls. Nach Smulevich et al. findet nach Anregung bei $390 \mathrm{~nm}$ der Übergang $\mathrm{S}_{0} \rightarrow \mathrm{S}_{1}+3 \nu(\mathrm{OH})$ statt und man befindet sich weit oberhalb einer eventuell vorhandenen kleinen Potentialbarriere in einem Bereich, in dem $S_{1^{-}}$und $S_{1}^{\prime}$-Zustand in dem LSPotentialbild nicht mehr voneinander zu trennen sind. Da die Schwingungsdauer der $\nu(\mathrm{OH})$-Mode im angeregten Zustand etwa 13 fs beträgt (entsprechend einer Vibrationsfrequenz von $1300 \mathrm{~cm}^{-1}$ ), sollte die Wellenfunktion bereits innerhalb der Dauer des Anregungspulses von etwa 30 fs entlang der $\mathrm{r}(\mathrm{OH})$-Koordinate delokalisiert sein. Das allein reicht aber nicht aus als Grund für die beobachtete starke Stokesverschiebung der Emission (vgl. Abb. 5.1). Eine mögliche Erklärung ist die Relaxation innerhalb der $\nu(\mathrm{OH})$-Schwingungsmode durch sehr schnelle IVRProzesse $^{1}$, die im wesentlichen zu einer Population der beiden untersten Zustände im LS-Potential führen würde, also insbesondere zu einer starken Besetzung des $\mathrm{S}_{1}^{\prime}$-Zustands. Innerhalb der ersten hundert fs sollte also der Protontransfer weitgehend abgeschlossen sein. Eine solche schnelle Relaxation könnte durch eine starke anharmonische Kopplung der $\nu(\mathrm{OH})$-Schwingungsmode an andere Moden begünstigt werden, die den Energiefluß in diese ermöglicht. Das solche Kopplungen existieren müssten wird später in der Diskussion gezeigt werden. Eine andere mögliche Erklärung für die nahezu instantan stark Stokes-verschobene Emission basiert auf Berechnungen durch Nagaoka et al. (vgl. Kap. 2.2.3). Hiernach findet nach der Anregung von 1.8-DHAQ eine Delokalisierung der beiden ungepaarten Elektronen des angeregten $\pi \pi^{*}$-Zustands statt. Diese bedingt, dass das Molekül eine stark veränderte Potentiallandschaft vorfindet, innerhalb derer es beginnt sich zu entwickeln. Die Anregungsenergie würde auf diese Weise auf verschiedene Schwingungsfreiheitsgrade verteilt werden, bezüglich derer sich das elektronisch umstrukturierte Molekül unter Begünstigung der Relaxation der $\nu(\mathrm{OH})$-Mode entwickelt. Nach beiden Erklärungsmodellen kann die starke instantane Stokesverschiebung also der Relaxation der $\nu(\mathrm{OH})$-Schwingungsmode zugeordnet werden. Welche der Möglichkeiten hier wahrscheinlicher ist, wird deutlicher, wenn der Ursprung der Oszillationen im Pump-Probe-Signal besprochen wurde.

\footnotetext{
${ }^{1} \mathrm{IVR}=$ Intramolecular Vibrational Redistribution
} 


\subsection{Zuordnung der Zeitkonstanten}

Es folgen einige prinzipielle Überlegungen zum möglichen Ursprung der verschiedenen Zeitkonstanten. Vor der Anregung befindet sich das Molekül im elektronischen Grundzustand der Normalform, mit einer thermischen Besetzung der Schwingungsmoden (d.h. $\left.\mathrm{n}_{\nu(\mathrm{OH})}=0\right)$. Bei einer vibronischen Anregung des Moleküls mit einem großen Überschuss an Schwingungsenergie werden nur solche Schwingungsfreiheitsgrade des angeregten Zustandes erreicht die einen endlich großen FC-Überlapp mit dem elektronischen Grundzustand der Normalform aufweisen. Durch anharmonische Wechselwirkungen oder Fermi-Resonanzen sind jedoch weitere Schwingungsfreiheitsgrade an die direkt durch Anregung besetzten Zustände gekoppelt. Zu einer zeitlichen Entwicklung des FC-Überlapp kann daher nicht nur die strukturelle Umwandlung des Moleküls infolge des Transfers eines Protons führen, sondern auch die intramolekulare Umverteilung der kurz nach der Anregung in wenigen Freiheitsgraden lokalisierten Vibrationsenergie auf diese zusätzlichen Schwingungsfreiheitsgrade (IVR). Diese Umverteilung führt zu einer statistischen Besetzung aller zugänglichen, angeregten Zustände gemäß einer Boltzmannverteilung. Eine weitere Ursache für eine zeitliche Entwicklung des FC-Überlapp ist die abnehmende Population in höheren vibronisch angeregten Zuständen infolge der Energierelaxation der Probenmoleküle hauptsächlich durch Stöße mit dem Lösungsmittel (VER) ${ }^{2}$ zugunsten energetisch tiefer liegender Vibrationszustände (vibrational cooling). Wichtig ist, dass hierbei nicht nur der eindimensionale FC-Überlapp entlang der $\mathrm{r}(\mathrm{OH})$-Schwingungskoordinate betrachtet werden muss, sondern auch der FC-Überlapp zwischen dem angeregten Zustand und dem Grundzustand entlang anderer Schwingungskoordinaten, die infolge von IVR besetzt wurden. Es wird somit deutlich, dass das eindimensionale Bild des Protontransfers entlang der $\mathrm{r}(\mathrm{OH})$-Koordinate des LS-Potentials nicht ausreicht für eine angemessene Beschreibung der realen Vorgänge.

In Lösungen wurden für große Moleküle (mehr als 20 Atome) im ersten angeregten Singulettzustand Zeitkonstanten unter hundert fs für IVR-Prozesse zwischen resonanten Schwingungszuständen und von mehreren hundert fs zwischen nicht resonanten gefunden. Die Zeitskalen für VER liegen dagegen bei (1-50) ps [61]. Da die kürzesten im Experiment aufösbaren Zeitkonstanten $\tau_{\downarrow k u r z}$ und $\tau_{\uparrow k u r z}$ von $\simeq(100-450)$ fs im Rahmen der Fehlertoleranzen der experimentellen Daten relativ konstant $\operatorname{sind}^{3}$, liegt es nahe diese mit einer Änderung des FC-Überlapps infolge von IVR-Prozess zwischen wenigen mit der $\nu(\mathrm{OH})$-Mode gekoppelten Schwingungsfreiheitsgraden in Verbindung zu bringen, die zu einer abnehmenden Besetzung im $\mathrm{S}_{1}$-Zustand führt zugunsten des $\mathrm{S}_{1}^{\prime}$ Zustand führt (vgl. Abb. 5.1). Die mittleren Zeitkonstanten $\tau_{\downarrow \text { mittel }}$ und $\tau_{\uparrow m i t t e l}$ von $\simeq(1-25)$ ps die eine stärke-

\footnotetext{
${ }^{2} \mathrm{VER}=$ Vibrational Energy Relaxation

${ }^{3}$ Hiervon ausgenommen ist der Übergangsbereich.
} 
re Abhängigkeit von der Wellenlänge aufweisen, sind dagegen hauptsächlich als Folge von VER zu betrachten.

\subsection{Lösungsmittelabhängigkeit}

Welche Informationen sind in der Lösungsmittelabhängigkeit der Signale enthalten? Es liegt nahe, hierüber Aussagen zu gewinnen, ob es sich bei dem übertragenen Teilchen um ein Proton oder ein H-Atom handelt. Im Falle eines Protontransfers würde ein polares Lösungsmittel das resultierende Zwitterion stabilisieren, und die langwellige Emission aus dem $\mathrm{S}_{1}^{\prime}$-Zustand verstärkt sein. Man beobachtet jedoch, dass die stationäre Emission im kurzwelligen Bereich der Emissionswellenlängen in Propylencarbonat und Tetrachlorkohlenstoff sehr viel stärker ausgeprägt ist als in Benzylalkohol. Da Benzylalkohol ein kleineres elektrisches Dipolmoment als Propylencarbonat aufweist $\left(\mu_{P}=4.9 \mathrm{D}>\mu_{B}=1.7 \mathrm{D}^{4}[62]\right)$, aber ein größeres als der unpolare Tetrachlorkohlenstoff, kann dieser Effekt nicht mit der Polarität der Lösungsmittel zusammenhängen. Gleiches gilt für den beobachteten Einfluss der Lösungsmittel auf die Fluoreszenzdynamik von 1.8-DHAQ. Grundsätzlich ist dieser Einfluss, wie im Vorangegangenen beschrieben wurde, relativ klein. Die stark ausgeprägte Stokesverschiebung ist bei allen Lösungsmitteln innerhalb der ersten $150 \mathrm{fs}$ weitgehend abgeschlossen. Allerdings zeigt die Entwicklung des Fluoreszenzspektrums von 1.8-DHAQ in Propylencarbonat und Tetrachlorkohlenstoff auch auf der Zeitskala weniger hundert fs noch deutliche Veränderungen. Im Bereich der kurzwelligen Emission ist die Fluoreszenzintensität noch sehr viel stärker als in den jeweiligen stationären Spektren, während das transiente Spektrum in Benzylalkohol bereits eine sehr große Ähnlichkeit mit dem stationären Fall aufweist. Die Umstrukturierung des Molekülgerüstes vollzieht sich demnach in Benzylalkohol schneller als in den anderen beiden Lösungsmitteln. Auch diese Beobachtung kann also nicht mit der Polarität der Lösungsmittel zusammenhängen. Möglich wäre es, dass Benzylalkohol durch die Ausbildung intermolekularer Wasserstoffbrücken mit 1.8-DHAQ, die Potentiallandschaft von Schwingungsmoden, die in den Protontransfer involviert sind beeinflusst. Insgesamt kann aus den beobachteten Lösungsmittelabhängigkeiten also geschlossen werden, dass es sich bei dem aus der Umstrukturierung des angeregten 1.8-DHAQ resultierenden Tautomer mit Bestimmtheit nicht um ein Zwitterion handelt. Nichtsdestotrotz wird sich auch beim Übergang in einen solchen ungeladenen, tautomeren Zustand das Dipolmoment der Probe leicht ändern und zur Stabilisierung des neuen Zustands werden sich die Dipolmomente der umliegenden Lösungsmittelmoleküle entsprechend umorientieren, um die Dipol-DipolWechselwirkung zu minimieren. Unterschiedliche Zeitkonstanten für die Polarisationsrelaxation von Benzylalkohol und Propylencarbonat könnten eine Erklärung

\footnotetext{
${ }^{4} \mathrm{D}=$ debye units, $1 \mathrm{D}=3.33564 \cdot 10^{-30} \mathrm{Cm}$
} 
dafür liefern, dass zur Anpassung der Fluoreszenzkurven im LEW-Bereich in Benzylalkohol vier statt wie in Propylencarbonat drei gefaltete Exponentialkurven nötig sind.

\subsection{Einfluss der Deuterierung}

Nun zu den Folgerungen, die sich aus dem Einfluss der Deuterierung ergeben. Da sich die Deuterierung der Hydroxylgruppen von 1.8-DHAQ merklich auf die stationären spektroskopischen Eigenschaften auswirkt, muss sich der FC-Überlapp zwischen dem angeregten Zustand von 1.8-DHAQ und dem Grundzustand infolgedessen verändert haben. Eine Deuterierung sollte sich auf alle Schwingungszustände auswirken, die eine Bewegung des Protons beinhalten, also neben der $\nu(\mathrm{OH})$-Mode z.B. auch auf die $\delta(\mathrm{C}-\mathrm{O})$-Biegemode des quasiaromatischen Ringes und weitere niederfrequente Gerüstmoden, die eine Bewegung der OH-Gruppen beinhalten. Nach Smulevich et al. lässt sich der Einfluss der Deuterierung auf die stationären Spektren jedoch schon gut im LS-Potentialbild entlang der $\mathrm{r}(\mathrm{OH})$ Koordinate durch eine energetische Absenkung der $\nu(\mathrm{OH})$-Schwingungszustände beschreiben. Die Tatsache, dass andere Moden für die Übergänge aus dem thermischen Gleichgewicht weniger entscheidend sind, sagt jedoch nichts darüber aus ob und inwieweit diese Moden am Erreichen des thermischen Gleichgewichts und an der Umstrukturierung des Moleküls beteiligt sind und durch welche Prozesse die Form des Potentials im elektronisch angeregten Zustand zustandekommt. Aus den stationären Spektren lässt sich daher auch nicht schließen, ob sich ausschließlich das Proton selbst bewegt oder sich eher die Potentiallandschaft um das Proton herum verändert. Hierzu musste die zeitliche Änderung der spektroskopischen Eigenschaften von 1.8-DHAQ untersucht werden. Es zeigte sich, dass die Deuterierung keinen deutlich erkennbaren Einfluß auf die Fluoreszenzdynamik nach $150 \mathrm{fs}$ besitzt. Dies bedeutet, dass der Transfer des Protons bereits innerhalb der ersten 150 fs abgeschlossen, oder aber dass der als solcher bezeichnete Prozess, wie durch Nagaoka et al. vorhergesagt (vgl. Kap. 2.2.3), keine große Verschiebung des Protons beinhaltet, sondern vielmehr eine Deformation des quasiaromatischen Ringes, die andere Freiheitsgrade des Moleküls umfasst als die $\nu(\mathrm{OH})$-Schwingungskoordinate. Eine Entscheidung, welche Erklärung hier zutrifft, kann aufgrund der limitierten Zeitauflösung des Experiments leider nicht getroffen werden. 


\subsection{Intensitätsmodulationen im Pump-Probe- Signal}

Nun zum möglichen Ursprung der im Pump-Pump-Experiment beobachteten Signalmodulation. Solche Signalmodulationen können beobachtet werden, wenn keine stationären Eigenzustände des Systems präpariert werden, sondern mit kurzen Laserpulsen ausreichender spektraler Breite mehrere Niveaus einer Schwingungsmode phasenkohärent angeregt werden und so Schwingungs-Wellenpakete im angeregten Zustand oder im Grundzustand (durch Raman-artige Prozesse) erzeugt werden. Da es sich bei den lokalisierten Wellenpaketen nicht mehr um Eigenzustände des System handelt, entwickeln sie sich abhängig von der Form des Potentials gemäß der zeitabhängigen Schrödingergleichung, und oszillieren innerhalb der Grenzen des Potentials hin und her. Sind die jeweiligen Moden direkt am Pump-Probe-Übergang beteiligt führt dies zu einer Intensitätsmodulation im Pump-Probe-Signal. Bei Wellenpaketen im angeregten Zustand trägt überdies auch noch die periodische Änderung des FC-Überlapp zwischen dem angeregten Zustand und höher angeregten Zuständen mit zur Signalmodulation bei $[63]$.

Es stellt sich nun die Frage, welche Moden von 1.8-DHAQ für die Signalmodulation verantwortlich sind und ob ein Zusammenhang dieser Moden zum Protontransfer besteht. Zur Beschreibung der spektroskopischen Eigenschaften von 1.8-DHAQ hatten Smulevich et al. ein LS-Potential entlang der $\mathrm{r}(\mathrm{OH})$-Koordinate vorgeschlagen. Die $\nu(\mathrm{OH})$-Schwingungsmode mit einer Frequenz von $\simeq 1300 \mathrm{~cm}^{-1} \mathrm{im}$ angeregten Zustand, kann jedoch nicht für die Wellenpakete verantwortlich sein, da die spektrale Breite des anregenden Laserpulses von $\sigma_{F W H M}=400-500 \mathrm{~cm}^{-1}$ kaum für eine kohärente Anregung zweier $\nu(\mathrm{OH})$-Schwingungszustände ausreicht. Überdies würden die Oszillationen in diesem Fall eine Frequenz von $1300 \mathrm{~cm}^{-1}$ besitzen, entsprechend der Energiedifferenz zweier aufeinanderfolgender $\nu(\mathrm{OH})$ Schwingungszustände. Es müssen also andere, niederfrequente Moden für die Schwingungs-Wellenpakete verantwortlich sein. Die stärkste in den Oszillationen des Pump-Probe-Signals beobachtete Frequenz bei $(207-215) \mathrm{cm}^{-1}$ entspricht erstaunlich gut einer von Smulevich et al. bei $(214 \pm 5) \mathrm{cm}^{-1}$ im Grundzustand von 1.8-DHAQ gefundenen Mode. Da bei Probenwellenlängen weit außerhalb des stationären Absorptionsspektrums beobachtete wurde, kann für die Oszillationen keine Wellenpaketdynamik im Grundzustand verantwortlich sein. Im angeregten Zustand hingegen sollte die entsprechende Mode nach Smulevich et al. eine Frequenz von $242 \mathrm{~cm}^{-1}$ besitzen. Eine Erklärung hierfür wäre, dass das Potential stark anharmonisch ist, und die Energiedifferenz $\Delta \mathrm{E}$ zwischen benachbarten $\mathrm{Ni}$ veaus mit zunehmender Schwingungsquantenzahl kleiner wird.

Diese bei $(214 \pm 5) \mathrm{cm}^{-1}$ im Grundzustand von 1.8-DHAQ gefundene Mode wur- 
de von Smulevich et al. in früheren Veröffentlichungen einer Biegeschwingung in der Ebene des Molekülgerüstes im elektronischen Grundzustand zugeordnet, die große Bewegungen der beiden O-H-Gruppen beinhaltet. In der letzten Veröffentlichung wurde hingegen für dieselbe Bande die $\delta(C-O)$-Biegeschwingung verantwortlich gemacht. Beiden Zuordnungen ist also gemeinsam, dass durch ein Schwingungswellenpaket dieser Mode der Abstand der Sauerstoffatome im durch Wasserstoffbrücken gebundenen $\mathrm{C}-\mathrm{O}-\mathrm{H} \cdots \mathrm{O}=\mathrm{C}-\mathrm{Ring}$ moduliert wird.

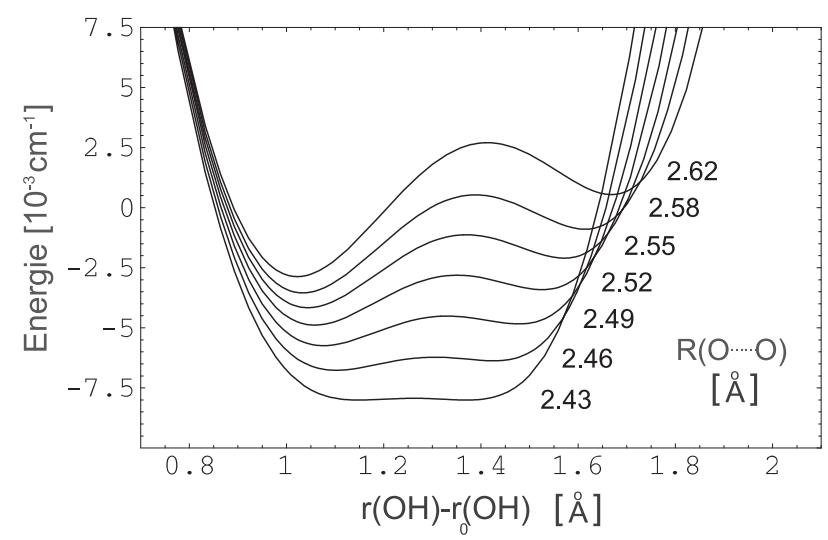

Abbildung 5.2: Abhängigkeit des LS-Potentials vom R(O..O)-Abstand. Mit Verringerung des $R(O \cdots O)$-Abstands verschwindet die Barriere (vgl. Anhang B).

Im folgenden soll erörtert werden, auf welche Weise die mutmaßliche $\delta(C-O)$ Mode zu den beobachteten Signalmodulationen führen kann. In den Interpretationsansätzen von Smulevich et al. wurde der Protontransfer als ein eindimensionales Problem behandelt, in dem der Verlauf des Potentials entlang der $\mathrm{r}(\mathrm{OH})$ Koordinate betrachtet wurde, und ein fester $\mathrm{R}(\mathrm{O} \cdots \mathrm{O})$-Abstand der Sauerstoffatome im wasserstoffbrückengebundenen $\mathrm{C}_{1 / 8}-\mathrm{O}-\mathrm{H} \cdots \mathrm{O}=\mathrm{C}_{9}$ Ring im angeregten Zustand angenommen wurde. Da die Höhe und Breite der Potentialbarriere

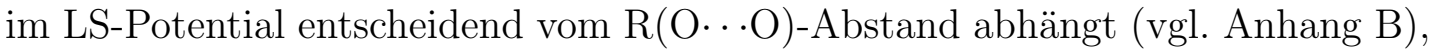
denn von diesem hängt die Stärke der Wasserstoffbrückenbindung ab, wird eine kohärente Anregung von Schwingungsfreiheitsgraden, die den $\mathrm{R}(\mathrm{O} \cdots \mathrm{O})$-Abstand modulieren, zu einer Modulation des Potentialverlaufs entlang der $\mathrm{r}(\mathrm{OH})$-Koordinate führen (vgl. Abb. 5.2). Diese wird eine periodischen Änderung der Frequenz der $\nu(\mathrm{OH})$-Schwingungsmode bewirken, was wiederum eine periodischen Änderung der Übergangsfrequenz in den Grundzustand (bzw. höhere elektronische Zustände) zur Folge hätte. Letztlich wird genau diese Änderung der Übergangsfrequenz als Intensitätsmodulation im Pump-Probe-Signal registriert, da bei einer festen Wellenlänge beobachtet wird. Solche Intensitätsmodulationen im Pump-Probe-Signal aufgrund einer starken anharmonischen Kopplung zwischen der hochfrequenten $\nu(\mathrm{OH})$-Schwingungsmode und anderen niederfrequenten Moden, die den Abstand der Wasserstoffbrückenbindung modulieren, wurden bereits 
bei anderer Molekülen mit intramolekularen Wasserstoffbrückenbindungen gefunden [64-66]. Es existieren Modelle für den ESIPT, nach denen die Entwicklung eines angeregten Wellenpakets entlang der Auslenkungskoordinate einer niederfrequenten Mode zu einer Änderung der Potentialfläche entlang der gekoppelten Schwingungskoordinate höherer Frequenz führt und hierdurch erst ein barriereloser Kanal für den Protontransfer geöffnet wird [11,26,67,68]. Die maximale, für den Protontransfer benötigte Zeit entspricht dann der halben Schwingungsdauer der niederfrequenten Mode zuzüglich der Zeit, die das Wellenpaket benötigt, um sich entlang des barrierelosen Kanals zum Potentialminimum des Tautomers zu bewegen.

Wendet man dieses Modell auf die Prozesse in 1.8-DHAQ an, so folgt dass erst die Verringerung des $\mathrm{R}(\mathrm{O} \cdots \mathrm{O})$-Abstandes durch entsprechend angeregte Moden die Barriere zwischen $S_{1}$ und $S_{1}^{\prime}$ für die untersten vibronischen Zustände zum Verschwinden bringen würde. Nach Smulevich und Gillespie sollte eine solche Barriere, sofern sie bestünde, sehr klein sein $\left(<475 \mathrm{~cm}^{-1}\right)$ und die den $\mathrm{R}(\mathrm{O} \cdots \mathrm{O})$ Abstand modulierende $214 \mathrm{~cm}^{-1}$-Mode würde eine solche Barriere quasi schon nach 80 fs das erste mal zum verschwinden bringen, d.h. die Wellenfunktion hätte sich innerhalb etwa $(80+13)$ fs delokalisiert. Da jedoch in diesem Experiment mit einem hohen Überschuss an Schwingungsenergie angeregt wurde (s.o.), sollte sich der präparierte Zustand bereits vor der Modulation des $\mathrm{R}(\mathrm{O} \cdots \mathrm{O})$-Abstandes oberhalb der Barriere befinden, und der Protontransfer eher einer barrierelosen Relaxation entsprechen. An dieser Stelle wird deutlich, dass es sehr interessant wäre, die Experimente nochmals mit niedrigeren Anregungsenergien zu wiederholen.

Doch nun zur Frage nach dem Auslöser der niederfrequenten Wellenpakete, die im Vorherigen als Verursacher der beobachteten Signalmodulationen angeführt wurden. Eine niederfrequente Mode von $(214 \pm 5) \mathrm{cm}^{-1}$ könnte innerhalb der spektralen Breite des anregenden Laserpulses von 400-500 $\mathrm{cm}^{-1}$ (FWHM) direkt angeregt werden, sofern sie einen endlichen FC-Überlapp mit dem elektronisch Grundzustand aufweist. Allerdings wurde in den stationären Anregungsspektren von Smulevich et al. und Gillespie et al. kein Hinweis auf ihre Existenz gefunden, was auf einen verschwindend kleinen FC-Überlapp mit dem Grundzustand schließen läßt. Die Anregung niederfrequenten Moden durch sehr schnelle IVR-Prozessen infolge der starken anharmonischen Kopplung mit der direkt angeregten $\nu(\mathrm{OH})$ Schwingungsmode scheint aber auch fragwürdig, da eine solche Anregung eher inkohärent erfolgen sollte. Es wurde auch vorgeschlagen, dass niederfrequente Gerüstmoden das Resultat des Protontransfers sind, da im Verlauf der strukturellen Umorganisation des Moleküls eine Verschiebung des Potentialminimums bestimmter niederfrequenter Gerüstmoden eintritt und das Molekül daher beginnt um die neuen Minima zu oszillieren $[19,26]$. Eine Verschiebung des Potentialminimums bestimmter niederfrequenter Gerüstmoden könnte aber auch schon durch 
die instantan nach der Anregung stattfindende Delokalisierung der $\pi \pi^{*}$ Elektronen wie sie von Nagashima et al. vorhergesagt wurde ausgelöst werden.

Eine Möglichkeit dem Auslöser der Oszillation auf die Spur zu kommen, könnte darin bestehen die Phase der Oszillation zu bestimmen, denn zum Zeitpunkt der Präparation des Wellenpaketes sollte sich die Schwingung an einem Umkehrpunkt befinden. Leider kann aus den in Kapitel 4.1.3 geschilderten Gründen die Phase der Oszillation in dem hier durchgeführten Pump-Probe-Experiment nicht genau bestimmt werden, daher liegen beide Varianten, die der niederfrequenten Schwingung als Wegbereiter des Protontransfers und die der niederfrequenten Schwingung als Konsequenz des Protontransfers im Rahmen des Möglichen.

Aus der vorangegangenen Diskussion ergibt sich, dass der Begriff des Protontransfers einer genaueren Definition der mit ihm einhergehenden Umstrukturierungen bedarf. In der klassischen Beschreibung bewegt sich ein Proton oder ein neutrales H-Atom entlang der Wasserstoffbrücken-gebundenen O-H...O als Reaktionskoordinate. Nach dem Transfer sind die Rollen der vorherigen kovalenten Bindung und Wasserstoffbrückenbindung vertauscht. Hierbei stellt sich natürlich die Frage, ob die Elektronenverteilung der Protonbewegung adiabatisch folgt, oder ob infolge der Anregung eine Umstrukturierung der Elektronendichteverteilung eintritt, die die Verschiebung der Protonposition erst nach sich zieht. Nach neueren Modellen sind auch Kernbewegungen des gesamten Molekülgerüstes in die Umstrukturierung involviert und es findet eine globale Umverteilung der Elektronendichten im gesamten Molekül mit nachfolgender Relaxation entlang verschiedener gekoppelter Schwingungskoordinaten statt. Wenn man von einer für den "Protontransfer" notwendigen Zeit sprechen will, muss man sich zuvor erst darauf einigen, welche Vorgänge man diesem zuordnet und wann man ihn als abgeschlossen betrachtet. Diesen Zeitpunkt kann man entweder als das Ende der Verschiebung der Elektronendichte definieren oder durch das Erreichen des Potentialminimums entlang der $\mathrm{r}(\mathrm{OH})$-Koordinate, wobei sich letzteres aufgrund der $\mathrm{R}(\mathrm{O} \cdots \mathrm{O})$-Modulation noch bis zum Abklingen der verantwortlichen Moden ändert. In einer weitergehenden Definition würde man das Erreichen des globalen Minimums entlang der multidimensionalen Potentiallandschaft annehmen. Letztlich gehen die Verschiebung des Protons und die Schwingungsrelaxation des Tautomers entlang verschiedener Schwingungskoordinaten nahtlos ineinander über. 


\section{Kapitel 6}

\section{Zusammenfassung und Ausblick}

In der vorliegenden Arbeit wurde das strahlungslose Relaxationsverhalten in Zusammenhang mit dem intramolekularen Protontransfer im ersten elektronisch angeregten Zustand von 1.8-Dihydroxy-9.10-Anthrachinon untersucht. Hierzu wurde das zeitabhängige Transmissionsverhalten von 1.8-DHAQ in Benzylalkohol (Pump-Probe-Messungen) sowie die zeitaufgelöste Fluoreszenzdynamik in den drei verschiedenen Lösungsmitteln Benzylalkohol, Propylencarbonat und Tetrachlorkohlenstoff (Fluoreszenz-Aufkonvertierungs-Messungen) analysiert. Überdies wurde der Einfluss der Deuterierung der Hydroxylgruppen von 1.8-DHAQ in Tetrachlorkohlenstoff betrachtet.

Aus den experimentellen Beobachtungen ergibt sich das folgende Bild für den Protontransfer im angeregten Zustand von 1.8-DHAQ. Bereits innerhalb der ersten 150 fs nach der Anregung finden wesentliche Umstrukturierungen des 1.8DHAQ-Moleküls statt, die eine Delokalisierung der Aufenthaltswahrscheinlichkeit des Protons zwischen den beiden Sauerstoffatomen des quasiaromatischen Ringes zur Folge haben. Ursache hierfür ist höchstwahrscheinlich eine in theoretischen Modellen postulierte Delokalisierung der $\pi \pi^{*}$-Elektronen. Letztere liefert auch eine zufriedenstellende Erklärung für die Beobachtung von Wellenpaketen in nicht Franck-Condon-aktiven, niederfrequenten Schwingungsmoden. Auf einer Zeitskala von 200-400 fs nach der Anregung findet durch nichtresonante IVR verursacht eine weitere Verschiebung des Proton in Richtung des ehemaligen Carbonyl-Sauerstoffatoms statt, an der mehrere niederfrequente Schwingungsmoden des Moleküls beteiligt sind. Die Frage, ob am Protontransfer hauptsächlich Schwingungsmoden des wasserstoffbrückengebundenen, quasiaromatischen Ringes beteiligt sind oder des gesamten Molekülgerüstes, kann hier, da die theoretische Zuordnung der beobachteten Schwingungsmoden nicht eindeutig ist, leider nicht beantwortet werden. Sicher ist aber, dass an der Umstrukturierung des Moleküls und dem Erreichen des Tautomer-Minimums neben der $\nu(\mathrm{OH})$-Mode auch weitere Schwingungsmoden von 1.8-DHAQ beteiligt sind und sich das Molekül entlang einer multidimensionalen Potentialfläche auf das Tautomer-Minimum im 
angeregten Zustand zubewegt. Die Verteilung der Energie auf mehrere Freiheitsgrade des Moleküls ist auch der Grund dafür, weshalb ein Rücktransfer des Protons sehr unwahrscheinlich wird und innerhalb der Lebensdauer des angeregten Zustands nicht beobachtet werden kann. Die Lösungsmittelabhängigkeit der Dynamik legt nahe, dass es sich bei dem aus der Umstrukturierung des angeregten 1.8-DHAQ resultierenden Tautomer mit Bestimmtheit nicht um ein Zwitterion handelt. Dies ist konsistent mit der Annahme eines zwischen beiden Sauerstoffatomen des quasiaromatischen Ringes delokalisierten Protons und zweier über das gesamte aromatische Ringsystem delokalisierter $\pi \pi^{*}$-Elektronen. Die hier gefundenen geringen Deuterierungseffekte unterstützen ebenfalls diese Interpretation.

Zusammenfassend ist zu sagen, dass sich der Protontransfer im angeregten $\mathrm{Zu}$ stand von 1.8-DHAQ weder, wie aus den stationären Messungen gefolgert wurde, durch eine separate Verschiebung des Protons innerhalb des quasiaromatischen Ringes in seiner Gesamtheit beschreiben lässt, noch, wie in der Theorie vermutet wurde, durch eine reine Deformation des Molekülgerüstes. Vielmehr umfasst die Umstrukturierung im angeregten Zustand mehrere Schwingungsfreiheitsgrade des Moleküls, die $\nu(\mathrm{OH})$-Mode ebenso wie andere niederfrequente Schwingungsmoden, und kann nur auf der Grundlage einer multidimensionalen Potentialfläche vollständig erklärt werden.

Abschließend lässt sich Folgendes anmerken: Da in den durchgeführten Experimenten mit einem hohen Überschuss an Schwingungsenergie angeregt wurde, tritt der als Protontransfer bezeichnete Prozess hier als barrierelose Relaxation in Erscheinung. Es kann jedoch keine Aussage darüber gemacht werden, ob sich unterhalb einer gewissen Schwellenenergie nicht doch noch eine kleine Barriere zeigt. Es wäre daher sehr aufschlussreich weitere zeitaufgelöste Messungen bei kleineren Anregungsenergien durchzuführen. In diesem Zusammenhang könnte auch festgestellt werden, ob die beobachteten Oszillationen erst oberhalb einer bestimmten Anregungsenergie einsetzen, da hieraus weitere Rückschlüsse über ihren Ursprung gezogen werden könnten. Voraussetzung für ein tieferes Verständnis des hier untersuchten Protontransferprozesses wäre jedoch eine Annäherung an das Molekül auf theoretischer Ebene. Ab initio Rechnungen für den angeregten Zustand des Moleküls könnten hierzu einen wesentlichen Beitrag leisten. 
Anhang A

\section{Tabellen}




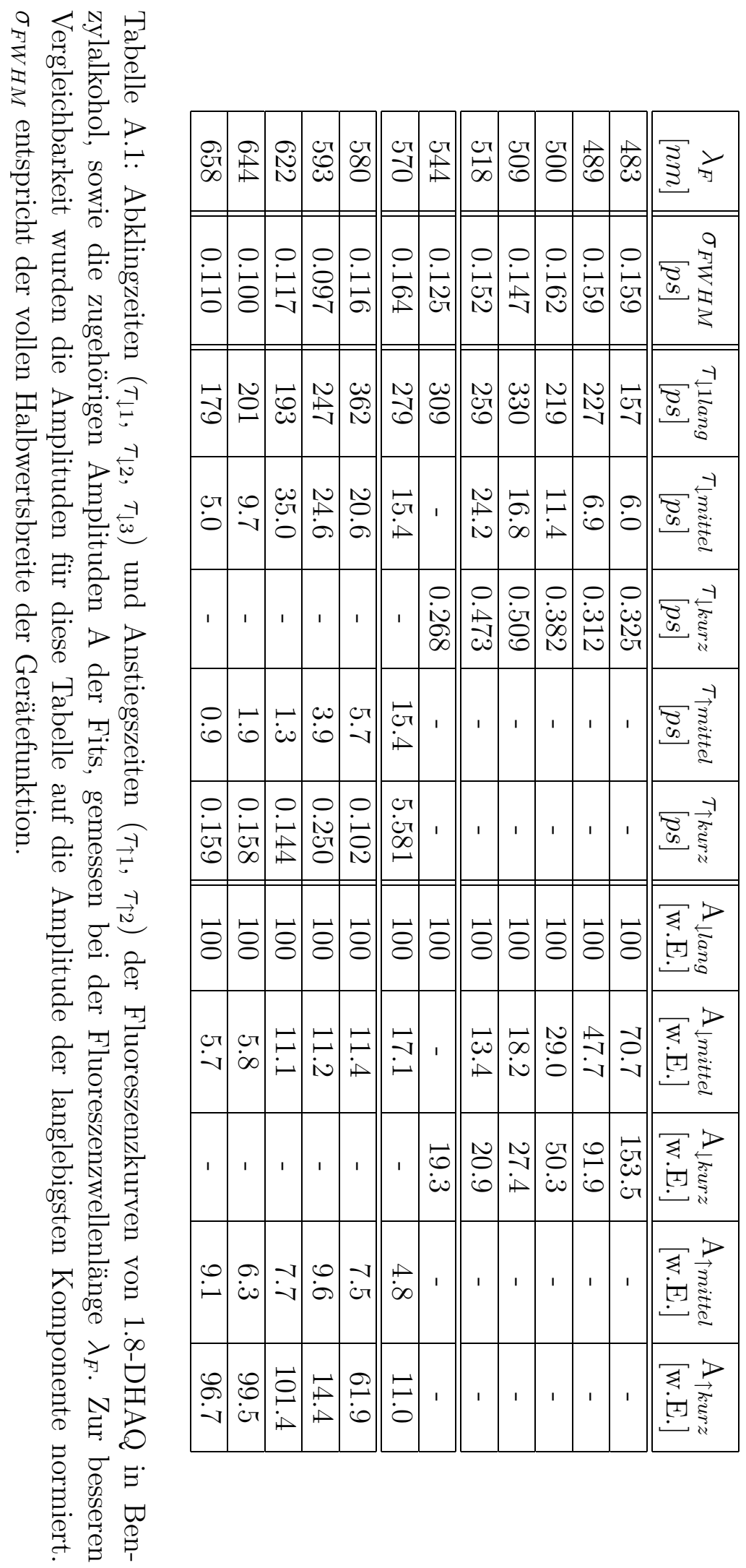




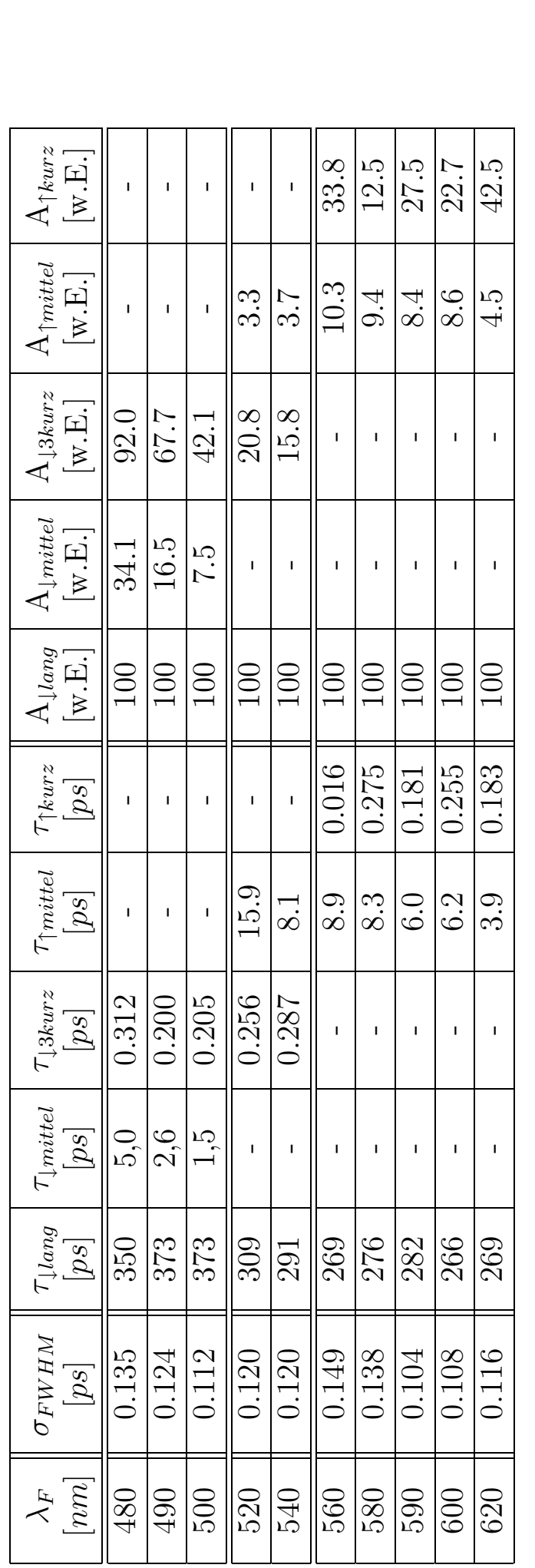

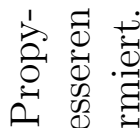

그 일 零 $\infty$ o 0 : 元 를 व 弪

证 ¿ 过 压它莺 ช્屯 (동 छ E क क

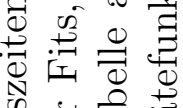
के प्र 岂艺兽

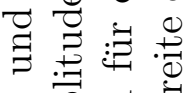
郘

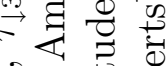
$\stackrel{\mathrm{N}}{\rightarrow}$

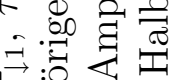
궁 $\frac{8}{3}=\frac{0}{0}$ ․ㅠㅇ

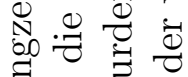

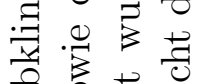

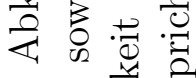
ن 율

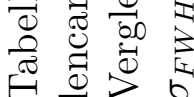




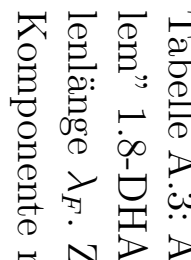

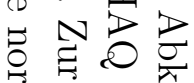

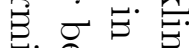

․․요

+ 胥通.

वे 80

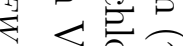

बैद

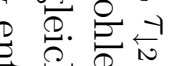

क 8

‥ 웡

题

\&

¿

응 号 号.

을 능

I1 0 o

节苛:

总要

$\overrightarrow{+} \vec{\Xi}$

है

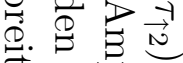

止兽通

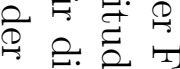

(2)

娄 $\rightarrow$

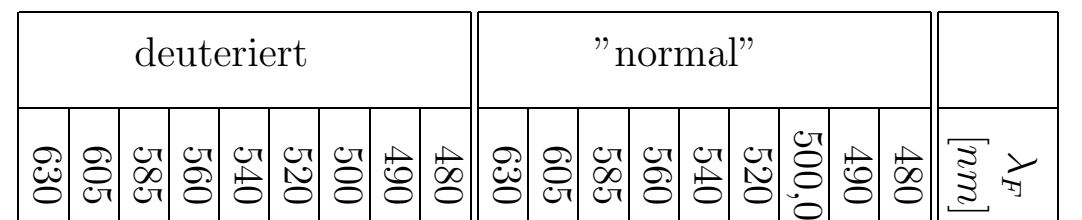

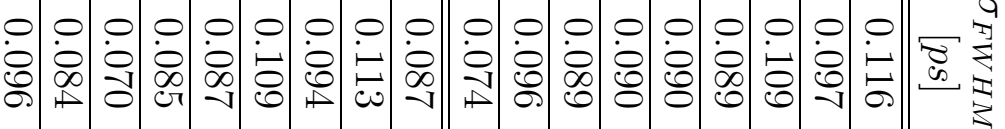

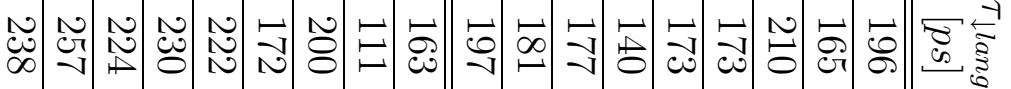

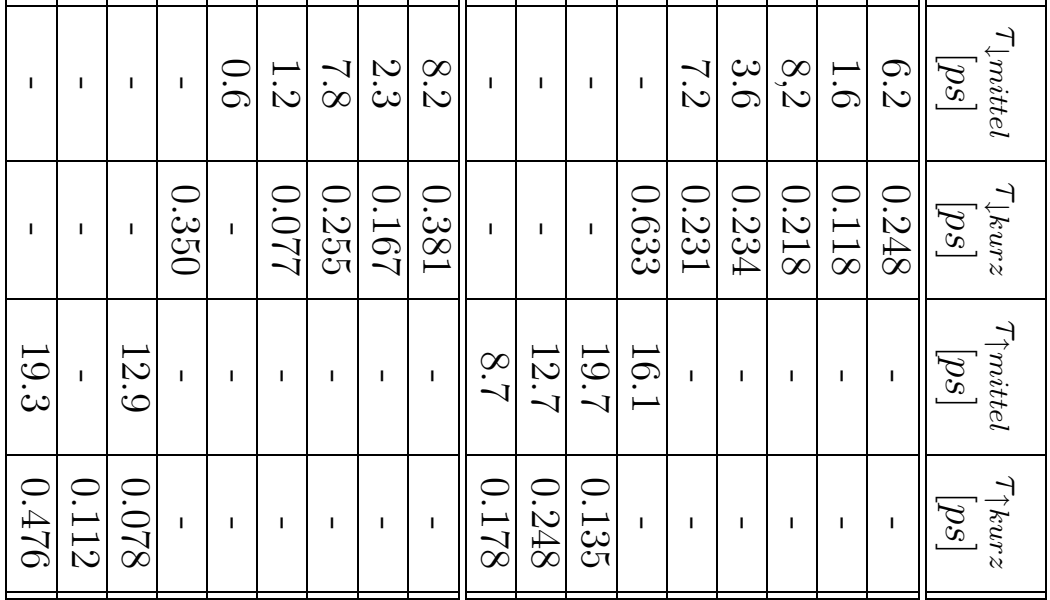

ஏे

?

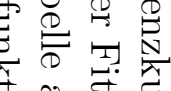

स.

क्ष

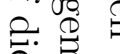

$>$ 贾

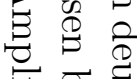

.

के จ

की

ڤ艹

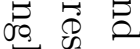

क

옹. 蛋

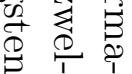

\begin{tabular}{|c|c|c|c|c|c|c|c|c|c|c|c|c|c|c|c|c|}
\hline ' & 1 & 1 & I & $\stackrel{\sim}{\stackrel{\sim}{c}}$ & $\begin{array}{l}N \\
N \\
\sim\end{array}$ & $\begin{array}{l}\stackrel{N}{\bullet} \\
\bullet\end{array}$ & & لـ & 1 & 1 & I & $\stackrel{\oplus}{\dot{c} 0}$ & $\begin{array}{l}N \\
0\end{array}$ & $\begin{array}{ll}\vec{B} \\
\dot{\sigma}\end{array}$ & & 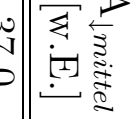 \\
\hline 1 & 1 & ' & $\vec{\sigma}$ & 1 & $\begin{array}{l}0 \\
\tilde{a} \\
0\end{array}$ & $\underset{c}{\stackrel{T}{c}}$ & $\begin{array}{l}\overrightarrow{\vec{\sigma}} \\
\dot{0}\end{array}$ & $\begin{array}{l}0 \\
c \\
i \\
i\end{array}$ & 1 & 1 & $\hat{c}$ & $\begin{array}{ll}0 \\
0 \\
0 & 0 \\
0\end{array}$ & $\left|\begin{array}{c|}1 \\
c \\
\infty \\
\infty\end{array}\right|$ & $\begin{array}{l}\stackrel{t}{*} \\
\stackrel{i}{*}\end{array}$ & 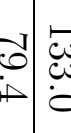 & 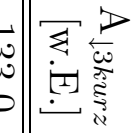 \\
\hline$\stackrel{0}{0}$ & & $\begin{array}{l}\vec{N} \\
\stackrel{v}{v}\end{array}$ & ' & ' & 1 & 1 & 1 & & 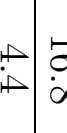 & $\begin{array}{c}5 \\
\infty\end{array}$ & $\begin{array}{ll}\vec{P} \\
\vec{P}\end{array}$ & $\vec{~}$ & ' & 1 & 1 & 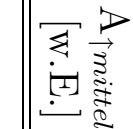 \\
\hline טָ & $\begin{array}{l}\stackrel{N}{\infty} \\
\bullet \\
\bullet\end{array}$ & $\begin{array}{l}\vec{v} \\
\vec{v} \\
0\end{array}$ & ' & ' & 1 & 1 & 1 & ' & 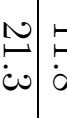 & $=$ & $\begin{array}{l}\vec{b} \\
0\end{array}$ & 1 & ' & 1 & ' & 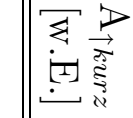 \\
\hline
\end{tabular}




\section{Anhang B}

\section{Lippincott-Schröder-Potential}

Mitte der 60er Jahre schlugen Lippincott und Schroeder ein eindimensionales Modell zur Beschreibung von Wasserstoffbrückenbindungen vor, das sie auf lineare und gewinkelte Wasserstoffbrückenbindungssysteme der Art X-H..Y anwendeten $[42,43]$.

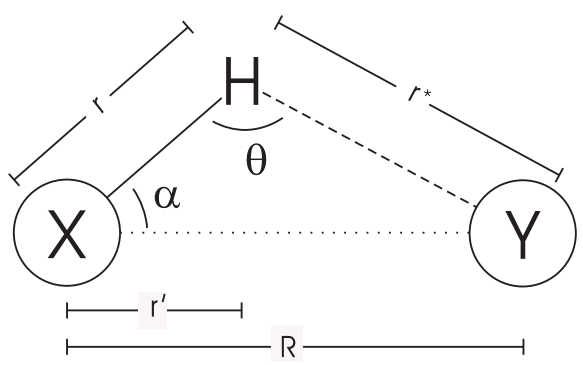

Abbildung B.1: Koordinaten des Wasserstoffbrückenbindungssystems $X-H \cdots Y$.

Sie nahmen hierbei an, dass sich die Änderung der potentielle Energie infolge einer Dehnung der Bindung, sowohl für die kovalente Bindung zwischen $\mathrm{X}$ und $\mathrm{H}$, als auch für die schächere Wasserstoffbrückenbindung zwischen $\mathrm{H}$ und $\mathrm{Y}$, durch ein Potential der Form

$$
V\left(r^{\prime}\right)=D\left[1-\exp \left(-n \Delta r^{2} / 2 r^{\prime}\right)\right]
$$

beschreiben lässt. D entspricht hierbei der Dissoziationsenergie der Bindung, $\Delta r=r^{\prime}-r_{0}$ der Auslenkung relativ zum Gleichgewichtsabstand $r_{0}$ der gebundenen Atome, und $\mathrm{n}=k_{0} r_{0} / D$ enthält die Kraftkonstante $k_{0}$ der freien Schwingung entlang der Bindung. Die Van-der-Waals-Abstoßung zwischen den Atomen X und Y beschrieben Lippincott und Schroeder durch ein exponentielles Potential der Form $\mathrm{V}_{X Y, r e p}(\mathrm{R}) \sim \exp (-\mathrm{b} / \mathrm{R})$, mit $\mathrm{b}$ als repulsiven Parameter zwischen $\mathrm{X}$ und $\mathrm{Y}$, und dem Abstand $\mathrm{R}$ der Atome $\mathrm{X}$ und $\mathrm{Y}$. Ein attraktives elektrostatisches 
Potential zwischen $\mathrm{X}$ und $\mathrm{Y}$ in der resonanten Form $\mathrm{O}^{-} \cdots \mathrm{H}_{-} \mathrm{O}^{+}$berücksichtigten sie durch einen Term $\mathrm{V}_{X Y, a t t r}(\mathrm{R}) \sim(1 / \mathrm{R})$. Die Gesamtenergie $\mathrm{V}_{X H Y}\left(\mathrm{r}^{\prime}, \mathrm{R}\right)$ des dreiatomigen Systems entlang r' (H-Auslenkung in Richtung von Y) ergibt sich dann als

$$
V_{X-H \ldots Y}\left(r^{\prime}, R\right)=V_{X H}\left(r^{\prime}\right)+V_{H Y}\left(r^{\prime}, R\right)+V_{X Y, r e p}\left(r^{\prime}, R\right)+V_{X Y, a t t r}\left(r^{\prime}, R\right)
$$

Der Potentialverlauf entlang r' ist der eines Doppelminimumpotentials. Die beiden Minima entsprechen den beiden resonanten Formen $\mathrm{O}-\mathrm{H} \cdots \mathrm{O}$ und $\mathrm{O}^{-} \cdot \cdots \mathrm{H}_{-} \mathrm{O}^{+}$. In gewinkelten Systemen, wie dem in Abbildung B.1 dargestellten, schreibt sich der Beitrag des freien X-H-Potential als

$$
V_{X H}\left(r^{\prime}\right)=D\left[1-\exp \left(-n\left(r^{\prime}-r_{0} \cos \alpha\right)^{2} / 2 r^{\prime} \cos \alpha\right)\right]
$$

und der Beitrag des H...Y-Potential als

$$
V_{H Y}\left(r^{\prime}, R\right)=D^{*}\left[1-\exp \left(-n^{*}\left(R-r^{\prime}-r_{0}^{*} \cos (\alpha+\theta)\right)^{2} / 2\left(R-r^{\prime}\right) \cos (\alpha+\theta)\right)\right]
$$

Hier entsprechen $\mathrm{D}^{*}, \mathrm{n}^{*}, \mathrm{r}^{*}$ den zu D, n, r korrespondierenden Größen für die H-O $\mathrm{O}^{+}$ Bindung. Das Lippincott-Schröder-Potential (LS-Potential) diente Smulevich et al. als Grundlage zur Beschreibung der stationären Spektren von 1.8-DHAQ (vgl. Kap. 2.2.3). 


\section{Literaturverzeichnis}

[1] R.P. Bell. The proton in chemistry. Methuen \& Co. Ltd.: London, 1959.

[2] E.F. Caldin and V. Gold. Protontransfer reactions. Chapman \& Hall: London, 1975.

[3] R. Stewart. The proton: applications to organic chemistry. Academic Press: Orlando, 1985.

[4] T. Bountis. Proton-transfer in hydrogen-bonded systems. NATO ASI Series (B291), Plenum Press: New York and London, 1991.

[5] T.P. Smith, K.A. Zaklika, K. Thakur, G.C. Walker, K. Tominaga, and P.F. Barbara. Ultrafast studies on proton transfer in photostabilizers. J.Photochem.Photobiol.A, 65:165ff., 1992.

[6] P. Mitchell. Coupling of phosphorylation to electron and hydrogen transfer by a chemiosmotic type of mechanism. Nature: London, 191:144-148, 1961.

[7] A. Weller. Intramolekularer Protonenübergang im angeregten Zustand. Zeitschrift für Elektrochemie, 60:1144-1147, 1956.

[8] P.F. Barbara, P.K. Walsh, and L.E. Brus. Picosecond kinetic and vibrationally resolved spectroscopic studies of intramolecular excited-state hydrogen atom transfer. J.Phys.Chem., 93:29-34, 1989.

[9] S.J. Formosinho and L.G. Arnaut. Excited-state proton transfer reactions II : intramolecular reactions. J.Photochem.Photobiol.A, 75:21-48, 1993.

[10] D. LeGourrierec, S.M. Ormson, and R.G. Brown. Excited state intramolecular proton transfer part 2 : ESIPT to oxygen. Prog.Reaktion Kinetics, 19:211-275, 1994.

[11] A. Douhal, F. Lahmani, and A.H. Zewail. Proton-transfer reaction dynamics. Chem.Phys., 207:477-498, 1996.

[12] H.H. Limbach and J. Manz. Hydrogen transfer: experiment and theory. Ber.Bunsenges.Phys.Chem., 102:289-291, 1998. 
[13] T. Inabe, T. Sekikawa, and T. Kobayashi. Femtosecond fluorescence study of the substitution effect on the proton transfer in thermochromic salicylideneaniline crystals. J.Phys.Chem.A, 101:644-649, 1997.

[14] S. Takeuchi and T. Tahara. Femtosecond ultraviolet-visible fluorescence study of excited-state proton-transfer reaction of 7-azaindole dimer. J.Phys.Chem.A, 102:7740-7753, 1998.

[15] A.V. Smirnov, K. Das, D.S. English, Z. Wan, G.A. Kraus, and J.W. Petrich. Excited-state intramolecular H-atom transfer of Hypericin and Hypocrellin A investigated by fluorescence upconversion. J.Phys.Chem.A, 103:7949-7957, 1999.

[16] T. Fiebig, M. Chachisvilis, M. Manger, and A.H. Zewail. Femtosecond dynamics of double proton transfer in a model DNA base pair: 7-azaindole dimers in the condensed phase. J.Phys.Chem., 103:7419-7431, 1999.

[17] A.L. Sobolewski and W. Domcke. Ab initio potential-energy functions for excited state intramolecular proton transfer: a comparative study of o-hydroxybenzaldehyde, salicylic acid and 7-hydroxy-1-indanone. Phys.Chem.Chem.Phys., 13:3065-3072, 1999.

[18] M. Pfeiffer, C. Chudoba, A. Lau, and T. Elsaesser. Coherent motion of low frequency vibrations in ultrafast excited state proton transfer. Laser Chem., pages 101-103, 1999.

[19] S. Lochbrunner, A.J. Wurzer, and E. Riedle. Ultrafast excitedstate proton transfer and subsequent coherent skeletal motion of 2-(2'hydroxyphenyl)benzothiazole. J.Chem.Phys., 112:10699-10702, 2000.

[20] D. Marks, H. Zhang, P. Borowicz, J. Waluk, and M. Glasbeek. (Sub)picosecond fluorescence upconversion studies of dipyrido[2,3-a:3',2'i]carbazole and related compounds. J.Phys.Chem.A, 104:7167-7175, 2000.

[21] F.V.R. Neuwahl, L. Bussotti, R. Righini, and G. Buntinx. Ultrafast proton transfer in the $\mathrm{s}_{1}$ state of 1-chloroacetylaminoanthraquinone. Phys.Chem.Chem.Phys., 3:1277-1283, 2001.

[22] S. Lochbrunner, T. Schulz, M. Schmitt, J.P. Shaffer, M.Z. Zgierski, and A. Stolow. Dynamics of excited-state proton transfer systems via timeresolved photoelectron spectroscopy. J.Chem.Phys., 114:2519-2522, 2001.

[23] N.P. Ernsting, S.A. Kovalenko, T. Senyushkina, J. Saam, and V. Farztdinov. Wave-packet-assisted decomposition of femtosecond transient ultravioletvisible absorption spectra: application to excited-state intramolecular proton transfer in solution. J.Phys.Chem.A, 105:3443-3453, 2001. 
[24] S. Ameer-Beg, S.M. Ormson, R.G. Brown, P. Matousek, M. Towrie, E.T.J. Nibbering, P. Foggi, and F.V.R. Neuwahl. Ultrafast measurements of excited state intramolecular proton transfer (ESIPT) in room temperature solutions of 3-hydroxyflavone and derivatives. J.Phys.Chem.A, 105:3709-3718, 2001.

[25] P.T. Chou, Y.C. Chen, W.S. Yu, Y.H. Chou, C.Y. Wei, and Y.M. Cheng. Excited-state intramolecular proton transfer in 10-hydrobenzo[h]quinoline. J.Phys.Chem.A, 105:1731-1740, 2001.

[26] S. Lochbrunner, K. Stock, V. de Waele, and E. Riedle. Ultrafast excited state proton transfer: reactive dynamics by multidimensional wavepacket motion. In A. Douhal and J. Santamaria, editors, Femtochemistry and Femtobiology, pages 202-212. World Scientific Publishing, 2002.

[27] A.B. Myers. Molecular electronic spectral broadening in liquids and glasses. Ann.Rev.Phys.Chem., 49:267-295, 1998.

[28] A.H. Zewail. Laser femtochemistry. Science,New Series, 242:1645-1653, 1988.

[29] A.H. Zewail. Femtochemistry, chapter Femtochemistry: atomic-scale dynamics of the chemical bond using ultrafast lasers (nobel lecture), pages 1-83. Wiley-VCH, 2001.

[30] H. Mahr and M.D. Hirsch. An optical upconversion light gate with picosecond resolution. Optics Commun., 13:96-99, 1975.

[31] M.P. Marzocchi and G. Smulevich. Single crystal and polarized infrared spectra of two forms of 1,8-dihydroxyanthraquinone. Vibrational assignment and crystal structures. Chem.Phys., 94:99-108, 1985.

[32] G. Smulevich. Resonance raman and fluorescence spectra of 1.8dihydroxyanthraquinone in n-alkanes at low temperature. Evidence for excited state proton transfer. J.Chem.Phys., 82:14-21, 1985.

[33] G. Smulevich and M.P. Marzocchi. Raman excitation profiles of 1,8dihydroxyanthraquinones at 8k. Chem.Phys., 105:159-171, 1986.

[34] P. Foggi, A. Feis, M.P. Marzocchi, and G. Smulevich. Fluorescence excitation and emission spectra of 1,8-dihydroxyanthraquinone-d0 and -d2 in n-octane at 10 k. J.Chem.Phys., 87:5664-5669, 1987.

[35] M.P. Marzocchi, A.R. Mantini, M. Casu, and G. Smulevich. Intramolecular hydrogen bonding and excited state proton transfer in hydroxyanthraquinones as studied by electronic spectra, resonance raman scattering and transform analysis. J.Chem.Phys., 108:534-549, 1998. 
[36] F. Madsen, I. Terpager, K. Olskaer, and J. Spanget-Larsen. Ultravioletvisible and infrared linear dichroism spectroscopy of 1,8-dihydroxy-9,10anthtraquinone aligned in stretched polyethylene. Chem.Phys., 165:351-360, 1992.

[37] P.A.B. Marasinghe and G.D. Gillispie. Intramolecular hydrogen bonding. IX. Theoretical geometries of substituted anthraquinones relevant to proton transfer studies. Chem.Phys., 136:249-257, 1989.

[38] G.D. Gillispie, N. Balakrishnan, and M. Vangsness. Intramolecular hydrogen bonding. X. Comparison of free jet and shpol'skii matrix electronic spectra of 1,8-dihydroxyanthraquinone. Chem.Phys., 136:259-269, 1989.

[39] J.F. Johnson and A.C. Griffin, editors. Liquid crystals and ordered fluids, volume 4. Plenum Press: New York, 1985.

[40] J.W. Lown, editor. Anthracycline and anthracenedione-based anticancer agents. Elsevier: Amsterdam, 1988.

[41] M.L. Ferreiro and J. Rodriguez-Otero. Ab initio studie of the intramolecular proton transfer in dihydroxyanthraquinones. J.of Molec.Struct., 542:63-77, 2001.

[42] E.R. Lippincott and R. Schroeder. One-dimensional model of the hydrogen bond. J.Chem.Phys., 23:1099-1106, 1955.

[43] E.R. Lippincott and R. Schroeder. Potential function model of hydrogen bonds. J.Phys.Chem., 61:921-928, 1957.

[44] T. Saitoh, K. Mori, and R. Itoh. Two dimensional vibrational analysis of the Lippincott-Schröder potential for OH-O, NH-O and NH-N hydrogen bonds and the deuterium isotope effect. Chem. Phys., 60:161-180, 1981.

[45] D.K. Palit, H. Pal, T. Mukherjee, and J.P. Mittal. Photodynamics of the s1 state of some hydroxy- and amino-substituted naphthoquinones and anthraquinones. J.Chem.Soc.Faraday Trans., 86:3861-3869, 1990.

[46] J. Jethwa, D. Ouw, K. Winkler, N. Hartmann, and P. Vöhringer. Ultrafast proton-transfer and coherent wavepacket motion of electronically excited 1,8-dihydroxyanthraquinone in liquid benzyl alcohol solution. Zeitschrift für Phys. Chem., 214:1367-1381, 2000.

[47] S.Y. Arzhantsev, S. Takeuchi, and T. Tahara. Ultrafast excited-state proton transfer dynamics of 1,8-dihydroxyanthraquinone (chrysazin) studied by femtosecond time resolved fluorescence spectroscopy. Chem.Phys.Lett., 330:83-90, 2000. 
[48] S.I. Nagaoka and U. Nagashima. Effects of node of wave function upon excited-state intramolecular proton transfer of hydroxyanthraquinones and aminoanthraquinones. Chem.Phys., 206:353-362, 1996.

[49] M.A. Kahlow, W. Jarzeba, T.P. DuBruil, and P.F. Barbara. Ultrafast emission spectroscopy in the ultraviolet by time-gated upconversion. Rev.Sci.Instrum., 59(7):1098-1109, 1988.

[50] Michael Bass, editor. Handbook of optics. Mc Graw-Hill,Inc., 1995.

[51] J. Shah. Ultrafast luminescence spectroscopy using sum frequency generation. J.Quantum Electron., 24:276-288, 1988.

[52] W. Kaiser. Ultrashort Laser Pulses and Applications, chapter 3.2.2. Springer Verlag, 1988.

[53] F. Zernike and J.E. Midwinter. Applied Nonlinear Optics, chapter 6. Wiley \& Sons, 1973.

[54] K.D. Mielenz, E.D. Cehelnik, and R.L. McKenzie. Elimination of polarization bias in fluorescence intensity measurements. J.Chem.Phys., 64:370-374, 1976.

[55] ed. C. Rullière, editor. Femtosecond laser pulses: principles and experiment. Springer, 1998.

[56] M.J. Feldstein, P. Vöhringer, and N.F. Scherer. Rapid-scan pump-probe spectroscopy with high time and wave-number resolution: optical-kerr-effect measurements of neat liquids. J.Opt.Soc.Am.B, 12:1500-1510, 1995.

[57] K. Winkler, J. Lindner, H. Bürsing, and P. Vöhringer. Ultrafast ramaninduced kerr-effect of water. single molecule versus collective motions. Chem.Phys., 113:4674-4682, 2000.

[58] S. Pommeret, R. Naskrecki, J.C. Mialocq, K. Ekvall, C. Dhollande, L.E. Berg, and P. van der Meulen. Cross phase modulation artifact in liquid phase transient absorption spectroscopy. J.Appl.Phys., 87:2340-2352, 2000.

[59] D.W. Tufts and R. Kumaresan. Singular value decomposition and improved frequency estimation using linear prediction. IEEE Trans. Acoust.Speech Signal Process., 30:671-675, 1982.

[60] H. Barkhuijsen, R. de Beer, W.M.M. Bovée, and D. van Ormondt. Retrieval of frequencies, amplitudes, damping factors and phases from time domain signals using a linear least-squares procedure. J.Magn.Reson., 61:465-481, 1985. 
[61] T. Elsaesser and W. Kaiser. Vibrational and vibronic relaxation of large polyatomic molecules in liquids. Annu.Rev.Phys.Chem., 42:83-107, 1991.

[62] D.R. Lide, editor. Handbook of chemistry and physics. CRC Press, 2002.

[63] W.T. Pollard, S.Y. Lee, and R.A. Mathies. Wave packet theory of dynamic absorption spectra and pump-probe experiments. J.Chem.Phys., 92:40124029, 1990.

[64] D. Madsen, J. Stenger, J. Dreyer, E.T.J. Nibbering, P. Hamm, and T. Elsaesser. Coherent vibrational ground-state dynamics of an intramolecular hydrogen bond. Chem.Phys.Lett., 341:56-62, 2001.

[65] J. Stenger, D. Madsen, J. Dreyer, E.T.H. Nibbering, P. Hamm, and T. Elsaesser. Ultrafast dynamics of hydrogen bonds in the liquid phase: vibrational quantum beats and dephasing. In A. Douhal and J. Santamaria, editors, Femtochemistry and Femtobiology, pages 168-185. World Scientific Publishing, 2002.

[66] D. Madsen, J. Stenger, J. Dreyer, P. Hamm, E.T.J. Nibbering, and T. Elsaesser. Femtosecond mid-infrared pump-probe study of wave packet motion in a medium strong intramolecular hydrogen bond. Bull.Chem.Soc.Jpn., 75:909-917, 2002.

[67] C. Chudoba, E. Riedle, M. Pfeiffer, and T. Elsaesser. Vibrational coherence in ultrafast excited state proton transfer. Chem.Phys.Lett., 263:622-628, 1996.

[68] M. Pfeiffer, A. Lau, K. Lenz, and T. Elsaesser. Anharmonicity effects in the resonance raman spectra of heterocyclic aromatic molecules showing photoinduced intramolecular proton transfer. Chem.Phys.Lett., 268:258-264, 1997. 


\section{Danksagung}

Mein Dank gilt Herrn Prof. Dr. Jürgen Troe, da er mir durch die Übernahme des Referats diese Arbeit ermöglicht hat. Ebenso möchte ich Herrn Prof. Dr. M. Buback meinen Dank dafür aussprechen, dass er das Korreferat übernommen hat.

Für die Themenstellung, den Einblick in die faszinierende Forschungswelt des Max-Planck-Instituts und die lebendigen Diskussionen danke ich Herrn Priv.Doz. Dr. Peter Vöhringer.

Herrn Dr. Jörg Lindner danke ich für die wertvollen Ratschläge eines erfahrenen Wissenschaftlers und das Korrekturlesen dieser Arbeit.

Ganz besonders möchte ich auch Herrn Jay Jethwa danken, für seine unverzichtbare Unterstützung in Sachen Elektronik und Lasersystem, dafür dass er immer wusste, welche hilfreichen Schätze in unseren Schränken und Speicherräumen auf ihren Einsatz warteten und in seiner freundlich, fröhlichen Gelassenheit sehr zu einem guten und entspannten Arbeitsklima beigetragen hat.

Mein besonderer Dank gilt auch Donald Ouw, der die Deuterierung des untersuchten Moleküls vorgenommen hat und mir sehr das Leben mit den "schrecklichen" Chemikalien erleichtert hat. Auch für seine guten Ideen und ihre Realisierung im Hinblick auf die kleinen nützlichen "Tools", die das Leben eines Experimentalisten soviel angenehmer machen.

Herrn Bernhard Reuse möchte ich danken, dass er mir durch die gute Einführung in die Recherche-Systeme der Otto-Hahn-Bibliothek zu Beginn meiner Arbeit eine große Quelle wissenschaftlicher Ideen aufgetan hat.

Weiterhin danke ich allen Mitarbeitern der Optik-Werkstatt unter der Leitung von Herrn Wolfgang Sauermann und der Feinmechanik unter der Leitung von Herrn Manfred Schmidt, deren schnelle und gute Arbeit sehr zum Gelingen der Experimente mit beigetragen hat.

Ganz besonders möchte ich auch Andreas Breidenassel danken, meinem lieben Gemahl, der mir immer seelischen Beistand gegeben hat, wenn die Dinge einmal nicht ganz so liefen wie sie sollten und besonders für das Marathon-Korrekturlesen am Ende dieser Arbeit.

Nicht zuletzt danke ich meiner lieben Mutter dafür, dass sie von Zeit zu Zeit bei mir vorbeigekommen ist, um mich von den kleinen Lasten des Alltags zu befreien und in jeglicher Hinsicht immer für mich da war. 



\section{Lebenslauf}

Am 31.08.1973 wurde ich, Nicole Breidenassel, geb. Hartmann, als Tochter von Peter Hartmann und Gerlinde Hartmann, geb. Kotzian in Hildesheim geboren. Seit dem 29. Juni 2002 bin ich mit Andreas Breidenassel verheiratet. Nach dem Besuch der Grundschule Hohnsen in Hildesheim von 1980 bis 1984 wechselte ich auf das Gymnasium Marienschule in Hildesheim und erwarb dort im Mai 1993 das Zeugnis der allgemeinen Hochschulreife. Zum Wintersemester 1993 immatrikulierte ich mich an der Ruprecht-Karls-Universität in Heidelberg für den Diplomstudiengang Physik. Nach dem Vordiplom im Oktober 1995 besuchte ich im Rahmen eines DAAD-Stipendiums von August bis Dezember 1996 die University of Kentucky in den USA. Im Januar 1999 begann ich unter der offiziellen Betreuung durch Herrn Prof. Dr. Siegfried Hunklinger am Kirchhoff-Institut der Universität Heidelberg meine Diplomarbeit, die ich bei Leica Microsystems in Heidelberg durchgeführt habe. Die Thematik mit der ich mich beschäftigte, war die Entwicklung eines frequenzselektiven, akustooptischen Strahlteilers für den Einsatz in einem konfokalen Fluoreszenzmikroskop. Die Ergebnisse dieser Arbeit führten zur Anmeldung des Patentes "Optische Anordnung für ein Laser-Scanning Mikroskop" als Miterfinderin. Im März 2000 erwarb ich das Diplom der Physik der Universität Heidelberg. Seit April 2000 bin ich am Max-Planck-Institut für biophysikalische Chemie in der Arbeitsgruppe "Biomolekulare und chemische Dynamik" unter Leitung von Herrn Dr. Peter Vöhringer als Doktorandin angestellt. Die offizielle Betreuung der Promotion wurde von Herrn Prof. Dr. Jürgen Troe vom Institut für physikalische Chemie der Universität Göttingen übernommen. In dieser Zeit beschäftigte ich mich mit der Femtosekunden-Spektroskopie zur Protontransfer-Dynamik im elektronisch angeregten Zustand von 1.8-DHAQ. Die Ergebnisse dieser Untersuchungen sind in dieser Arbeit zusammengestellt. 